2014

\title{
A search for a heavy Majorana neutrino and a radiation damage simulation for the HF detector
}

James William Wetzel

University of Iowa

Copyright 2014 James William Wetzel

This dissertation is available at Iowa Research Online: http://ir.uiowa.edu/etd/1417

\section{Recommended Citation}

Wetzel, James William. "A search for a heavy Majorana neutrino and a radiation damage simulation for the HF detector." $\mathrm{PhD}$ (Doctor of Philosophy) thesis, University of Iowa, 2014.

http://ir.uiowa.edu/etd/1417.

Follow this and additional works at: http://ir.uiowa.edu/etd

Part of the Physics Commons 


\title{
A SEARCH FOR A HEAVY MAJORANA NEUTRINO AND A RADIATION DAMAGE SIMULATION FOR THE HF DETECTOR
}

\author{
by \\ James William Wetzel
}

A thesis submitted in partial fulfillment of the requirements for the Doctor of Philosophy degree in Physics

in the Graduate College of

The University of Iowa

August 2014

Thesis Supervisor: Associate Professor Jane Nachtman 


\section{Graduate College \\ The University of Iowa \\ Iowa City, Iowa}

\section{CERTIFICATE OF APPROVAL}

\section{PH.D. THESIS}

This is to certify that the Ph.D. thesis of

\section{James William Wetzel}

has been approved by the Examining Committee for the thesis requirement for the Doctor of Philosophy degree in Physics at the August 2014 graduation.

Thesis Committee:

Jane Nachtman, Thesis Supervisor

Yasar Onel

Vincent Rodgers

Robert Mutel

John Ellison 
To my parents, for being the incredible duo they are. 
Beginning today, treat everyone you meet as if they were going to be dead by midnight. Extend to them all the care, kindness, and understanding you can muster, and do it with no thought of any reward. Your life will never be the same again.

Og Mandino 


\section{ACKNOWLEDGEMENTS}

I owe a lifetime of gratitude to Professor Yasar Onel for his support, mentorship, and his inspiration to push myself to be a better person and a better physicist every day. I, and many others, would not be here if it were not for him.

I also must also acknowledge the friendship, mentorship, kindness, and support Professor Ugur Akgun has shown me. He is an inspiration to me as an instructor, a researcher, a husband, and a father, and if it weren't for his support, I would never have dreamed of embarking on this journey.

I have to thank Professor Vincent Rodgers for accepting me, an experimental physicist, into his courses on general relativity and quantum field theory. These courses gave me profound respect for the combination of mathematics and those with the creativity necessary to see the 'real' world. Time spent in those classes became a major source of inspiration for me to understand the processes of the universe more deeply, leaving an impression that will stay with me for my lifetime.

Professor Robert Mutel has had a unique and important impact on me, as a scientist, as an instructor, and as a family man during my time at Iowa. I must thank him for showing me the world of the telescope, and allowing me the opportunity to help shape the astronomy lab courses. His vision for thinking up crazy ideas and his ability to make them happen has had a deep impact, pun intended, on me.

I have to especially thank my colleagues on the search for a Majorana neutrino. Professor John Ellison has been an exceptional mentor to me, helping me along, (and 
being very patient), while I learned the intricacies of performing a CMS analysis. Dr. Ferdinando Giordano, whose countless FaceTimes, iMessages, and emails during almost all hours of these past days and nights are a testament to his dedication, expertise, and kindness. Though we have yet to meet face-to-face, we now have a bond that only CERN can create between people.

I am very thankful to Professor Jane Nachtman for chairing my committee, and accepting me as her student. I am forever grateful for the amazing opportunity she has given me.

I offer an infinite number of thank-yous to the University of Iowa's Physics Department staff. Their professionalism, expertise, and knowledge has been extremely helpful.

Beyond that, I want to express the highest gratitude to those who have set the standards for the rest of us to meet and to exceed; they are the people who most inspire me.

Just as important are my family and friends for being there to catch my fall, to get me back up, and to push me to try again; for expecting me to accept challenges, and encouraging me to endlessly try until I succeed. It has not been easy.

Lastly, to my dearest Shelly: I haven’t stopped smiling since I met you. 


\begin{abstract}
A search for heavy Majorana neutrinos is performed using an event signature defined by two same-sign muons accompanied by two jets. This search is an extension of previous searches, (L3, DELPHI, CMS, ATLAS), using $19.7 \mathrm{fb}^{-1}$ of data from the 2012 Large Hadron Collider experimental run collected by the Compact Muon Solenoid experiment. A mass window of $40-500 \mathrm{GeV} / c^{2}$ is explored. No excess events above Standard Model backgrounds is observed, and limits are set on the mixing element squared, $\left|V_{\mu N}\right|^{2}$, as a function of Majorana neutrino mass.

The Hadronic Forward (HF) Detector's performance will degrade as a function of the number of particles delivered to the detector over time, a quantity referred to as integrated luminosity and measured in inverse femtobarns $\left(\mathrm{fb}^{-1}\right)$. In order to better plan detector upgrades, the CMS Forward Calorimetry Task Force (FCAL) group and the CMS Hadronic Calorimeter (HCAL) group have requested that radiation damage be simulated and the subsequent performance of the HF subdetector be studied. The simulation was implemented into both the CMS FastSim and CMS FullSim simulation packages. Standard calorimetry performance metrics were computed and are reported. The HF detector can expect to perform well through the planned delivery of $3000 \mathrm{fb}^{-1}$.
\end{abstract}




\title{
TABLE OF CONTENTS
}

LIST OF TABLES . . . . . . . . . . . . . . . . . .

LIST OF FIGURES . . . . . . . . . . . . . . . . . . xii

\author{
CHAPTER
}

1 THE STANDARD MODEL OF PARTICLE PHYSICS . . . . . . . . 1

1.1 Survey of the Standard Model . . . . . . . . . . . . . . . 1

1.2 History . . . . . . . . . . . . . . . . . . . . . 4

1.2.1 The Foundation . . . . . . . . . . . . . . . 4

1.2 .2 The Photon . . . . . . . . . . . . . . 5

1.2.3 Meson: The First Exotic Particle . . . . . . . . . . . . . 6

1.2.4 Anti-matter . . . . . . . . . . . . . . . 7

1.2.5 Neutrinos and Lepton Number . . . . . . . . . . . . 7

1.2 .6 The Particle Zoo . . . . . . . . . . . . . . . . . . . 11

1.2.7 Strange Particles and the Eightfold Way . . . . . . . . . . 11

1.2 .8 Quarks . . . . . . . . . . . . . . . . . . . 14

1.2 .9 Color Charge . . . . . . . . . . . . . . . . . . . . . . 15

1.2 .10 GWS and the Vector Bosons . . . . . . . . . . . 16

1.2 .11 The Higgs Boson . . . . . . . . . . . . . . . . . . . . . 16

1.2 .12 The Present . . . . . . . . . . . . . . . . . . 17

1.2 .13 The Future . . . . . . . . . . . . . . . . . . . . 18

1.3 Particle Physics Pertinent to this Analysis . . . . . . . . . . 20

1.3.1 Cross Section in Detector-Based Particle Physics . . . . . 20

1.3.2 An Example . . . . . . . . . . . . . . . . . . . . . 21

1.3.3 Energy Resolution of a Calorimeter . . . . . . . . . 22

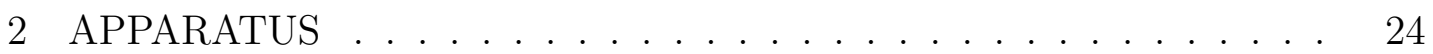

2.1 Introduction . . . . . . . . . . . . . . . . . . . . 24

2.2 The Large Hadron Collider . . . . . . . . . . . . . . . . 25

2.2 .1 Principles of Design . . . . . . . . . . . . . . . . 26

2.2 .2 Proton Injection . . . . . . . . . . . . . . . . 26

2.2 .3 Beam Control . . . . . . . . . . . . . . . . . 30

2.3 The Compact Muon Solenoid Detector . . . . . . . . . . . . 33

2.3 .1 Principles of Design . . . . . . . . . . . . . . . 34

2.3.2 Inner Tracker . . . . . . . . . . . . . . . . . . . 37

2.3.3 Electromagnetic Calorimeter . . . . . . . . . . . . . 40

2.3.4 Hadronic Calorimeter . . . . . . . . . . . . . . . . 43 
2.3 .5 Muon System . . . . . . . . . . . . . . . . . . . . . . . . . . . . . . . . 45

2.3 .6 Trigger . . . . . . . . . . . . . . . . . . . 47

2.3 .7 CMS Software . . . . . . . . . . . . . . . . 47

2.3.8 CMSSW and CMS Simulation . . . . . . . . . . 50

2.4 Particle Identification $\ldots \ldots \ldots \ldots$

2.4 .1 Muon Reconstruction . . . . . . . . . . . . . . . . 51

2.4 .2 Jet Reconstruction . . . . . . . . . . . . . . . . . . 52

2.4.3 Jet Energy and Resolution Scales . . . . . . . . . . . . . 53

2.4 .4 b-Tag Jets . . . . . . . . . . . . . . . . . . . . 54

2.4 .5 Particle Flow . . . . . . . . . . . . . . . . 54

3 A SEARCH FOR A HEAVY MAJORANA NEUTRINO . . . . . . . 56

3.1 Introduction $\ldots \ldots \ldots \ldots \ldots \ldots \ldots \ldots \ldots$

3.2 Monte Carlo Event Generation . . . . . . . . . . . . . . . . . 58

3.2 .1 Signal Event Generation . . . . . . . . . . . . . 60

3.2 .2 Simulation . . . . . . . . . . . . . . . . . . 61

3.2.3 Standard Model Monte Carlo Samples . . . . . . . . . . . 62

3.3 LHC Collision Datasets . . . . . . . . . . . . . . . . . . . . 62

3.3 .12012 Collision Data . . . . . . . . . . . . . . . . . 65

3.4 Event Selection . . . . . . . . . . . . . . . . . . . . . . . 66

3.4 .1 Trigger . . . . . . . . . . . . . . . . . 67

3.4 .2 Primary Vertex Selection . . . . . . . . . . . . 67

3.4 .3 Muon Selection . . . . . . . . . . . . . . . . . . . 68

3.4 .4 Jet Selection . . . . . . . . . . . . . . . . . . 70

3.4.5 Event Preselection . . . . . . . . . . . . . . . . 71

3.4 .6 Full Selection . . . . . . . . . . . . . . . . . . . 74

3.4.7 Final Selection Optimization . . . . . . . . . . 76

3.5 Selection Efficiency . . . . . . . . . . . . . . . . 77

3.5 .1 Trigger Efficiency . . . . . . . . . . . . . . . . 77

3.5.2 Offline Selection Efficiency _.. . . . . . . . . . . . 78

3.5.3 Tag and Probe Study . . . . . . . . . . . . . . . . 81

3.6 Background Estimation . . . . . . . . . . . . . . . . . 83

3.6.1 The Fake Rate Method . . . . . . . . . . . . . . . . 88

3.6.2 Fake Rate Measurement in Data . . . . . . . . . . . . 91

3.6.3 Validation of the Fake Rate Method . . . . . . . . . . . 95

3.7 Selection Results . . . . . . . . . . . . . . . . . . . . . . 100

3.7.1 Low Mass Selection Results . . . . . . . . . . . . . . . 100

3.7 .2 High Mass Selection Results . . . . . . . . . . . . . 102

3.8 Systematic Uncertainties . . . . . . . . . . . . . . . . . . . 102

3.9 Interpretation of Results . . . . . . . . . . . . . . . 106

3.10 Future Prospects . . . . . . . . . . . . . . . . . . . 107

4 A RADIATION DAMAGE SIMULATION FOR THE HF DETECTOR 111 
4.1 Introduction to $\mathrm{HF} \quad \ldots \ldots \ldots \ldots$

4.2 Radiation Damage in HF . . . . . . . . . . . . . . . . . . . 114

4.3 Modeling Radiation Damage in HF . . . . . . . . . . . . . . . . . 114

4.3.1 Implementation . . . . . . . . . . . . . . . . . . . . . 114

4.4 Results . . . . . . . . . . . . . . . . 116

4.4.1 Correction Factors . . . . . . . . . . . . . . 116

4.4 .2 Jets in $\mathrm{HF}$. . . . . . . . . . . . . . . . . . . . . . . . 117

4.5 Conclusion . . . . . . . . . . . . . . . . . . 117

4.6 Future prospects . . . . . . . . . . . . . . . . . . 121

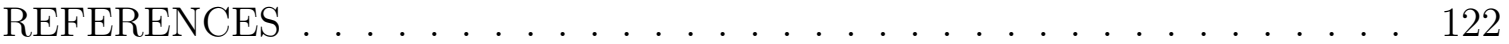




\section{LIST OF TABLES}

Table

1.1 Summary of Standard Model Particles . . . . . . . . . . . . . . . . . . . 1

1.2 Particle Families . . . . . . . . . . . . . . . . . . 2

1.3 Summary of Standard Model Forces . . . . . . . . . . . . . . . . . . 3

1.4 Summary of Standard Model Leptons. . . . . . . . . . . . . . . . . . . . 4

2.1 Summary of the LHC design characteristics. . . . . . . . . . . . . . . . . 29

3.1 Summary of Standard Model Monte Carlo datasets used. . . . . . . . . . 63

3.2 Majorana neutrino signal MC samples generated with $\left|V_{\mu N}\right|^{2}=1 . \quad$. . . $\quad 64$

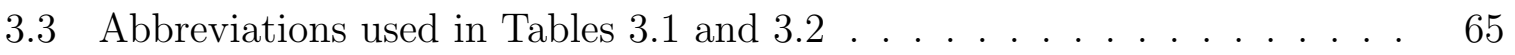

3.4 Summary of 2012 datasets used. . . . . . . . . . . . . . . . . . . . 66

3.5 Muon selection and individual cut efficiencies for events in Signal MC passing the selection cuts listed in Sections 3.4.3 - 3.4.6. . . . . . . . . . 79

3.6 Event selection efficiencies for the events in Signal MC passing the selection cuts listed in Sections 3.4.3-3.4.6. . . . . . . . . . . . . 80

3.7 Tag and Probe Muon ID efficiencies for MC and data, $\eta$ region 1 and 2. . 86

3.8 Tag and Probe Muon ID efficiencies for MC and data, $\eta$ region 3 and 4. . 87

3.9 Muon fake rates obtained from data in bins of $|\eta|$ and $\mathrm{p}_{T} . \quad \ldots . . . . \quad 95$

3.10 Predicted (\#FR) and observed (\#MC) fake backgrounds in $t \bar{t}, W+$ jets, and QCD Monte Carlo samples for events passing preselection. . . . . . . 100

3.11 Observed event yields and estimated backgrounds with statistical and systematic uncertainties for the low mass selection. . . . . . . . . . . . . 101

3.12 Results of the cut optimization for the low mass Majorana neutrino region. 102 
3.13 Observed event yields and estimated backgrounds with statistical and systematic uncertainties for the high mass selection. . . . . . . . . . . 103

3.14 Results of the cut optimization for the 'high mass' region. . . . . . . . . 103

3.15 Summary of systematic uncertainties. . . . . . . . . . . . . . 105

4.1 Efficiencies for varying jet $\mathrm{p}_{T}$ and integrated luminosity for jets from single down quarks in the HF detector. . . . . . . . . . . . . . . . 119

$4.2100 \mathrm{GeV}$ jet efficiency vs. $\eta \ldots \ldots \ldots$ 


\section{LIST OF FIGURES}

Figure

1.1 The discovery of the positron in a bubble chamber. . . . . . . . . . . 8

1.2 The discovery of the first strange particle, the neutral Kaon, $K^{0} \ldots \ldots$. . 12

1.3 The Brookhaven Cosmotron, the first of the modern particle accelerators, and the first to reach the GeV scale. . . . . . . . . . . . . . . . 13

1.4 The Baryon Octet . . . . . . . . . . . . . . . . . . 19

1.5 The Meson Octet . . . . . . . . . . . . . . . . . . . 20

1.6 HF detector response to $150 \mathrm{GeV} / c$ jets. . . . . . . . . . . . . 23

1.7 A simple Gaussian fit to $150 \mathrm{GeV} / c$ pions in HF. . . . . . . . . . . . . . 23

2.1 Map of the CERN site . . . . . . . . . . . . . . . . . . 25

2.2 LHC Dipole Magnet Cross Section. . . . . . . . . . . . . . . . . . . . . . 27

2.3 LHC Dipole Magnet B-Field. . . . . . . . . . . . . . . . . . . . . . . 27

2.4 Inside the LHC Accelerator Tunnel. . . . . . . . . . . . . . . . . . . . . 28

2.5 The CERN Accelerator Complex. . . . . . . . . . . . . . . . 28

2.6 Longitudinal phase diagram for an accelerating RF system . . . . . . . . 31

2.7 Tidal deformation of the Earth's surface (left) and resulting LHC tunnel deformation (right). . . . . . . . . . . . . . . . . . . 32

2.8 The CMS detector in the experimental cavern. . . . . . . . . . . . . . 33

2.9 Cutaway isometric view of the CMS detector . . . . . . . . . . . 36

2.10 Particle flow in the CMS Detector. . . . . . . . . . . . 36

2.11 The CMS Tracking System viewed along the beam line. . . . . . . . . . 37 
2.12 The CMS Tracking system schematic. . . . . . . . . . . . . . . . 39

2.13 The silicon strip inner barrel of the CMS tracking system. . . . . . . . . 39

2.14 Italian physicist Francesca Cavallari beneath the ECAL Barrel. . . . . . 40

2.15 ECAL cutaway showing endcap (EE) and barrel (EB) subdetectors. . . . 41

2.16 ECAL $\mathrm{PbWO}_{4}$ crystal shower simulation of a $24 \mathrm{GeV} e^{-} \ldots \ldots$. . . . . 42

2.17 Russian Navy shells used to create the brass tiles of HCAL. . . . . . . . 43

2.18 A segment of the CMS muon system. . . . . . . . . . . . . . 45

2.19 Longitudinal quadrant view of the CMS muon system. . . . . . . . . . 46

2.20 Simulated strength (left) and field lines (right) of the B-field in CMS. . . 46

3.1 A Feynman diagram for resonance production of a Majorana Neutrino $(\mathrm{N})$ in hadron colliders. . . . . . . . . . . . . . . . . . . 59

3.2 Cross sections for resonance production of a Majorana neutrino as a function of Majorana neutrino mass. . . . . . . . . . . . . . . . 59

3.3 The invariant mass of the two selected muons at preselection. . . . . . . 72

3.4 The invariant mass of the two selected muons and two selected jets at preselection. ....................... 73

3.5 Example Z Boson mass peak from $Z \rightarrow \mu \mu$ in collision data. . . . . . . . 84

3.6 Example Z Boson mass peak from $Z \rightarrow \mu \mu$ in Monte Carlo data. . . . . . 84

3.7 Muon Efficiencies using the Tag and Probe method for $\eta 0-1.2 \ldots . . . \quad 85$

3.8 Muon Efficiencies using the Tag and Probe method for $\eta 1.2-2.4 \ldots 5$

3.9 The $\mathrm{p}_{T}$ spectrum of jets tagged as a fake object in MC. . . . . . . . . . 92

3.10 The $m(l l)$ distribution of the events in the fake-rate control region. . . . 93

3.11 The transverse mass $\left(M_{T}\right)$ distribution of the events in the fake-rate control region. . . . . . . . . . . . . . . . . 94 
$3.12 p_{T}$ distributions for the tight muons (top) and loose muons (bottom) used in the fake rate calculation. . . . . . . . . . . . . . 96

$3.13 \eta$ distributions for the tight muons (top) and loose muons (bottom) used in the fake rate calculation. . . . . . . . . . . . . . . . . . 97

3.14 Muon fake rates obtained from data as a function of $\eta$ and $\mathrm{p}_{T} \ldots \ldots$. . . 98

3.15 The invariant four-mass $(\mu \mu j j)$, for events passing the low mass selection. 101

3.16 Exclusion region of the HMN mixing element vs. mass. . . . . . . . . . . 108

3.17 Zoom of Figure 3.16 at low mass. . . . . . . . . . . . . . . 109

3.18 HF Lowering. . . . . . . . . . . . . . . . . . . . . . . . . . . 110

4.1 HF Wedges. . . . . . . . . . . . . . . . . . . . 112

4.2 HF Tower Geometry. . . . . . . . . . . . . . . . . . . . . 113

$4.3 \mathrm{HF}$ dose map cells. . . . . . . . . . . . . . . . . . . . . . . . . . . . 115

4.4 A sample of HF recalibration parameterizations. . . . . . . . . . . . . . . 118

4.5 Energy resolution of the HF detector. . . . . . . . . . . . . . . . . . 120 


\section{CHAPTER 1 \\ THE STANDARD MODEL OF PARTICLE PHYSICS}

What follows is a very brief and decidedly experiment-centric introduction and history to modern particle physics. The goal is to give the reader some context for the experiments described in this thesis. One may consult the references for a more fulfilling treatment. [1, 2, 3]

\subsection{Survey of the Standard Model}

I have chosen to start with a summary of the Standard Model, followed with a brief history of its past, in the hopes of leaving the reader with better context for the history as it is described.

The Standard Model of particle physics describes the forces and interactions - except gravity - of all known matter in our universe. It is a field theory at its heart, and has successfully predicted the existence of many fundamental particles with incredible precision. The current Standard Model contains 17 fundamental particles, (61 in all their various permutations), listed in Table 1.1.

These particles can be grouped into two families: bosons and fermions, see

Table 1.1: Summary of Standard Model Particles

\begin{tabular}{ll}
\hline Classification & Particle \\
\hline Quarks & $u, d, s, c, b, t$ \\
Leptons & $e, \nu_{e}, \mu, \nu_{\mu}, \tau, \nu_{\tau}$ \\
Force Carriers & $W, Z, \gamma, g, H$ \\
\hline
\end{tabular}


Table 1.2: Particle Families

\begin{tabular}{lll}
\hline Family & Particle & Spin \\
\hline Fermions & $u, d, s, c, b, t$ & $1 / 2$ Integer \\
& $e, \nu_{e}, \mu, \nu_{\mu}, \tau, \nu_{\tau}$ & \\
Bosons & $W, Z, \gamma, g, H$ & Integer \\
\hline
\end{tabular}

Table 1.2 .

Fermions, the spin halves, obey both Fermi statistics and the famous Pauli Exclusion Principle, which disallows two fermions from occupying the same space at the same time, (in other words having the same quantum numbers). Bosons obey Bose-Einstein statistics, where two or more identical particles may occupy the same space, meaning they can have identical quantum numbers. This difference between fermions and bosons has to do with a particle's property called $\operatorname{spin}^{1}$.

Fermions can be grouped further into two classifications: quarks and leptons. Quarks obey a process called quark confinement, and cannot exist independently; they are only found in bound states. (For example, a neutron is a bound state of one up and two down quarks ${ }^{2}$.) Leptons, however, are free to roam on their own. In fact,

${ }^{1}$ Spin is sort of like a particle's version of the angular momentum of a spinning basketballsort of. A rotating, charged object, like the earth, creates a magnetic field. Similarly, an electron is charged and has a measureable magnetic field. From this we can assign a sort of angular momentum - spin - to the electron, (although it isn't literally spinning like a basketball, and a particle does not require classical charge to have a spin, nor does it have to generate a magnetic field if it has spin). Spin simply explains why an electron - or any charged particle - has its own magnetic field, and is used to describe how two identical particles will interact with each other. We await the physicists of the future to discover why a particle has spin.

${ }^{2}$ Technically, a neutron is composed of a sea of quarks, anti-quarks, and gluons, but it works out to have $n$ up quarks and $n+1$ down quarks. 
Table 1.3: Summary of Standard Model Forces

\begin{tabular}{llll}
\hline Force & Theory & Mediator & Example Interactions \\
\hline Strong & QCD & Gluons & Bound quarks \\
Weak & Electroweak & W \& Z Bosons & Beta Decay \\
Electromagnetic & QED & Photons & Magnetism \\
\hline
\end{tabular}

every second one hundred billion leptons (neutrinos) fly right through your thumb! The other family, the bosons, the integer spins, are the force carriers. They are responsible for mediating the fundamental forces and interactions between particles; summarized next.

The Standard Model describes three fundamental ways particles can interact, listed in Table 1.3. For example, the quarks that make up the neutron are bound together by the strong force, mediated by gluons. A magnetic picture frame sticks to your refrigerator because of the electromagnetic force, mediated by virtual photons. The weak interaction allows for the transmutation of a quark of one flavor to a quark of another flavor. This allows a neutron to decay to a proton, for example, by the transmutation of a down quark to an up quark by emitting the charged boson, $\mathrm{W}^{-}$, which then decays to an electron, $e$, and an electron neutrino, $\nu_{e}$, perhaps recognized as the famous beta decay. The weak force and the electromagnetic force unify at high energy to become the electroweak force.

In technical language, the Standard Model is a gauge theory that can be neatly written as:

$$
S U(3)_{Q C D} \times\left(S U_{L}(2) \times U(1)\right)_{G W S}
$$


Table 1.4: Summary of Standard Model Leptons.

\begin{tabular}{lcccc}
\hline Lepton & Lepton \# & Electron \# & Muon \# & Tau \# \\
\hline$e^{-}$ & 1 & 1 & 0 & 0 \\
$\nu_{e}$ & 1 & 1 & 0 & 0 \\
$\mu^{-}$ & 1 & 0 & 1 & 0 \\
$\nu_{\mu}$ & 1 & 0 & 1 & 0 \\
$\tau^{-}$ & 1 & 0 & 0 & 1 \\
$\nu_{\tau}$ & 1 & 0 & 0 & 1
\end{tabular}

\begin{tabular}{lcccc}
\hline Antilepton & Lepton \# & Electron \# & Muon \# & Tau \# \\
\hline$e^{+}$ & -1 & -1 & 0 & 0 \\
$\overline{\nu_{e}}$ & -1 & -1 & 0 & 0 \\
$\mu^{+}$ & -1 & 0 & -1 & 0 \\
$\overline{\nu_{\mu}}$ & -1 & 0 & -1 & 0 \\
$\tau^{+}$ & -1 & 0 & 0 & -1 \\
$\overline{\nu_{\tau}}$ & -1 & 0 & 0 & -1 \\
& & & & \\
\hline
\end{tabular}

\subsection{History}

\subsubsection{The Foundation}

By most accounts, the era of modern particle physics began in 1897 with the discovery of the electron by J. J. Thomson. From there, Ernest Rutherford's discovery of the atom's nucleus in 1911 led to a description of the hydrogen atom in 1914 by Niels Bohr consisting of a single electron orbiting the atom's nucleus. Bohr's calculation of hydrogen's spectrum agreed very well with experiment, but a problem occurred when his model was scaled to heavier atoms - his theory broke down.

This crisis of sorts was solved more than 15 years later when James Chadwick (a student of Ernest Rutherford) discovered the neutron.

At this point, in 1932, the atomic world consisted only of the electron, the 
newly-discovered neutron, and the proton, and what a simple world it was.

\subsubsection{The Photon}

Parallel to this effort, Albert Einstein and Max Planck were busy uncovering the secrets of the as-yet-unnamed photon. Planck showed that the light-energy radiated by a hot object, called a 'blackbody', was quantized, or came in discrete bits whose magnitude depended only on the frequency of the radiation ${ }^{3}$. Einstein, in 1905, hypothesized in his Nobel Prize winning paper that it wasn't just in the case of a blackbody that the energy was emitted in bits, but that the electromagnetic field itself was quantized. This result led to the formulation of the well known Photoelectric Effect.

More than 10 years later, in 1916, Robert Andrews Millikan showed that Einstein's photoelectric effect perfectly predicted the observed results of his experiments. Though Einstein's theory was validated, he still faced opposition to his radical idea that light not only behaved like a particle, but in fact was a particle. Finally, 18 years after Einstein first proposed the idea, Arthur Compton conducted the definitive experiment, one which provided strong evidence for the existence of the photon by firing x-rays with known energy, E, into a graphite target and measuring the resulting energy, $E^{\prime}$, as a function of its scattering angle. Light behaved just like a cue ball in the game of pool, knocking into electrons one by one.

\footnotetext{
${ }^{3}$ Planck actually believed his theory was wrong, and insisted that nature didn't work this way. He often remarked that it would take a future physicist to realize his mistake. Of course, it turned out that this revolutionary idea became the cornerstone of modern physics.
} 
Joining the neutrons, protons, and electrons of the atomic world, the photon took its place in early particle physics as an active player with an unknown origin.

\subsubsection{Meson: The First Exotic Particle}

A big question of this time, one that you may (or may not) have asked yourself, is if positive charges repel, what is keeping the protons of the atom's nucleus together? After all, an iron atom has 26 protons tightly packed together, while a gold atom has 79 protons crammed in there! In knowing that it must be a mighty force that keeps these protons so tightly bound, physicists dubbed it the strong force.

In 1935 Hideki Yukawa was the first to create a theory of the strong force, which required the existence of a particle called a 'meson', meaning 'middleweight'. He calculated its mass to be between the electron, (a lepton, meaning 'lightweight') and the proton, (a baryon, meaning 'heavyweight'), hence the name meson. His particle was discovered in 1937 (along with an anomaly) by studying the tracks of cosmic particles left on photographic plates placed high up on mountain ranges. It wasn't until 1947 that the anomaly was explained and it was shown that Yukawa's meson was decaying into another middleweight particle, a particle that was later determined to be a muon. With the discovery of the muon, there were now two mesons in the ranks of middleweight particles. Yukawa's meson was renamed 'pion', and the muon reclassified as a lepton, though many publications during this era continued to refer to the muon as the mu-meson. 


\subsubsection{Anti-matter}

During this foray into cosmic particles and interactions, Paul Dirac was busy working on formulating relativistic quantum mechanics. His derived and so-named Dirac equation, written in 1927, implied the existence of positive and negative energy states. It wasn't until 1940 that Richard Feynman and Ernst Stuckelberg described the negative energy solutions as positive energy states of a new particle. While Dirac's equation indeed predicted the existence of the electron, and matched the properties physicists had already measured, it also predicted the existence of an electron's positively charged twin, the positron. In 1932 Carl Anderson discovered a particle with the same mass as the electron, but opposite charge, just as Dirac's equation predicted; see Figure 1.1. In fact, Dirac's equation predicted the existence of a twin for every particle, what we now call anti-matter: for every particle described by some mass and their quantum numbers, an anti-particle exists with the same mass and opposite-signed quantum numbers.

The world of particles was becoming quite interesting, and quite crowded.

\subsubsection{Neutrinos and Lepton Number}

Around the same time that the neutron was discovered (1932), physicists had been tinkering around with the process of beta decay, which occurs when a neutron is converted into a proton and emits an electron, $\left(n \rightarrow p^{+}+e^{-}\right)$. When considering a two-body decay like this, the energy of the electron should be fixed. However, the experiments came back with startling results: The measured energy of the resulting 


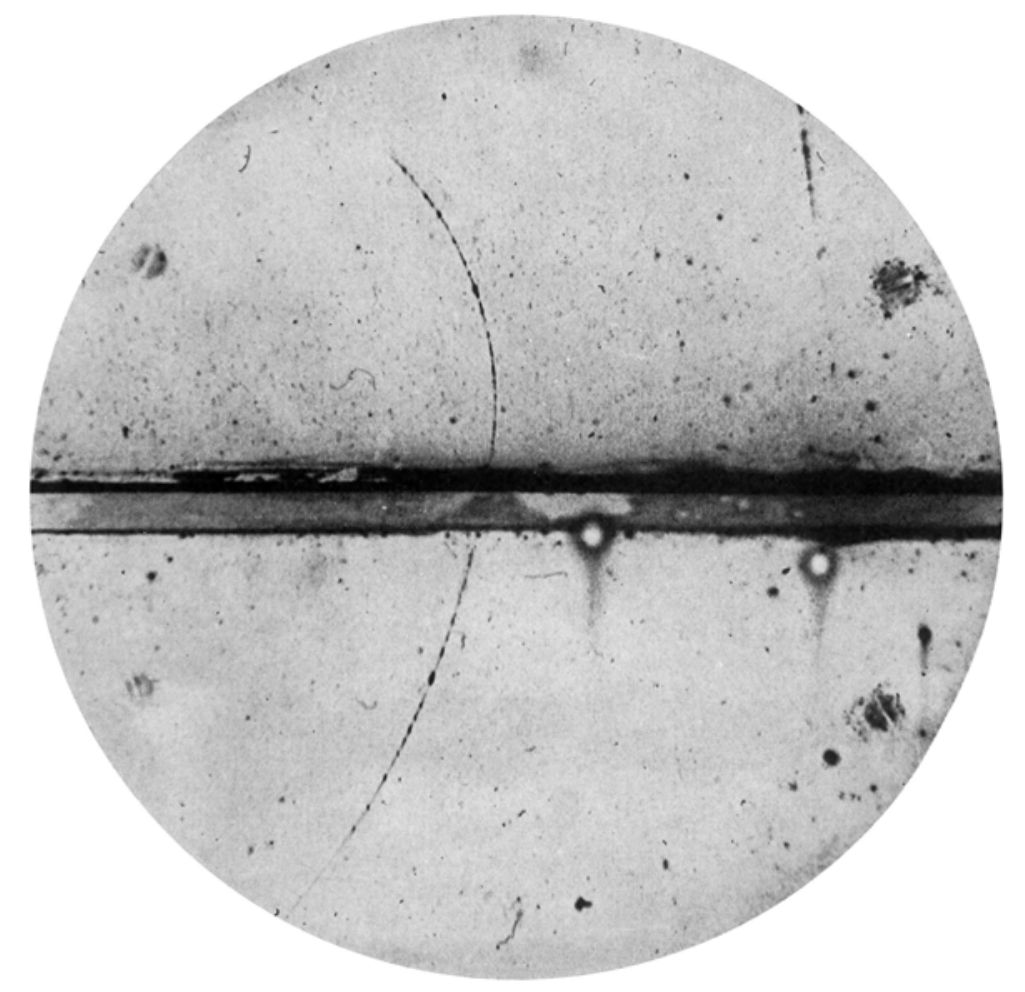

Figure 1.1: The discovery of the positron in a bubble chamber. With a magnetic field pointing into the page, this track could be produced by a negatively charged particle traveling top $\rightarrow$ down, or a positively charged particle traveling bottom $\rightarrow$ up. To differentiate, Carl Anderson placed a lead plate through the center of the chamber, slowing the particle down and tightening the curve of its trajectory. 
electron was a distribution!

Either the law of conservation of energy had to be thrown out, or a new particle had to be introduced. Wolfgang Pauli hypothesized the existence of a third particle in the decay products to share energy with the electron. In 1934 Enrico Fermi produced his theory of beta decay, which proved to be extremely successful and ended up laying the foundation for all weak force physics. In Fermi's formulation, beta decay can be written as $\left(n \rightarrow p^{+}+e^{-}+\bar{\nu}_{e}\right)$, and includes the particle he named the neutrino, meaning 'little neutral one'. Despite his theory's successful predictions, it wasn't until 1953 - almost 20 years later - that the first direct experimental evidence for neutrinos was produced ${ }^{4}$.

Since the neutrino is neutral, an obvious question is "what is the difference between the neutrino and the anti-neutrino'? This question touches on the heart of the analysis in this thesis. E. J. Konopinski and H. M. Mahmoud first proposed the idea of lepton number in 1953 [6]. The first experimental evidence (or lack thereof)

${ }^{4}$ The Nobel Prize winning Cowan-Reines Neutrino experiment was first conducted at the infamous Hanford site, and later moved to the Savannah River nuclear reactor in South Carolina for better shielding from cosmic rays. These experiments were conducted near nuclear reactors due to the extreme flux of neutrinos they generate. (Though at the time, they didn't yet know it was actually anti-neutrinos they were detecting). The Savannah River reactor generated $5 \times 10^{13}$ anti-neutrinos per second per square centimeter; even at this rate, interactions occurred roughly twice per hour. Cowan and Reines set out to prove the existence of the neutrino using the theory of inverse-beta decay. This process, predicted using Fermi's theory, requires an anti-neutrino to interact with a proton via the weak force, $\left(\bar{\nu}_{e}+p^{+} \rightarrow n+e^{+}\right)$, creating a detectable final state. The positron produced immediately annihilates with an electron, producing one flash of light in the detector, and a fraction of a second later the neutron is captured by a cadmium nucleus, producing a second flash of light. It is this double flash that is the mark of a neutrino, and set in stone the theory of the weak force. The original paper describing the Hanford setup and a fantastic summary of these experiments is given by [4] and [5]. 
that the process $\left(\bar{\nu}_{e}+n \rightarrow p^{+}+e^{-}\right)$was forbidden came in 1959 from Raymond Davis, Jr. and Don Harmer. A lepton number of +1 is assigned to the electron, muon $\left(\mu^{-}\right)$, and neutrino. A lepton number of -1 is assigned to the positron (or anti-electron), the muon $\left(\mu^{+}\right)$, and the anti-neutrino. Non-leptons have a lepton number of zero. On both sides of the arrow, the sum of the lepton numbers must be the same. In other words, lepton number must be conserved. The previous interaction can thus be written $(-1+0 \rightarrow 0+1)$, with a total lepton number of -1 on the left side, and +1 on the right side.

Lepton number conservation "explained" why Cowan and Reines could see their interaction, while Davis and Harmer could not observe theirs.

It was only after the discovery of lepton number that we learned the first difference between an anti-neutrino and a neutrino ${ }^{5}$. There are actually two different theoretical treatments used to describe a neutrino: the Dirac neutrino formulation, and the Majorana neutrino formulation. In the Dirac formulation, a neutrino and its anti-particle are distinct from one another. In the Majorana formulation, the neutrino is its own anti-particle.

Two more "rules" were discovered that govern the interaction of leptons: muon number and electron number. Just like lepton number, muon number and electron number must be conserved. This implies that there are different flavors of neutrinos: muon neutrinos, $\nu_{\mu}$, and electron neutrinos, $\nu_{e}$. The Brookhaven Alternating Gradient

\footnotetext{
${ }^{5}$ The anti-neutrino and neutrino also differ in their helicity, where the anti-neutrino is 'right-handed' and the neutrino is 'left-handed'.
} 
Synchrotron provided the first direct evidence of the two different flavors of neutrinos [7], winning Lederman, Schwartz, and Steinberger the Nobel Prize ${ }^{6}$. See Table 1.4 for a summary of the leptons discovered to date.

\subsubsection{The Particle Zoo}

In 1900, the only particle known was the electron. By 1950, the particle world impressively had grown to include the proton, neutron, muon, neutrino, pion, their respective anti-particles, and the photon.

By 1960, the number of particles discovered (and the number of researchers required to discover them) became too many to name. It started with the discovery of strange particles. The first strange particle discovered was called the neutral kaon, $K^{0}$, followed by its charged sibling, the $K^{+}$, and both were placed into the meson family; see Figure 1.2. Three years later, in 1950, another strange particle, a baryon called the $\Lambda$ (lambda), was discovered. In 1952, the $\Xi$ (cascade) was discovered. Also in 1952, the Brookhaven Cosmotron (see Figure 1.3) came online providing the first ability to create and study rare particles in a more controlled environment.

\subsubsection{Strange Particles and the Eightfold Way}

After the commissioning of the Cosmotron, and subsequent accelerators like the 6.2 GeV Bevatron in 1955, the world of the strange particles began to open up,

\footnotetext{
${ }^{6}$ In 1977 Martin Lewis Perl completed the lepton story by discovering the tau lepton, earning him a Nobel Prize. (The tau lepton's discovery immediately implied the existence of a corresponding tau neutrino, but it wasn't until the year 2000 when the Fermilab DONUT collaboration announced its discovery.)
} 


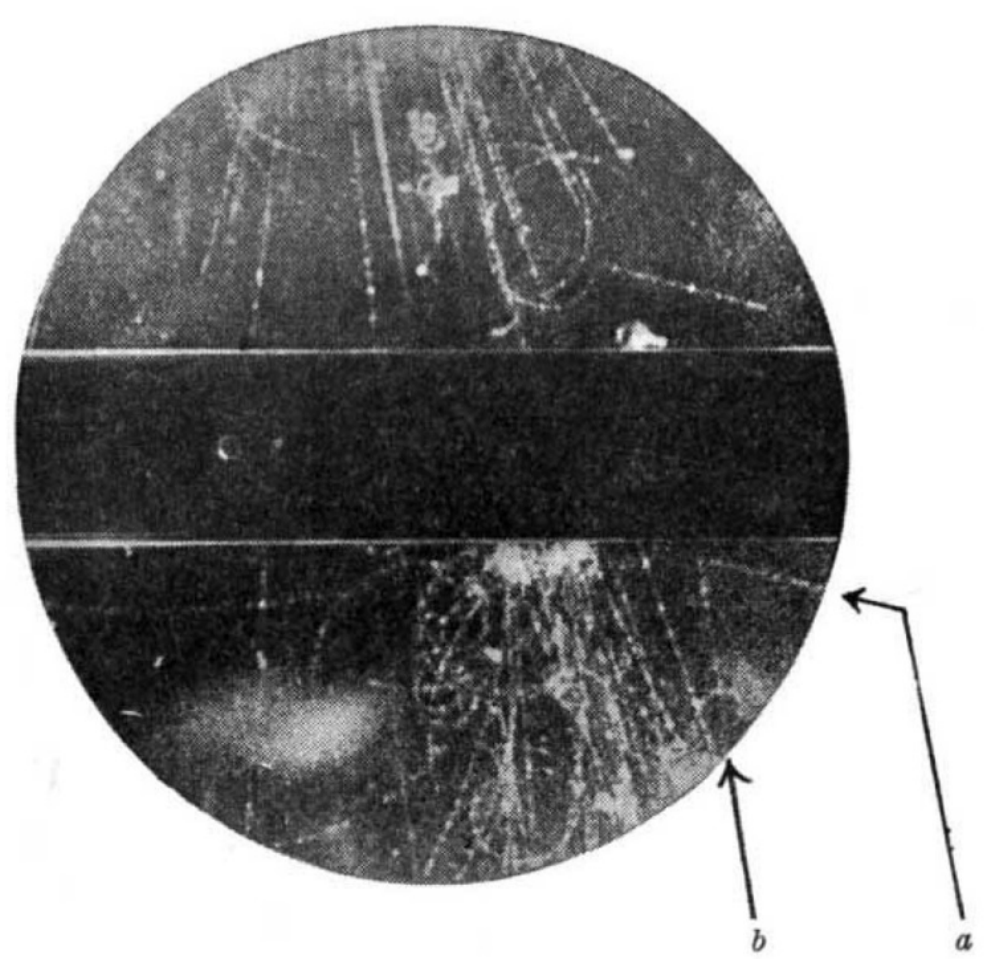

Figure 1.2: The discovery of the first Strange particle, the neutral Kaon, $K^{0}$. In this image, the $K^{0}$ decays into a $\pi^{+}$, and a $\pi^{-}$, respectively labeled 'a' and 'b'. Image from Evidence for the existence of new unstable elementary particles, Nature 160, 855-857 (1947) 


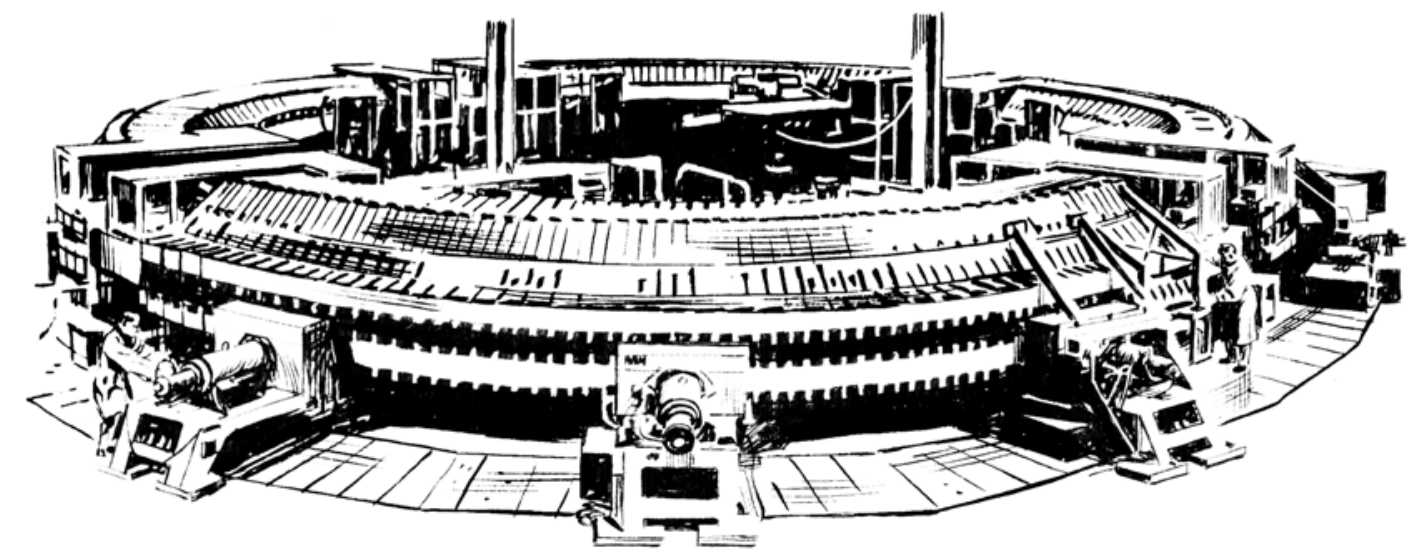

Figure 1.3: The Brookhaven Cosmotron, the first of the modern particle accelerators, and the first to reach the $\mathrm{GeV}$ scale. So-called because its targeted $3 \mathrm{GeV}$ beam energy mimicked that of cosmic rays. Sketch by Pearson Scott Foresman, in the public domain. 
and it was a party. A new property called strangeness was posited to explain the difference in time scales of their production $\left(\sim 10^{-23}\right.$ seconds $)$ and decay $\left(\sim 10^{-10}\right.$ seconds $)^{7}$.

With the discovery of strangeness, Murray Gell-Mann was able to combine charge and strangeness together to create a 'periodic table' of sorts describing the relationships and creating the first catalog of baryons and mesons. He called it the Eightfold Way [8], and introduced it in 1961 [9]. Two example arrangements, the spin $\frac{1}{2}$ baryon octet and the meson octet, can be seen in Figures 1.4 and 1.5. Other shapes include triangles as well as stacked geometric shapes known as supermultiplets.

The Eightfold Way cemented itself as a serious theory when Gell-Mann used it to predict the existence of the Omega Minus $\left(\Omega^{-}\right)^{8}$.

\subsubsection{Quarks}

In technical language, these geometrical shapes can be viewed as representations of the $S U(3)$ group, whose transformations generate the possible multiplets, each with distinct quantum numbers. The Eightfold Way started Gell-Mann on a path that lead him to the Quark Model in order to explain why these geometric shapes can describe the relationships between the baryons and mesons. Gell-Mann noticed that the 'particles' of the $S U(3)$ representations could be described by using certain combinations of the fundamental representation of $S U(3)$. Inspired by

\footnotetext{
${ }^{7}$ It is now understood that a strange particle is created via the strong force, and decays via the weak force.

${ }^{8}$ This was a very big deal.
} 
James Joyce's Finnegan's Wake, he named them quarks. The three flavors of quarks, (up, down, strange), combine to form the mesons and baryons. Mesons can be described as a combination of a quark-antiquark pair, and Baryons can be described as a combination of three quarks.

\subsubsection{Color Charge}

Almost immediately after putting forward the quark model, it was realized that having 3 identical fermions, (as with the 3 strange quarks in the $\Omega^{-}$), violated the Pauli Exclusion Principle, which, as stated earlier, prevented particles with identical quantum numbers from existing in the same state. The (now obvious) solution to this quandary was to add another quantum number that uniquely identified the three strange quarks in the $\Omega^{-}$. This new quantum number was called color, with each quark having its own special charge called color charge $^{9}$. The labels for the charge are red, green, and blue. All particles are colorless, meaning a quark bound state must be 'neutral' in color - i.e. a proton that is composed of three quarks must contain a red quark, a blue quark, and a green quark. A meson like the $\pi^{0}$ must contain, for example, an anti-blue and a blue quark. These concepts were formulated into a theory known as quantum chromodynamics (QCD).

${ }^{9}$ Color charge is a separate charge from classical charge. Quarks also have classical charge, though its magnitude is fractions of a single unit, called fractional charge. 


\subsubsection{GWS and the Vector Bosons}

The next major contribution to particle physics came in the form of the unified gauge theory $S U_{L}(2) \times U(1)$ known as the Glashow-Weinberg-Salam, or Electroweak theory. The authors were awarded the 1979 Nobel Prize in physics for their contributions. It had been postulated prior that there was a mediator particle involved in weak interactions, but it wasn't until the GWS theory that experimentalists at the European Organization for Nuclear Research (CERN) were given enough information to be able to design an experiment to look for these mediators. The GWS theory is one of the most successful theories in physics, predicting and leading to the observations of many important physics results, from neutral currents in 1973, to the discovery of the charm quark at the Stanford Linear Accelerator Center (SLAC) in 1974, the bottom quark at Fermilab in 1977, the aforementioned tau lepton at SLAC in 1975, and the top quark at Fermilab in 1995. Arguably, its most important contribution was the prediction and observation of the intermediate vector bosons at CERN/SPS in 1983: the $W^{+}, W^{-}$, and $Z^{0}$ bosons.

\subsubsection{The Higgs Boson}

In 2012, one of the most important discoveries ever made in particle physics occurred at CERN. First theorized in 1964 to explain why the particles of the Standard Model have mass, the Higgs Boson resonance was teased out of data by the CMS and ATLAS collaborations, winning its theorizers the Nobel Prize in Physics, and creating profound implications for its $125 \mathrm{GeV} / c^{2}$ mass $[10,11]$. Now is a very 
exciting time while we wait for the properties of the Higgs to be uncovered.

\subsubsection{The Present}

It is this history that brings us to now. Our theories are older and wiser. Our experiments are more advanced and more fine-tuned. Our knowledge is deeper than at any point in the past. We are asking some of the most fundamental questions that can be asked about our existence. Some of the most important questions include: Why does the universe prefer matter over anti-matter? What is dark matter and dark energy? How did the universe begin? What is the fate of the universe?

It is only with a strong partnership between theoretical physics and experimental physics that these answers can be found. Current contenders that shed light on these questions include SUSY, (Supersymmetry), a theory which requires the existence of a 'super partner' for every existing particle. Though there has not yet been any evidence for SUSY at the LHC, many remain hopeful for a discovery during the 2015 run, while others aren't holding their breath.

String Theory has not yet been shown to be a natural theory, and the energy regimes our accelerators currently operate at cannot probe the scales necessary to test String Theory. That being said, it holds great promise for solving a number of the key issues currently facing physics and cosmology.

The largest hole in the Standard Model is the inclusion of gravity, or its lack thereof. Many believe that the graviton, a spin 2 particle that mediates the interaction of particles with spacetime, is the answer. It will require another great feat of scientific 
achievement to solve this problem, and many of us are looking forward to that day.

\subsubsection{The Future}

The field of particle physics is advancing on many fronts and in many labs

around the world. Currently, the hottest action is occurring at CERN in Geneva, Switzerland.

The LHC is preparing to start the 2015 run at nearly twice the beam energy of previous runs, and with higher instantaneous luminosity. This will allow not only more data to be recorded over a given period of time, but also more interesting data, as the energy regime explored advances.

There are several hints at so-called "new physics" in data from the LHC. A new peaking structure first observed in the CDF experiment at Fermilab [12] has been observed in the CMS experiment [13]. This could be evidence of a new exotic meson, that cannot be explained with current QCD theory. The LHCb collaboration has observed the existence of an exotic hadron not predicted by the Standard Model [14]. This state is a four-quark state, referred to as a tetraquark, and goes against the current theory that quarks only exist in pairs and triplets. LHCb has also observed an asymmetry in the production of muons, taus, and electrons from b-quarks. According to the Standard Model, a b-quark can decay into two leptons and a hadron, with no preference on the flavor of the leptons produced. In other words, the probability of getting two muons or two electrons or two taus is predicted to be exactly the same. LHCb has seen, in preliminary results, that electrons are produced $25 \%$ more 


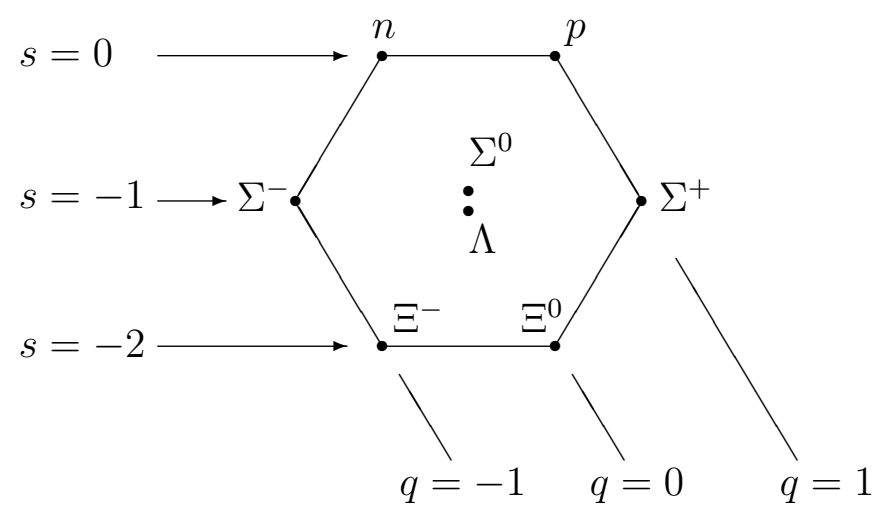

Figure 1.4: The Baryon Octet

of the time, hinting at possible new physics. Like all particle physicists, the LHCb collaboration will have to wait for the 2015 LHC run in order to continue to probe for new physics.

The LHC research program is expected to last at least another 20 years, with several planned upgrades and a goal of delivering $3000 \mathrm{fb}^{-1}$ of data ${ }^{10}$ by 2035 .

Beyond the LHC, physicists are preparing for the next big experiment, the International Linear Collider, or ILC [15]. Briefly, the ILC is being designed to complement the LHC by colliding electrons and positrons ${ }^{11}$.

${ }^{10}$ The unit $\mathrm{fb}^{-1}$ is pronounced inverse femtobarn. More on this in section 1.3.2.

${ }^{11}$ The requirement that it be a linear collider, i.e., a long, straight tube is to limit the energy loss due to Bremsstrahlung or Braking Radiation. This is energy radiated by charged particles when forced on a curved path, or decelerated. Of course, it is a big problem when trying to achieve high energy particle beams, if with every turn energy is being radiated away. At the LHC, protons radiate about $6.7 \mathrm{KeV}$ per turn. The energy loss by a particle accelerated in a circle is $\propto E^{4} R^{-1} m^{-4}$, meaning a heavier particle like the proton loses much less energy than an electron. 


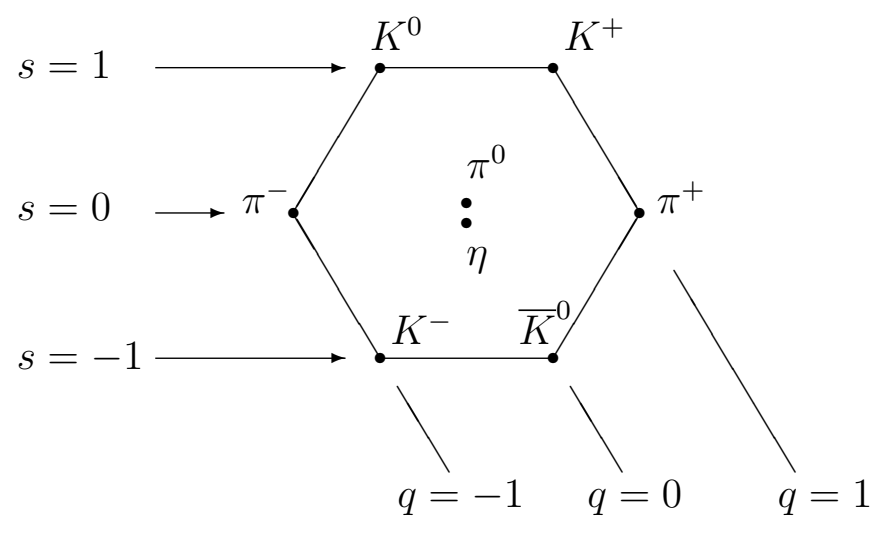

Figure 1.5: The Meson Octet

\subsection{Particle Physics Pertinent to this Analysis}

Though the science of particle physics is far too broad to cover completely in this brief introduction, there are a few necessary concepts that must be understood by the reader in order to appreciate the results described in this thesis.

\subsubsection{Cross Section in Detector-Based Particle Physics}

At the LHC, two oppositely-aligned bunches of protons are collided into each other. When a collision occurs and is recorded by the CMS detector, it is called an event. Physicists use the concept of cross section to describe the probability of a particular event to occur. Every time a collision happens, it is possible for any number of reactions to be produced. If a physicist can know what the probability for a certain process to occur is, they can estimate how many events will need to be recorded in order to make a significant measurement.

The cross section of a particular process depends on the properties of the 
particles being collided and the different interactions they can have, as well as the current of the particle beam, referred to as its instantaneous luminosity, $\mathcal{L}$. The beam luminosity describes the number of particles traveling per unit time per unit area down the beam pipe.

Cross section is measured in units of barns, where 1 barn $=1 \times 10^{-24} \mathrm{~cm}^{2}$. Generally, cross section is quoted in picobarns (pb), or femtobarns ( $\mathrm{fb}$ ).

Often referred to as the fundamental equation of experimental particle physics, the event rate is given by the following equation:

$$
N_{\text {events/second }}=\mathcal{L} \sigma \epsilon
$$

where ' $\mathrm{N}$ ' is the number of events generated per second, ' $\mathcal{L}$ ' is the instantaneous luminosity of the beam, and ' $\epsilon$ ' is the total efficiency for the process.

\subsubsection{An Example}

Take for example the production of a Majorana neutrino with a mass of 200 $\mathrm{GeV} / c^{2}$. The cross section for this process is $0.13 \mathrm{pb}$. The design luminosity of the LHC proton-proton beam is $1 \times 10^{34} \mathrm{~cm}^{-2} \mathrm{~s}^{-1}$.

Assuming optimal LHC design characteristics, and an efficiency of 1 , the event rate for this process is:

$$
\begin{gathered}
N_{\text {events } / \text { second }}=\left(1 \times 10^{34} \mathrm{~cm}^{-2} \mathrm{~s}^{-1}\right) \cdot\left(0.13 \times 10^{-12} \text { barns } \cdot 10^{-24} \mathrm{~cm}^{2} / \text { barn }\right) \\
N_{\text {events } / \text { second }}=0.0013 \rightarrow 769 \text { seconds } / \text { event } .
\end{gathered}
$$


The total number of events can be determined by integrating both sides:

$$
N_{\text {events }}=\sigma \times \mathcal{L}_{\text {int }}
$$

where $\mathcal{L}_{\text {int }}$ is the integrated luminosity, or the total amount of beam delivered. This is regularly used to refer to the amount of data collected by collider experiments, and is generally quoted in units of inverse femtobarns, or $\mathrm{fb}^{-1}$.

\subsubsection{Energy Resolution of a Calorimeter}

The energy resolution of a calorimeter can be described as a quantification of how finely the detector can distinguish between similar energies. Ideally, every pion with $100 \mathrm{GeV}$ of energy would show up in the detector as a blip at $100 \mathrm{GeV}$. In reality, however, the peak has some width and generally has the shape of a Gaussian distribution, as seen in Figure 1.6.

The energy resolution of a detector is defined as the ratio of the width of the measured energy distribution to its mean, written as:

$$
\text { Energy Resolution }=\frac{\sigma(E)}{E}
$$

Equation 1.5 is normally expressed as a percentage.

As an example, see Figure 1.7 which shows a simple Gaussian fit to the response of $150 \mathrm{GeV} / c$ pions in HF. Also displayed is the sigma and mean of the fit, allowing one to calculate the energy resolution of $150 \mathrm{GeV} / c$ pions in $\mathrm{HF}$ :

$$
\text { Energy Resolution }=\frac{\sigma(E)}{E}=\frac{24.13}{132.1}=18.3 \%
$$




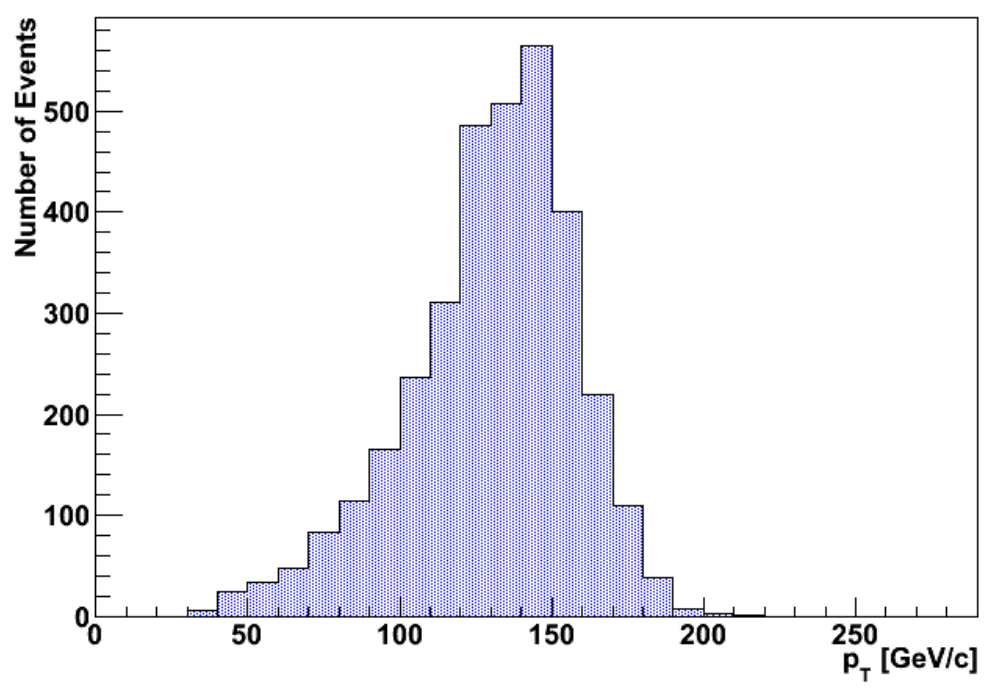

Figure 1.6: HF detector response to $150 \mathrm{GeV} / c$ pions.

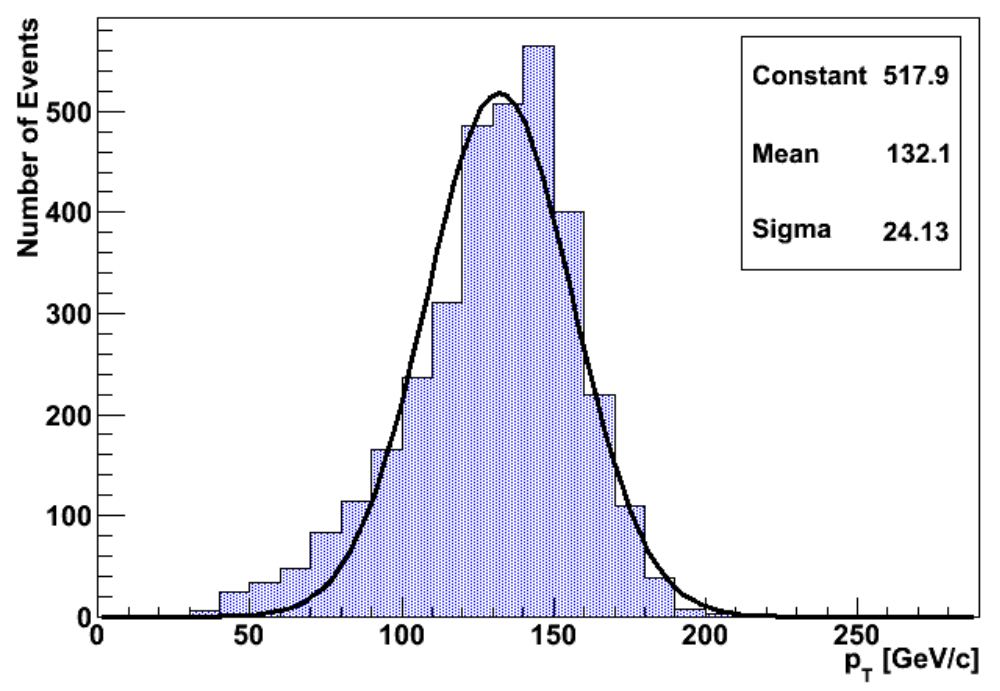

Figure 1.7: A Gaussian fit of the HF detector response to $150 \mathrm{GeV} / c$ pions. 


\section{CHAPTER 2 \\ APPARATUS}

This chapter describes the experimental apparatus and tools used to perform the analyses in this thesis. For a fantastically detailed treatment of the LHC machine, see [16]. See [17] for a thorough description of the physics and design of accelerators.

\section{$2.1 \quad$ Introduction}

The experimental apparatus described herein are located at CERN, on the outskirts of Geneva, Switzerland and across the borders of France and Switzerland. CERN was first established in 1954, recently celebrating its 60th anniversary. CERN has a diverse research program, all in support of studying the fundamental aspects of the universe, with an emphasis on providing state-of-the-art accelerator technology to its facilities and the world. CERN has many secondary sites outside its main campus, the largest of which is the Prevessin site where the University of Iowa CMS group conducts its fixed target research activities. See Figure 2.1 for a map of the CERN site.

CERN is responsible for many technological achievements, including most famously the development of the World-Wide-Web by Tim Berners-Lee[18]. Many of the most important discoveries in particle physics have occurred at CERN, including the discovery of neutral currents[19], the $\mathrm{W}$ and $\mathrm{Z}$ bosons[20][21], the first creation of anti-hydrogen, the discovery of $\mathrm{CP}$ violation, and of course the observation of the Higgs Boson. 


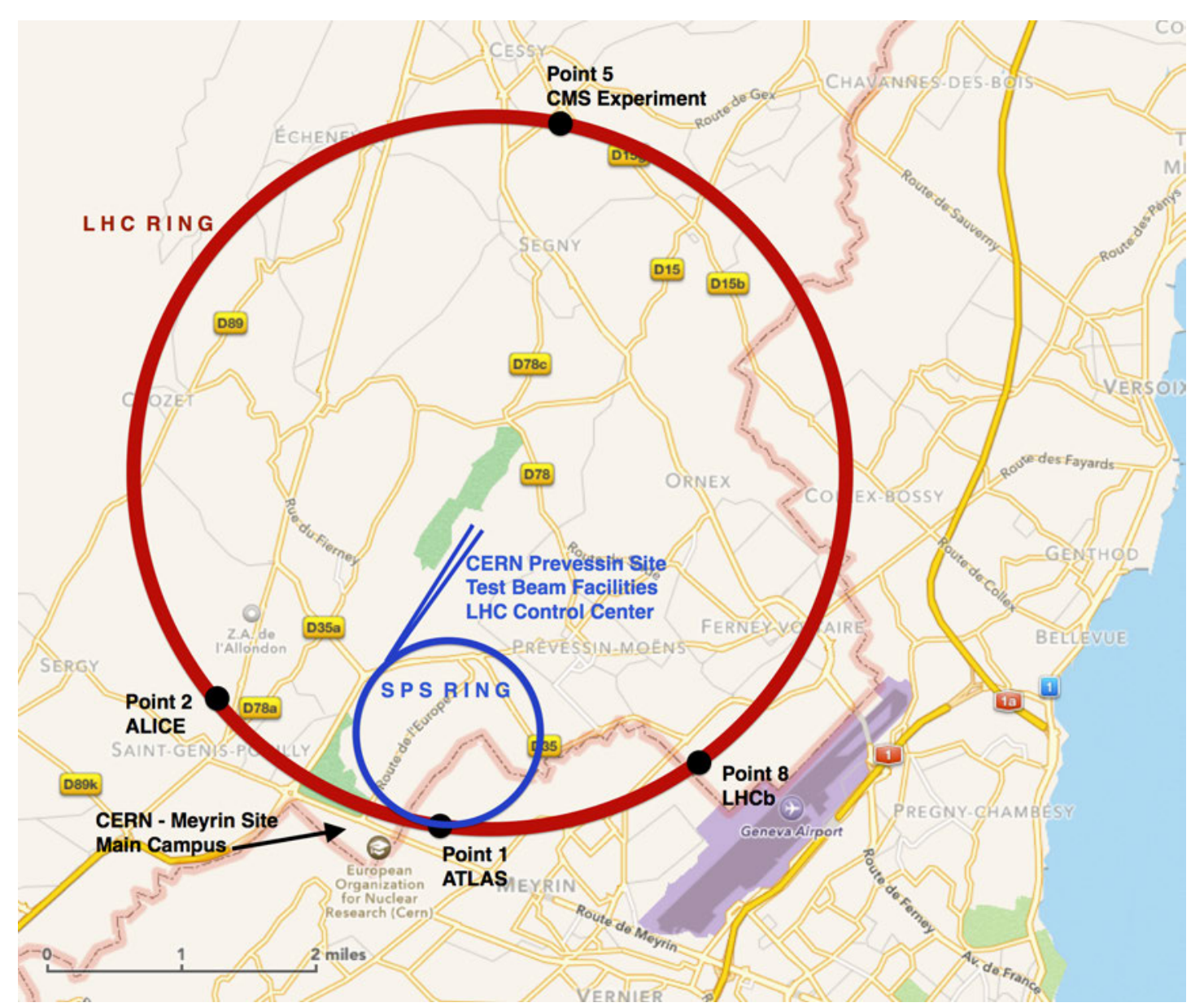

Figure 2.1: Map of the CERN site. Map image courtesy of Apple Maps, Apple, Inc.

The areas of most concern for this thesis include the LHC accelerator complex and the CMS experiment.

\subsection{The Large Hadron Collider}

In order to study the extremely short lived particles allowed by the universe, physicists need to recreate the exotic and controlled environments necessary to produce them. The Large Hadron Collider at CERN was designed to collide protons at a center-of-mass energy of 14 tera-electronvolts $(\mathrm{TeV})$. Four separate collision halls 
were built to house four separate experiments: two general purpose detectors, ATLAS and CMS, and 2 specialty detectors, the b-quark-studying LHCb and the Pb-Pb collision-studying ALICE. Dedicated heavy ion runs are allocated during scheduled beam time, during which the protons in the beam pipe are evacuated and replaced with lead ions in order to study quark-gluon plasmas.

\subsubsection{Principles of Design}

The LHC's central feature is its accelerator system composed of a closed ring of 1232 superconducting dipole magnets supercooled to 1.9 Kelvin, evacuated to $10^{-10}$ Torr, and positioned around the ring to sub-centimeter precision, totaling $27 \mathrm{~km}$. Figure 2.2 shows a cross section of the main accelerator component, the LHC dipole magnet. Figure 2.3 shows the incredible precision required of the B-field in order to steer the protons. Table 2.1 lists the design characteristics of the LHC.

Figure 2.5 schematizes the CERN accelerator complex, including the dates that each accelerator came online. The research accelerators of the past become the booster accelerators of the future.

\subsubsection{Proton Injection}

Before any collisions can happen, a complex process of getting the protons to the interaction point must occur. This sophisticated process begins with a tank of hydrogen gas, and not just any hydrogen gas, but hydrogen gas so pure the impurities are measured in parts per billion. A $100 \mathrm{kV}$ duoplasmatron collects the hydrogen gas and feeds it to a cathode chamber to strip off the electrons, yielding $\approx 70 \%$ protons. 


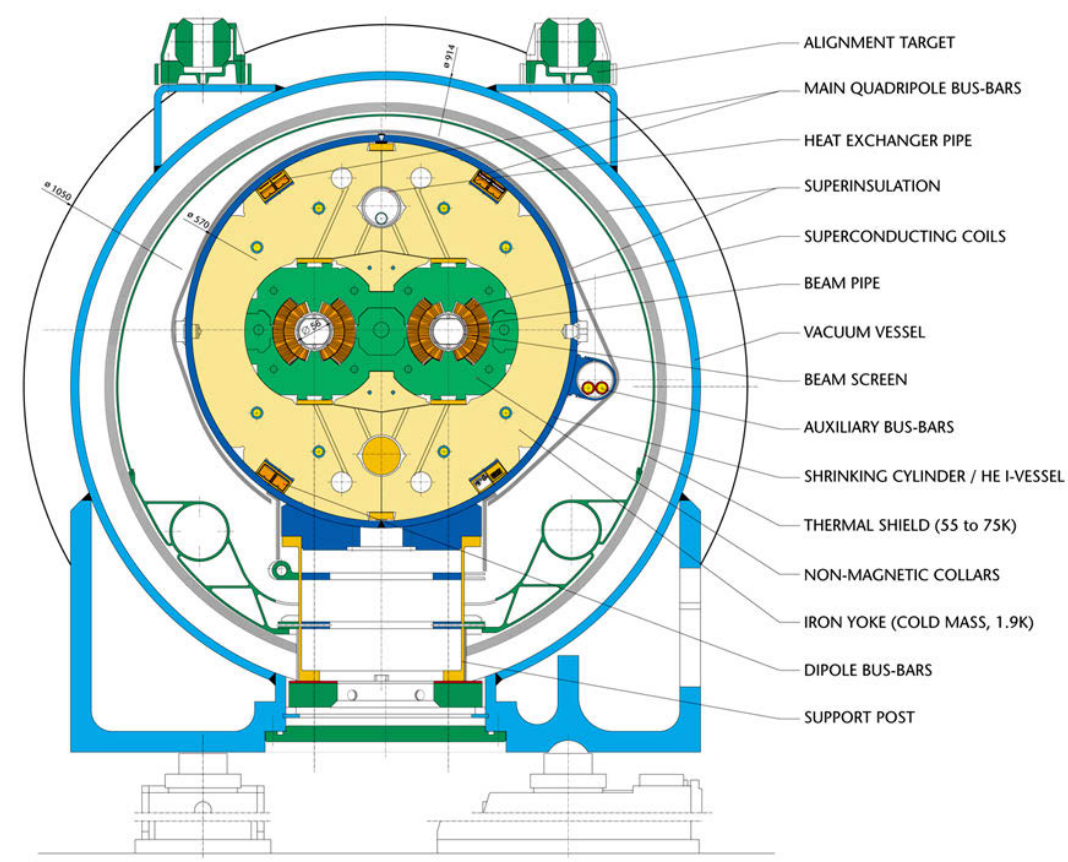

Figure 2.2: LHC Dipole Magnet Cross Section. [CERN]
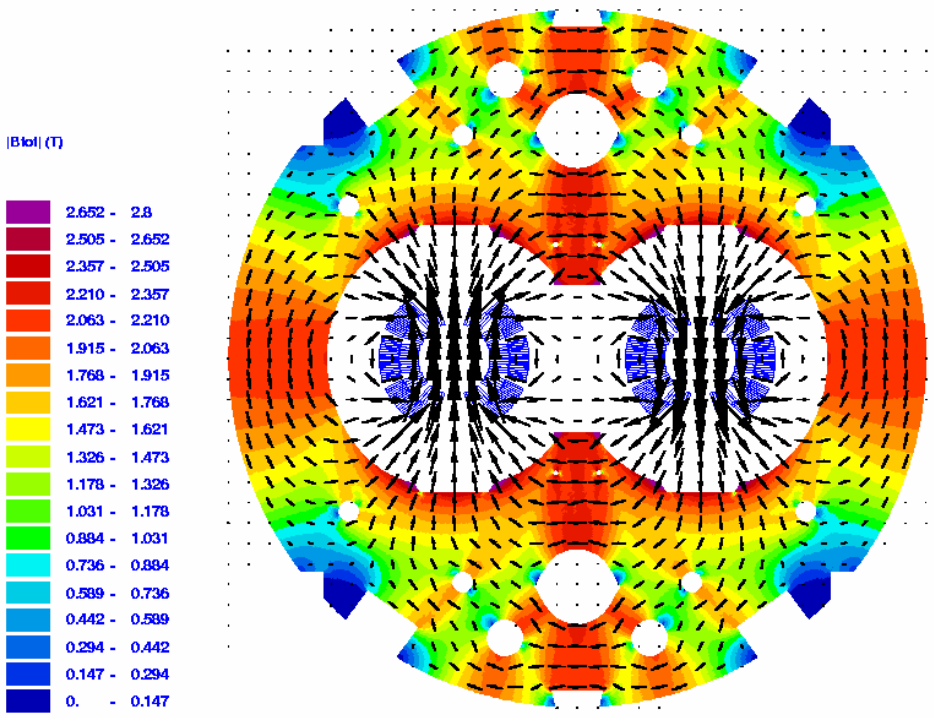

Figure 2.3: LHC Dipole Magnet B-Field. [CERN] 


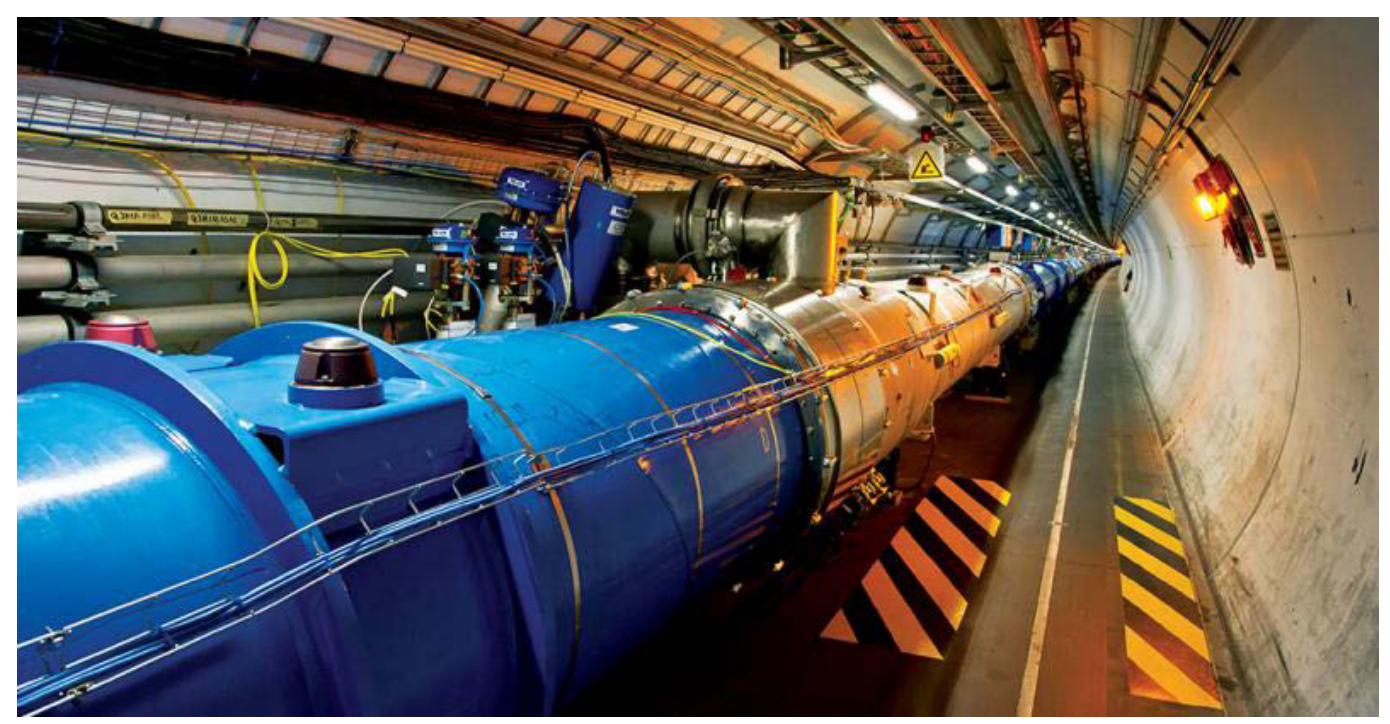

Figure 2.4: Inside the LHC Accelerator Tunnel. [CERN]

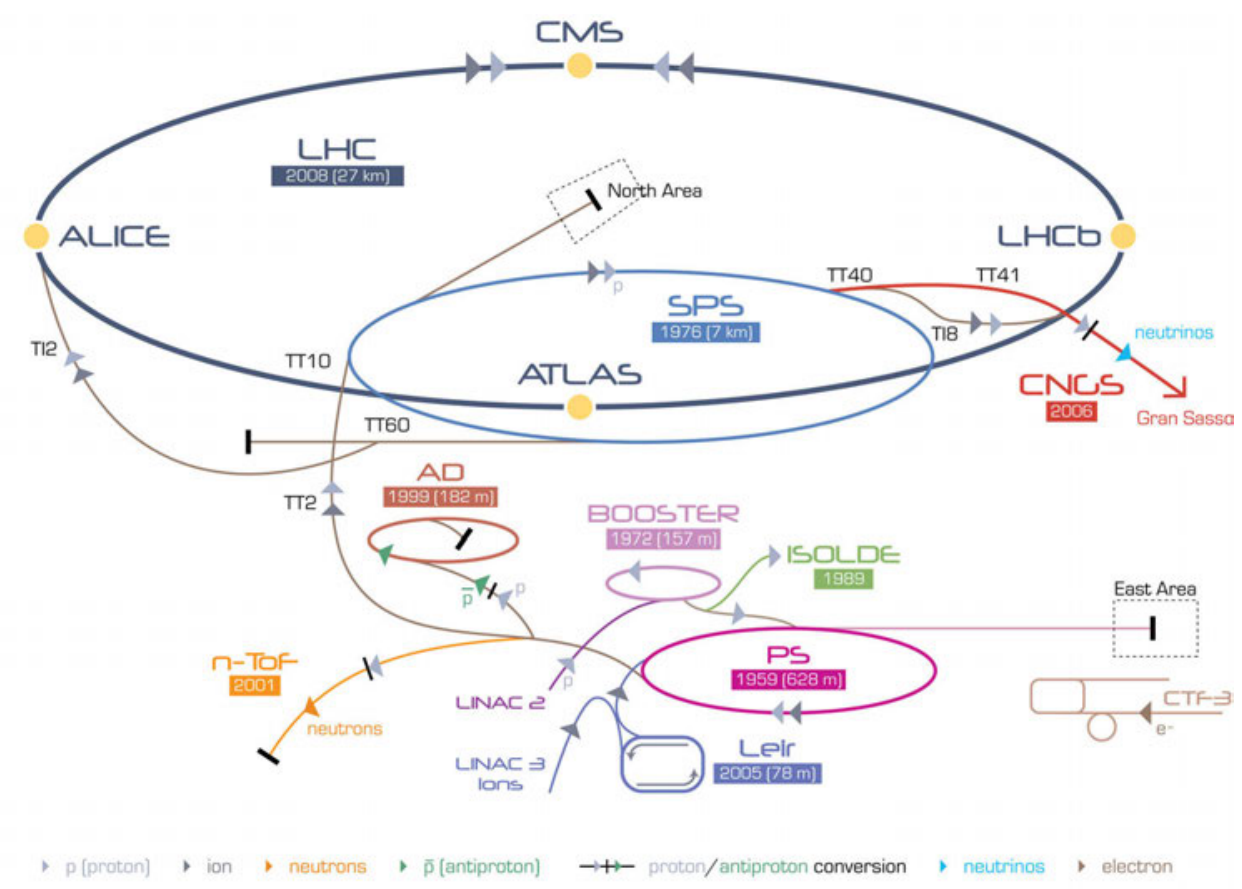

LHC Large Hadron Collider SPS Super Proton Synchrotron PS Proton Synchrotron

AD Antiproton Decelerator CTF-3 Clic Test Facility CNCS Cern Neutrinos to Gran Sasso ISOLDE Isotope Separator OnLine DEvice LEIR Low Energy lon Ring LINAC LINear ACcelerator n-ToF Neutrons Time Of Flight

Figure 2.5: The CERN Accelerator Complex. [CERN] 
Table 2.1: Summary of the LHC design characteristics.

\begin{tabular}{|c|c|}
\hline \multicolumn{2}{|c|}{ Machine Properties } \\
\hline Number of Accelerator Stages & \\
\hline Circumference & $27 \mathrm{~km}$ \\
\hline Operating Temperature & $1.9^{\circ} \mathrm{K}$ \\
\hline Energy Usage & $120 \mathrm{MW}$ \\
\hline Liquid Nitrogen Contained & 10,800 Tons \\
\hline Liquid Helium Contained & 60 Tons \\
\hline Tunnel Gradient & $1.4 \%$ \\
\hline Beam Interlock Safety Signals & Over 10,000 \\
\hline \multicolumn{2}{|c|}{ Beam Properties } \\
\hline Collision Energy & $7+7 \mathrm{TeV}$ \\
\hline$\gamma$ Factor & 7461 \\
\hline Luminosity & $10^{34} \mathrm{~cm}^{-2} \mathrm{~s}^{-1}$ \\
\hline Injection Energy & $450 \mathrm{GeV}$ \\
\hline Beam Crossing Points & 4 \\
\hline Number of Bunches & 2800 \\
\hline Number of Protons Per Bunch & $1.5 \times 10^{11}$ \\
\hline Time Spacing Per Bunch & $25 \mathrm{~ns}$ \\
\hline Beam Crossing Rate & $40 \mathrm{MHz}$ \\
\hline Beam Current & $2 \times 0.58 \mathrm{~A}$ \\
\hline Stored Energy & $2 \times 334 \mathrm{MJ}$ \\
\hline Bunch Width at Intersection & $16.7 \mu m^{2}$ \\
\hline Bunch Length at Intersection & $11.24 \mathrm{~cm}$ \\
\hline Amount of Steel for Magnet Yokes & 55,000 Tons \\
\hline Beam Velocity Magnitude & $0.999999991 \mathrm{c} c$ \\
\hline \multicolumn{2}{|c|}{ Magnet Properties } \\
\hline Dipole Magnetic Field Strength & $8.4 \mathrm{~T}$ \\
\hline Number of Main Dipole Magnets & 1232 \\
\hline Number of Quadrupole Magnets & 858 \\
\hline Number of Correcting Magnets & 6208 \\
\hline Total Number of Magnets & 9300 \\
\hline Energy Stored Per Magnet & $7 \mathrm{MJ}$ \\
\hline
\end{tabular}


Magnetic fields compress and inject the protons at roughly $4,000 \mathrm{~km} / \mathrm{s}(0.01 c)$ into the LINAC2; see Figure 2.5.

The protons then leave the LINAC2 with an energy of $50 \mathrm{MeV}(0.31 c)$ and enter the Proton Synchrotron Booster (PSB). The PSB accelerates the protons to $1.4 \mathrm{GeV}(0.92 c)^{1}$ and injects the protons into the Proton Synchrotron (PS $)^{2}$. The PS accelerates the protons to $25 \mathrm{GeV}(0.9993 c)^{3}$.

The PS then hands the protons off to the Super Proton Synchrotron (SPS) ${ }^{4}$ which accelerates the protons to $450 \mathrm{GeV}(0.999998 c)^{5}$.

After the SPS, the protons are injected into the LHC, which during the 2011 run, brought the protons to $3.5 \mathrm{TeV}, 4 \mathrm{TeV}$ during the $2012 \mathrm{run}$, and will bring them to the full design energy of $7 \mathrm{TeV}$ (0.999999991c) during the 2015 run.

\subsubsection{Beam Control}

Controlling the LHC beam is a precise and involved task, with literally thousands of inputs that need to be tuned. I invite the reader to consult $L H C$ Beam Stability and Feedback Control [22] for the methods and techniques beam scientists use to provide clean proton beams to the experiments.

\footnotetext{
${ }^{1}$ At this energy, a proton is about 2.5 times more massive than at rest.

${ }^{2}$ The PS was CERN's first accelerator, brought online in 1959.

${ }^{3} \mathrm{~A}$ proton is now almost 27 times more massive than it is at rest.

${ }^{4}$ First turned on in 1976, this machine was responsible for the discovery of the $\mathrm{W}$ and $\mathrm{Z}$ bosons.

${ }^{5} \mathrm{~A}$ proton is now almost 500 times more massive than it is at rest. Witness to the speed limit of the universe.
} 


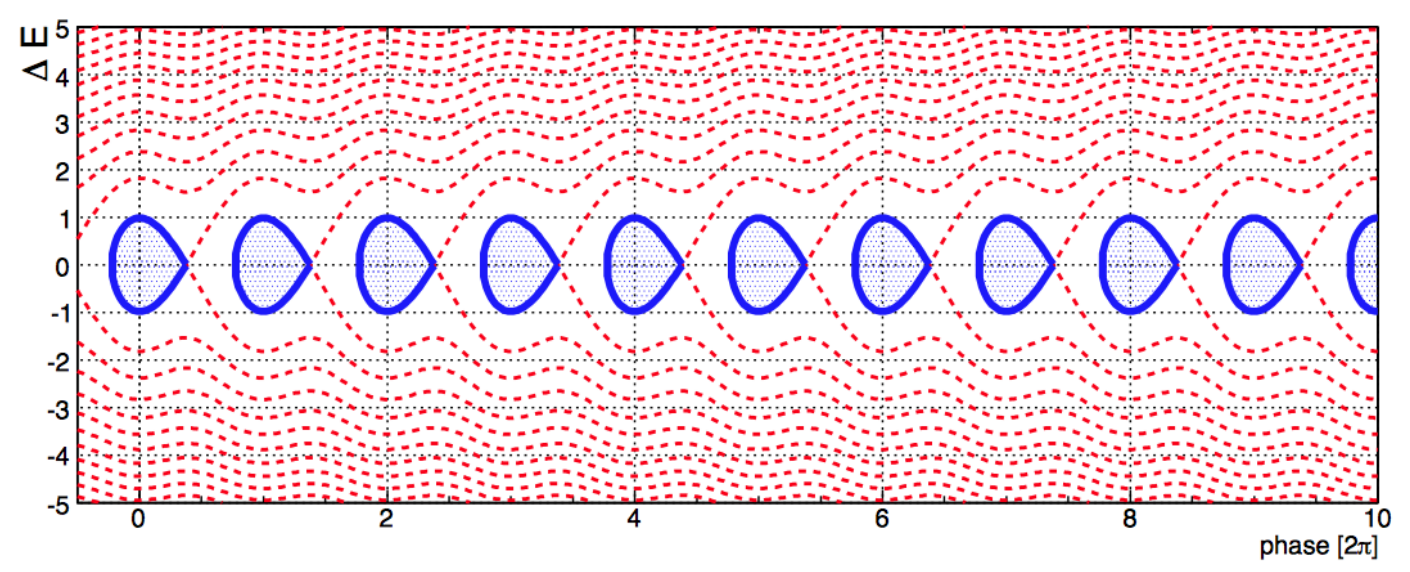

Figure 2.6: Longitudinal phase diagram for an accelerating RF system. [CERN]

The LHC accelerator machine uses a system of alternating dipole and quadrupole magnets to accelerate and focus the proton beam.

Figure 2.6 shows a longitudinal phase diagram for an accelerating RF system. Particles are accelerated in bunches located in the blue region of Figure 2.6, representing stability in phase space.

Some of the more bizarre concerns LHC beam physicists have to consider are thermal heating of the beam-pipe support girders, and distortion of the earth's crust due to lunar and solar gravitational forces. The lunar perturbation affect has a periodicity of $\approx 25$ hours. The solar perturbation period is 24 hours with an effect $\approx 45 \%$ that of the moon. The secondary terrestrial tides result in a local shifting of the ground by $\Delta a / a$ on the order of $10^{-8}$ as shown in Figure 2.7. The largest impact on the LHC occurs during spring tides, when the sun and moon are on the same side of the Earth. This results in a shift of the proton energy which causes the protons 

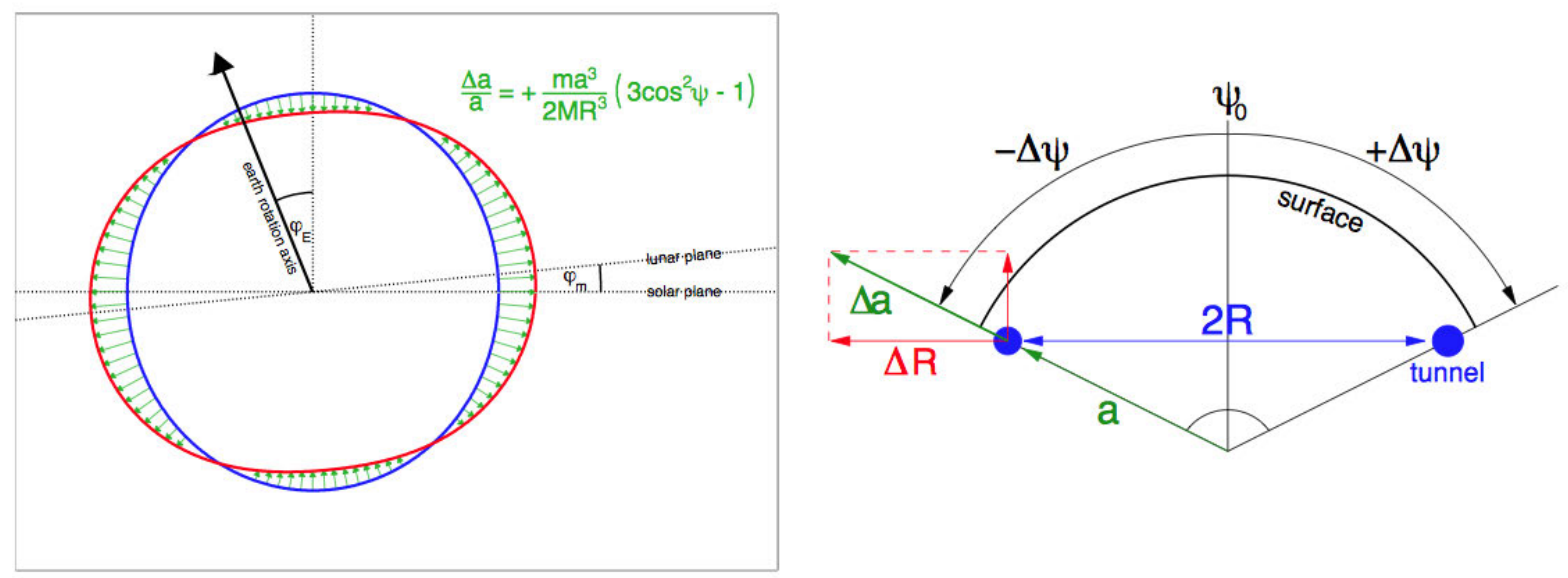

Figure 2.7: Tidal deformation of the Earth's surface (left) and resulting LHC tunnel deformation (right). This diagram is not to scale. [CERN]

to move on a dispersion orbit on the order of $150 \mu \mathrm{m}$. This and other perturbations are compensated with beam stabilization techniques that include machine operator control tools like RF tuning, as well as automatic orbit feedback systems using beam position monitors and dipole corrector magnets. 


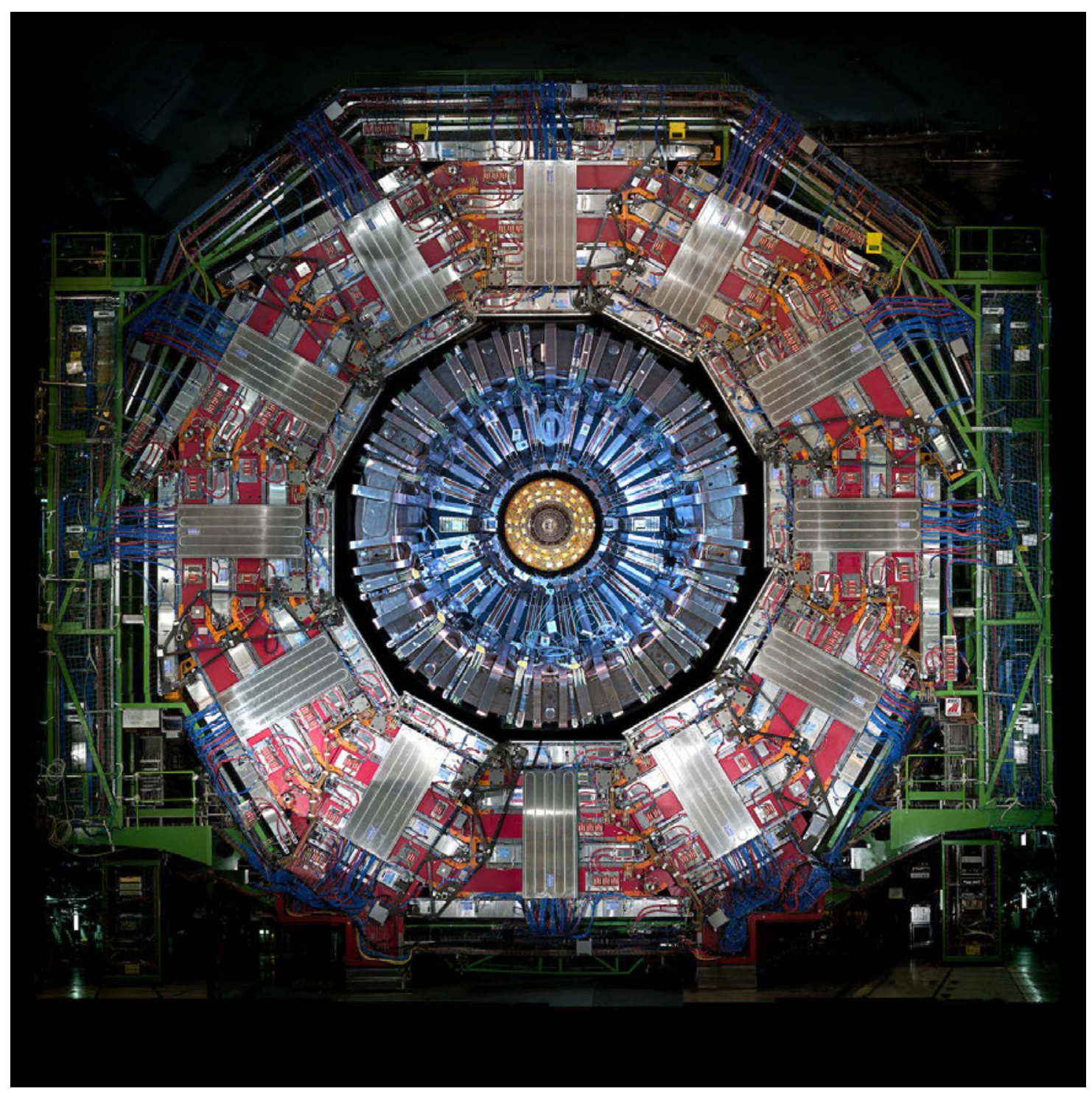

Figure 2.8: The CMS detector in the experimental cavern. [CERN]

\subsection{The Compact Muon Solenoid Detector}

The CMS Detector is the main experimental apparatus responsible for collecting the data used for the analysis chapter of this thesis, and whose detector elements are the subject of the hardware chapter. The Technical Design Report (TDR)[23] of 
the CMS detector is an excellent resource for those interested in a more thorough treatment of the detector elements.

\subsubsection{Principles of Design}

The CMS detector is one of two general purpose detectors, along with ATLAS, on the LHC. CMS is a $4 \pi$ detector, meaning in principle it is able to reconstruct collisions with full $\mathrm{r} / \mathrm{rho} /$ phi coverage around the interaction point. CMS is an initialism for Compact Muon Solenoid:

Compact for its density of detector elements.

Muon due to the large role, both in the physical design and the physics goals, that muons and the muon detectors play.

Solenoid for the massive 4 Tesla magnet system at the heart of the detector.

Figure 2.8 is an image of the CMS detector, without its end caps in place ${ }^{6}$. It is the view of the detector a proton would see, looking right down the beam line. Figure 2.9 is an isometric cutaway view of CMS, with each of its subdetectors labelled.

A common coordinate system is defined in CMS for locating elements in the detector for use within software and for engineering purposes.

- The $z$-axis is defined as the axis along the beam-line, with $z=0$ at the interaction point.

\footnotetext{
${ }^{6} \mathrm{~A}$ main design goal of the CMS collaboration was to ensure, despite its compactedness, easy access to detector elements for upgrade and maintenance. Each slice of CMS can be moved independently by forcing pressurized air through its feet, giving it just enough lift to be pushed around.
} 
- The $x$-axis points towards the center of the LHC.

- The y-axis is defined as straight up from the interaction point.

- The angle $\phi$, referred to as the azimuthal angle, is measured from the $x$-axis in the $x-y$ plane, where $\phi=0$ points towards the center of the LHC ring with radial component $r$.

- The polar angle $\theta$ is defined in the $r z$ plane.

- The pseudorapidity is defined as $\eta=-\ln (\tan (\theta / 2))$, and is useful due to the Lorentz invariance of the opening angle between particles.

The sub-detectors of CMS are the tracker, electromagnetic calorimeter (ECAL), hadronic calorimeter (HCAL), and muon system. As a particle travels from the interaction point through the detector, each detector subsystem is responsible for a part of its reconstruction. See Figure 2.10 for a diagram of the particle flow in CMS and how different particles respond to the different subdetectors.

The tracker system is designed to record precision tracks of the particles to help identify individual particle momentum by precisely fitting their trajectories. The ECAL and HCAL systems are designed to measure the energies of the particles. The muon system has the very difficult job of reconstructing muons. The detector software has to stitch the signals from each of these detectors together to create the reconstructed particle objects used for analysis. 


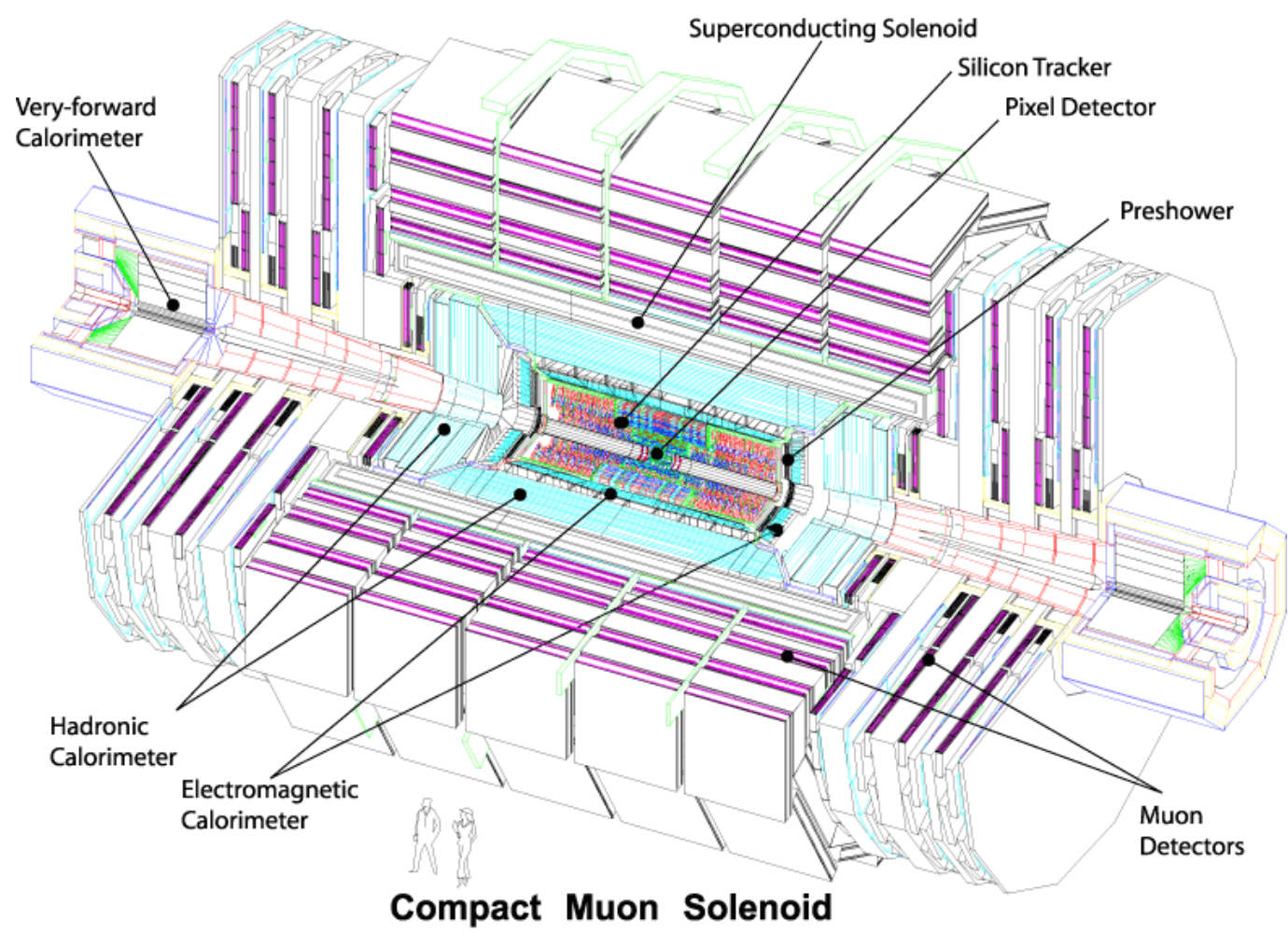

Figure 2.9: Cutaway isometric view of the CMS detector. [CERN]

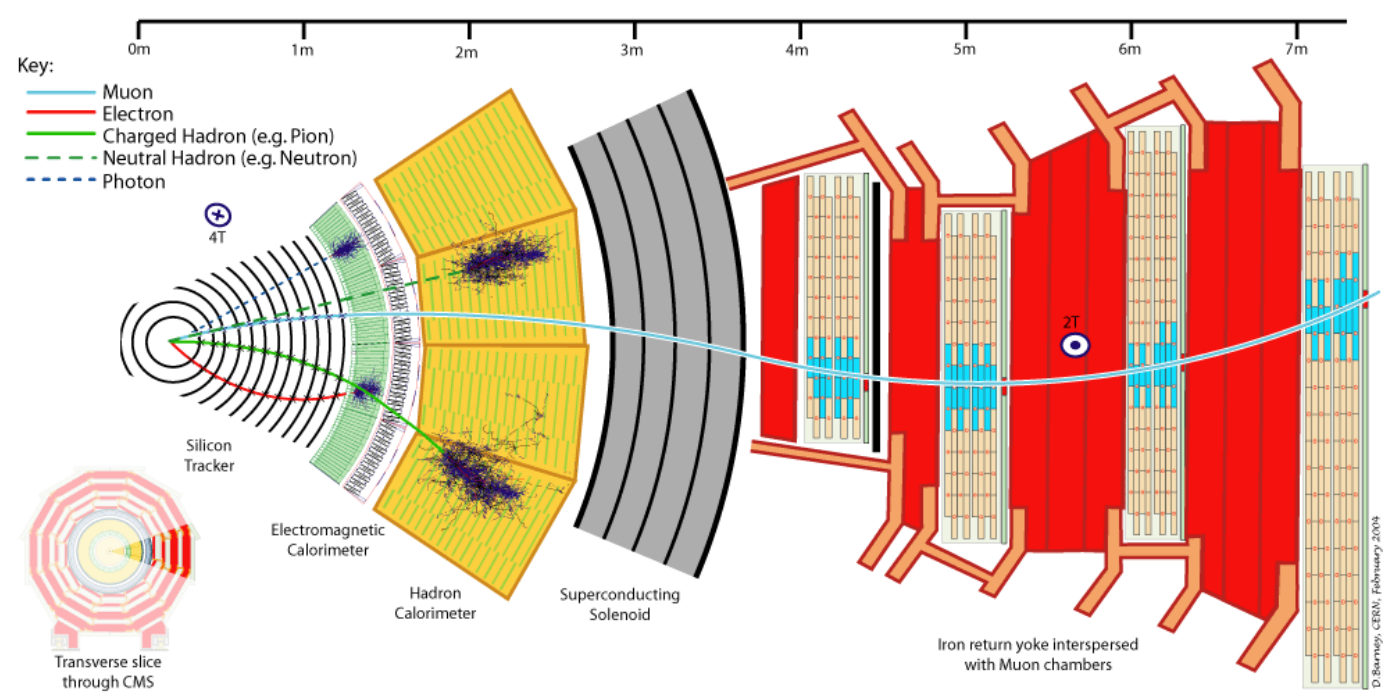

Figure 2.10: Particle flow in the CMS Detector. [CERN] 


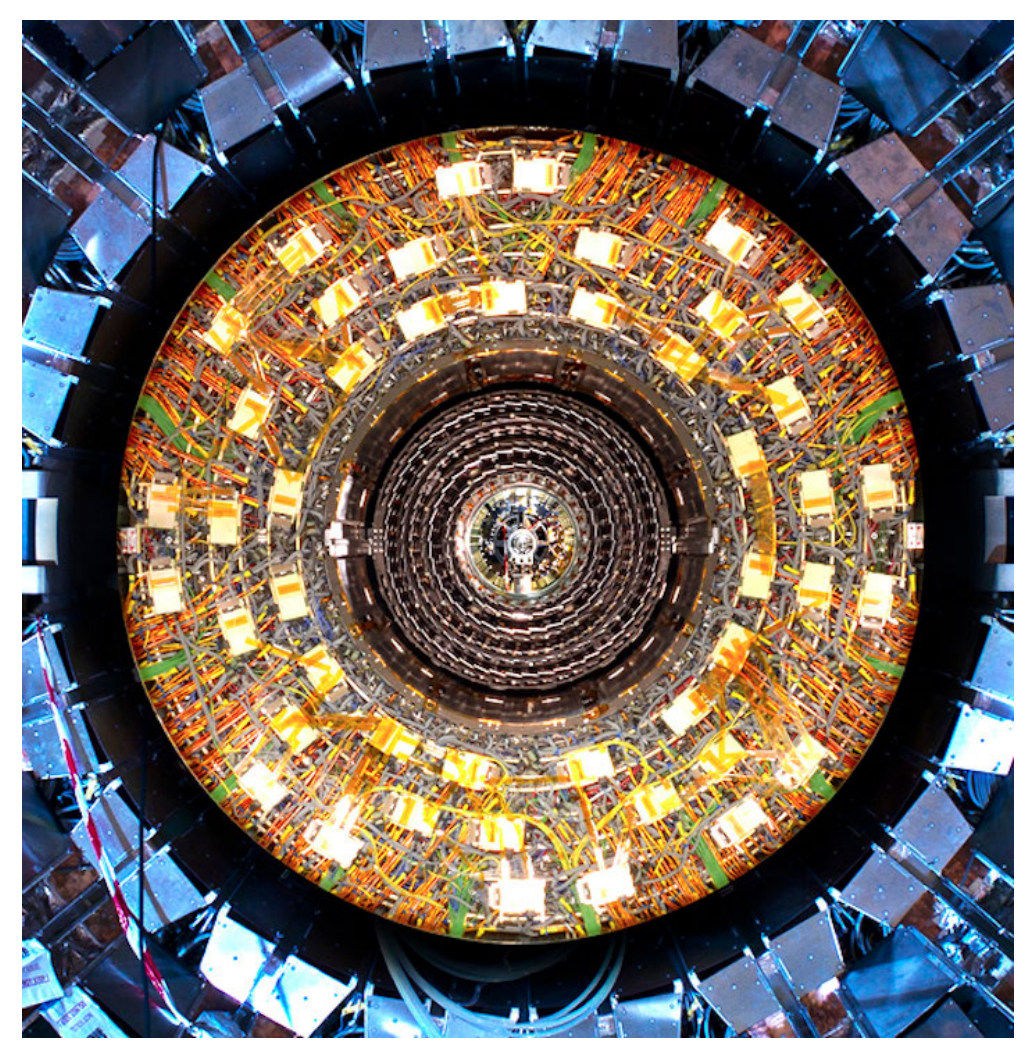

Figure 2.11: The CMS Tracking System viewed along the beam line. [CERN]

\subsubsection{Inner Tracker}

The main goal of the tracking system in CMS is to record the initial trajectories of the particles prior to reaching the calorimeter systems allowing the precise measurement of the particles' paths and momentums. The inner tracker system has a total active area of over $200 \mathrm{~m}^{2}$, and is composed of two main subsystems: the pixel detector (within $0.2 \mathrm{~m}$ of the beam pipe) and the silicon strip detector (between 0.2$1.2 \mathrm{~m}$ from the beam pipe), as seen in Figure 2.11. The pixel detector is composed of 1440 pixel modules totaling 66 million individual pixels. The silicon strip detector 
contains 15,148 modules containing 9.3 million readout channels.

Precise reconstruction of collision vertices is a major tool to limit pileup contamination. Pileup is a term used to describe the amount of extemporaneous particles in a detector beyond those of interest in the main event. Pileup is due to the nature of proton-proton collisions and the high luminosity of the collision environment, causing several interactions in each event. The higher the beam luminosity, the greater the pileup. The tracking system is used to precisely reconstruct interaction vertices, limiting the effects of pileup.

Because of its close proximity to the beam line and the high flux of particles to which it is exposed, the tracker system will be the first CMS sub-detector to be replaced due to radiation damage. Despite the peril of its proximity, its closeness to the interaction point is the tracker system's greatest asset. 


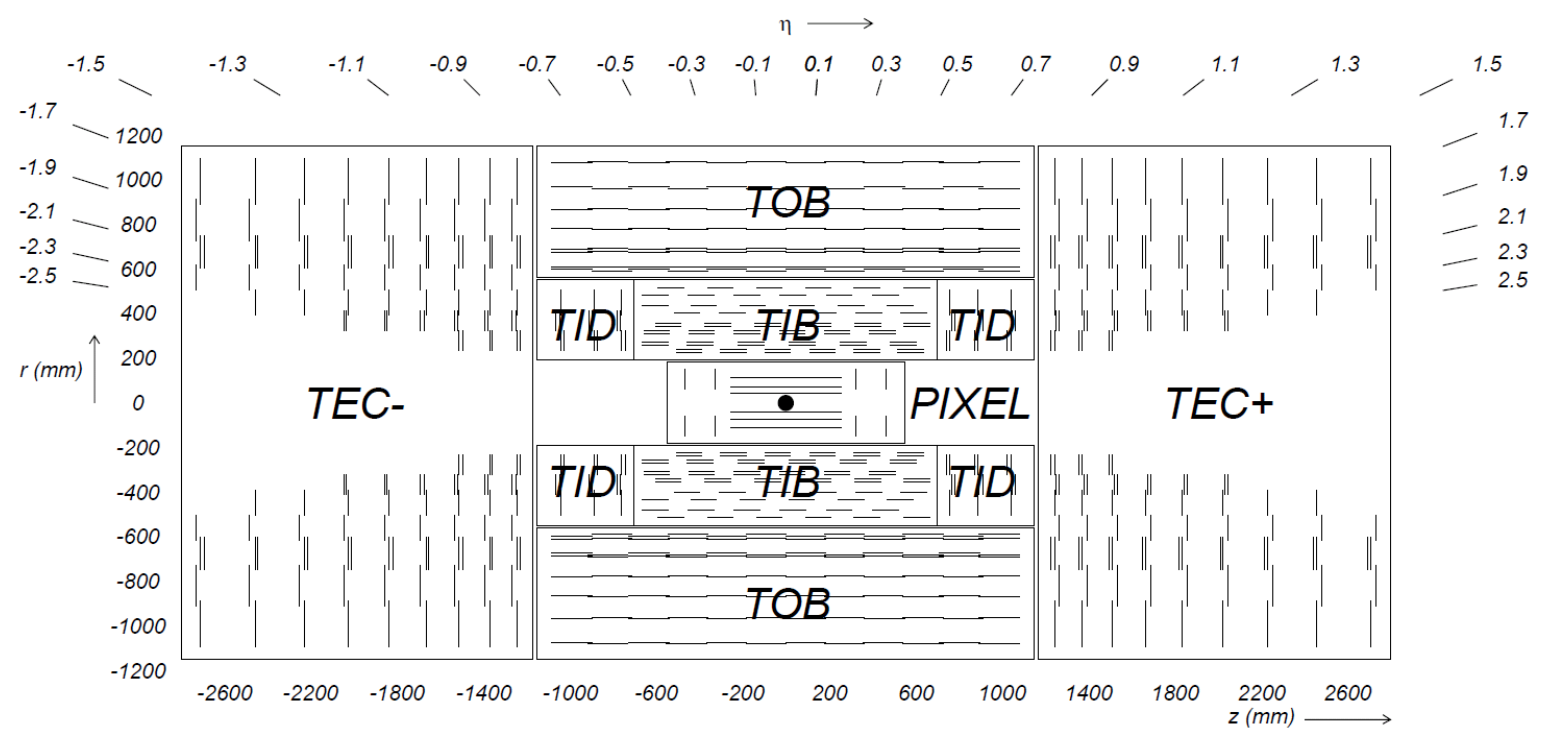

Figure 2.12: The CMS Tracking system shematic. Each line represents a layer of active detector elements. The submodules listed represent the inner barrel (TIB), outer barrel (TOB), inner disc (TID), and endcap regions (TEC). [CERN]

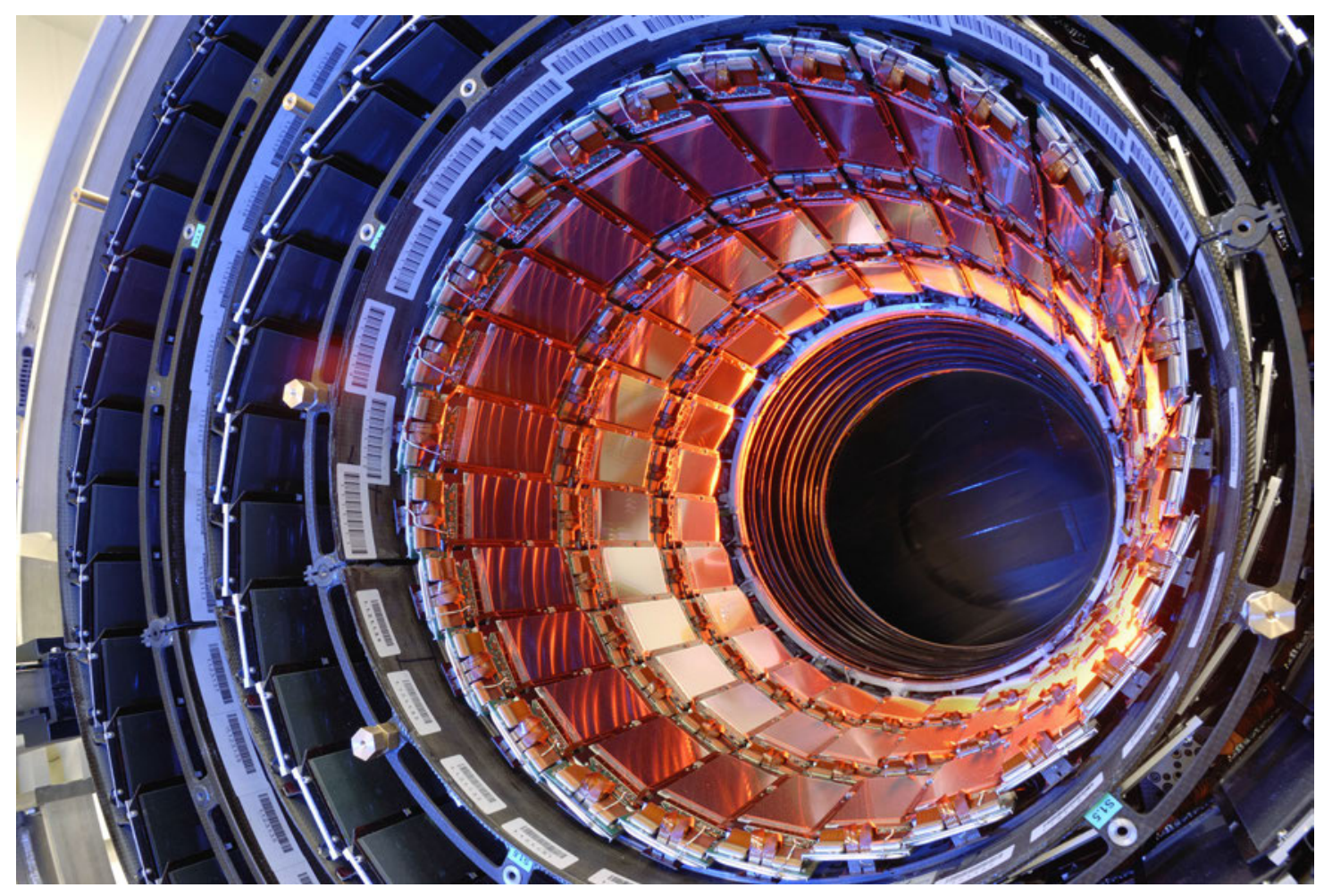

Figure 2.13: The silicon strip inner barrel of the CMS tracking system. [CERN] 


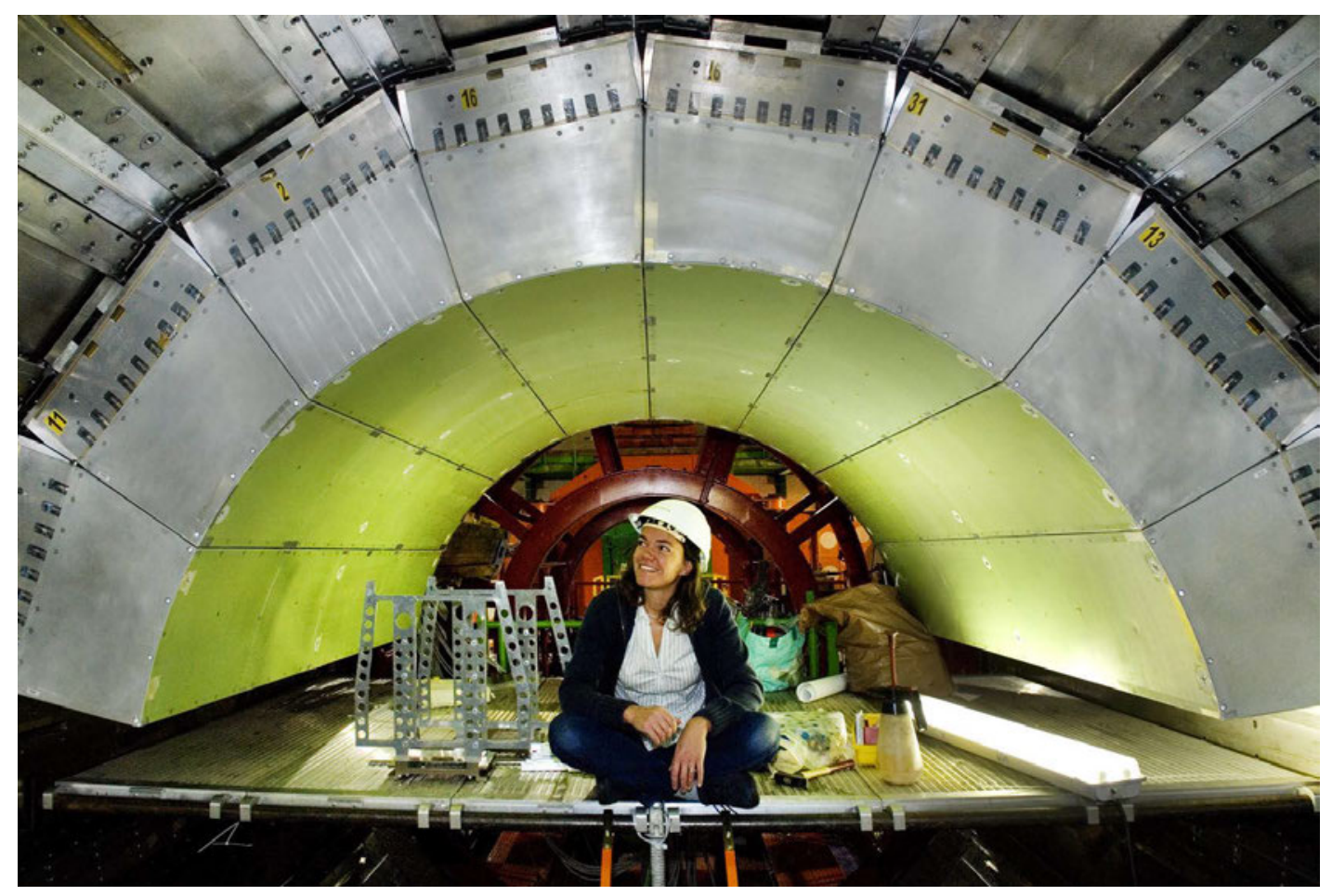

Figure 2.14: Italian physicist Francesca Cavallari beneath the ECAL Barrel. [CERN]

\subsubsection{Electromagnetic Calorimeter}

The ECAL Barrel (EB) system's central detector element is a $2.2 \times 2.2 \times 23$ $\mathrm{cm}^{3}$ lead tungstate $\left(\mathrm{PbWO}_{4}\right)$ crystal, read out by avalanche photo diodes (APDs) with an active area of $5 \times 5 \mathrm{~mm}^{2}$. The response of an APD to an electron changes by $3.8 \% /{ }^{\circ} \mathrm{C}$, requiring a water cooling system in ECAL to maintain a nominal temperature of $18^{\circ} \mathrm{C}$, and be monitored to a precision of $\pm 0.05^{\circ} \mathrm{C}$.

The ECAL Endcap (EE) system also uses lead tungstate crystals with a slightly different geometry, $2.86 \times 2.86 \times 22 \mathrm{~cm}^{3}$, read out with vacuum phototriodes (VPT). 
Figure 2.15 is a schematic view of the ECAL system, showing the 'D' shaped endcaps (each half referred to as a 'Dee'), and the central barrel. The cutaway also shows off the $\mathrm{PbWO}_{4}$ crystal arrangement visible as the finely segmented regions in the upper cutaway of the barrel, and horizontally oriented along the edge of the dee end-cap, on the left of the schematic. Notice the change in gradient of the crystals as they get further from the interaction point (the center of the 3D object).

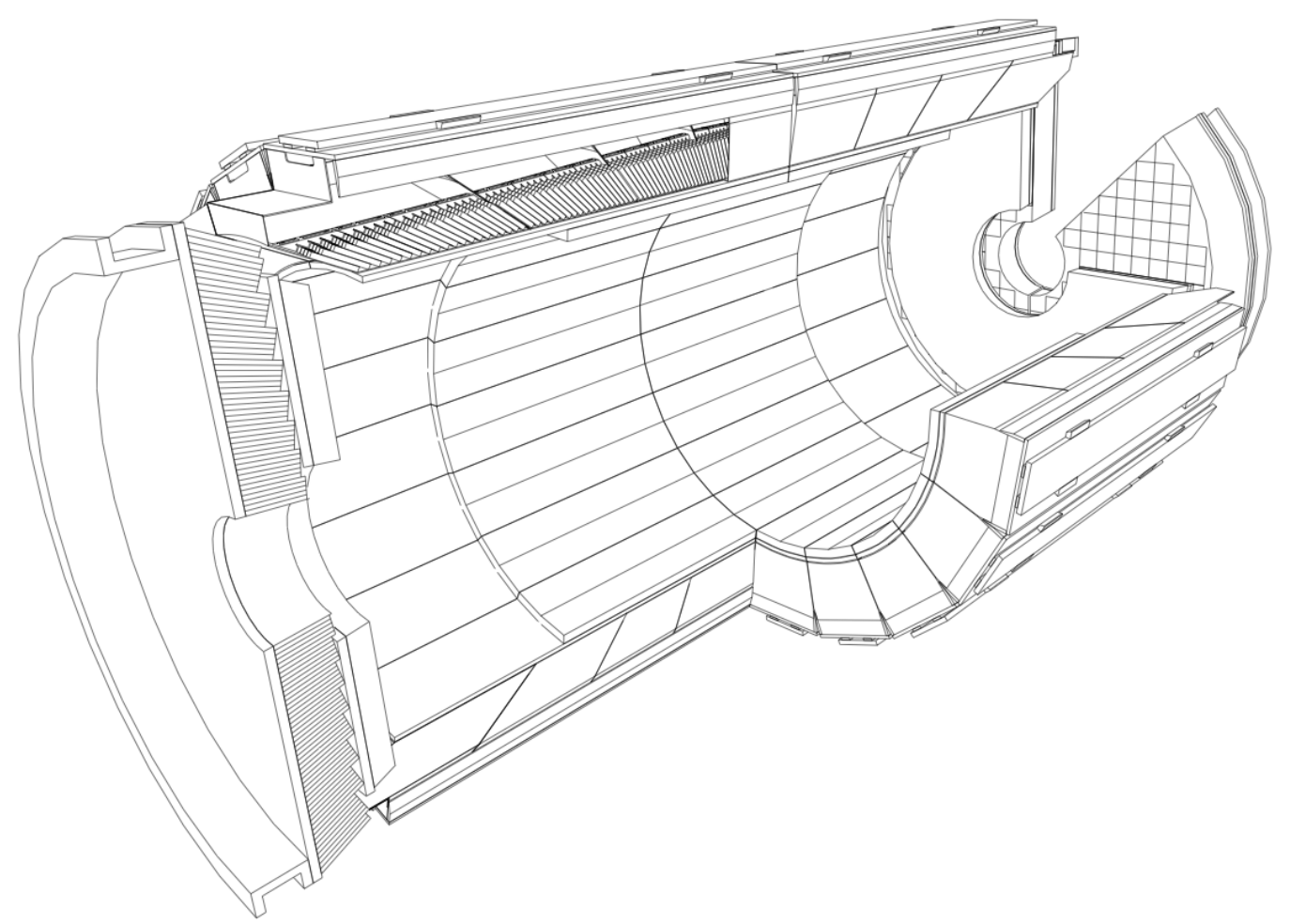

Figure 2.15: ECAL cutaway showing endcap (EE) and barrel (EB) subdetectors. [CERN]

The ECAL system contains $75,848 \mathrm{PbWO}_{4}$ crystals in order to have high 


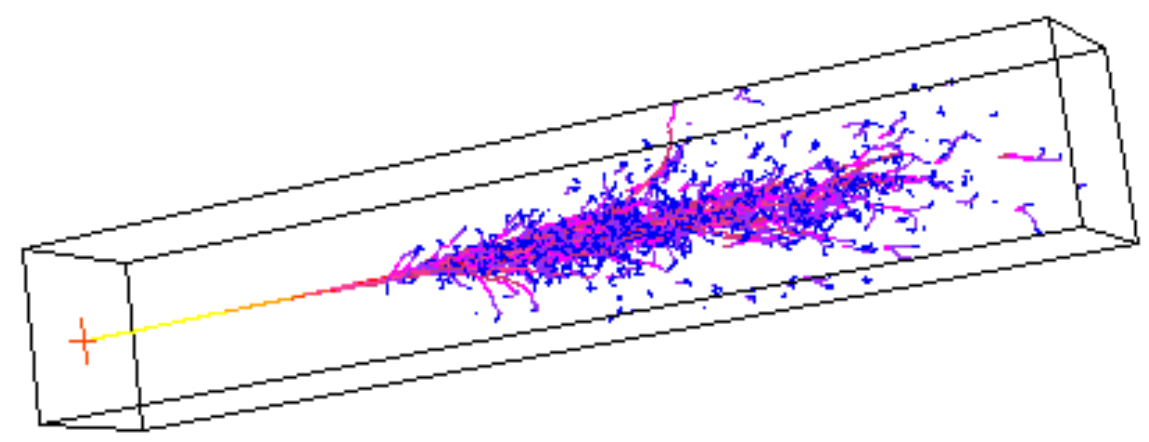

Figure 2.16: ECAL $\mathrm{PbWO}_{4}$ crystal shower simulation of a $24 \mathrm{GeV} e^{-}$. [CERN]

energy resolution and reconstruction ability. A charged particle passing into the crystal will cause a shower which in turn will cause the crystal to scintillate, depositing its light into the photodetector at the back of the crystal. See Figure 2.16 for a simulation of a $24 \mathrm{GeV}$ electron entering a crystal. 


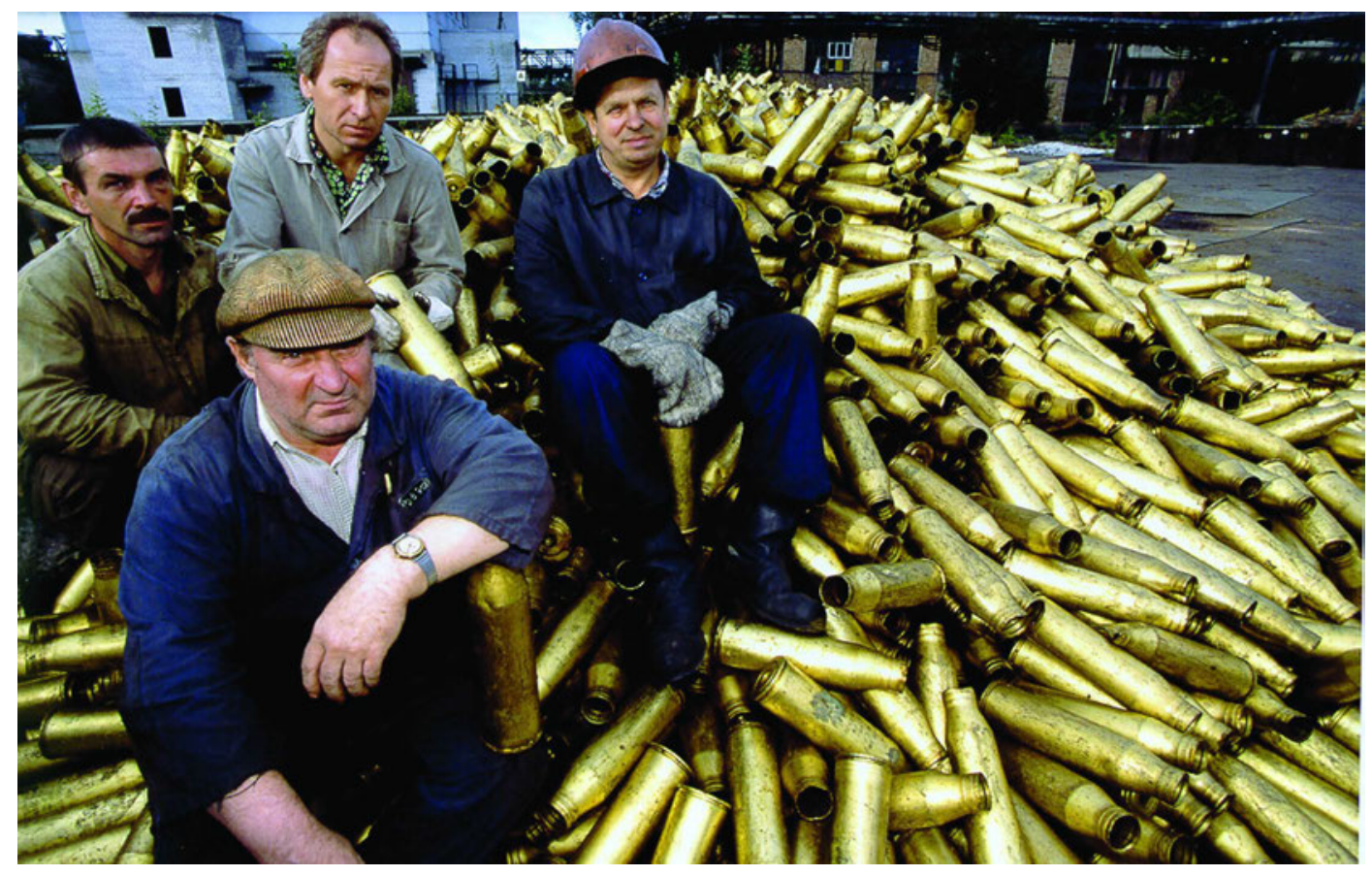

Figure 2.17: Russian Navy shells used to create the brass tiles of HCAL. Over one million brass shell casements were melted down for use in the HCAL detector. [CERN]

\subsubsection{Hadronic Calorimeter}

The HCAL system is composed of three main subsystems[24]:

HB: Hadronic Barrel $\eta<1.3$

HO: Hadronic Outer $\eta<1.3$

HE: Hadronic Endcap $1.3>\eta<3$

HF: Hadronic Forward $3>\eta<5.2$

Figure 2.17 is an image of a Russian crew responsible for decommissioning over one million Russian Navy brass shell casements leftover from WWII. The Russia 
and Dubna Member States (RDMS) collaboration was responsible for sourcing the material to be used in HCAL. Military grade brass turned out to have exactly the right properties, so an agreement was struck between the Russian Navy and CMS to turn over the more than one million shells ${ }^{7}$.

HCAL is a sampling calorimeter composed of brass wedges embedded with scintillating tiles. Wavelength shifting fibers are embedded in the scintillator tiles to carry the light generated in the tile by the particles to the hybrid photodiodes for signal readout. The plastic scintillator tiles will not withstand the radiation environment; replacement technology is being actively researched.

${ }^{7}$ This actually was not enough material to meet the 650 tons of brass required by HCAL. The remaining material was donated by the US, totalling over $\$ 1$ million USD. 


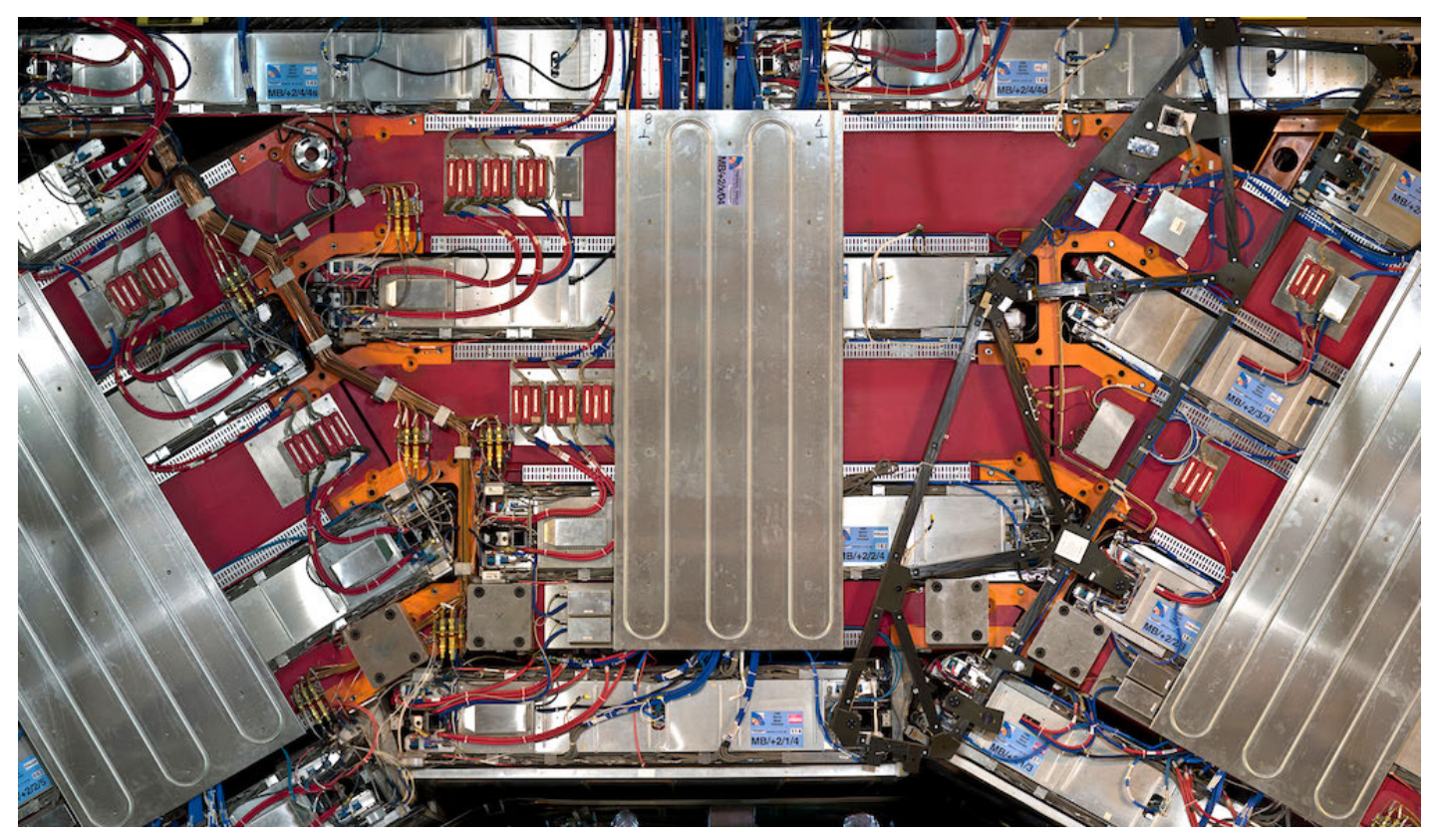

Figure 2.18: A segment of the CMS muon system. RPCs and DTs (silver) interspersed between the iron magnet return yokes (red). [CERN]

\subsubsection{Muon System}

A central feature of the CMS detector is the muon detector system; see Figure 2.18. The muon system can be divided into three main sub-units:

RPC: Resistive Plate Chambers

DT: Drift Tubes

CSC: Cathodic Strip Chambers

The RPCs and DTs are used in the barrel region of CMS, while the RPCs and CSCs are used in the end-cap region, see Figure 2.19. DTs cannot be used in the end-cap region due to the non-homogeneity of the magnetic field; see Figure 2.20. 


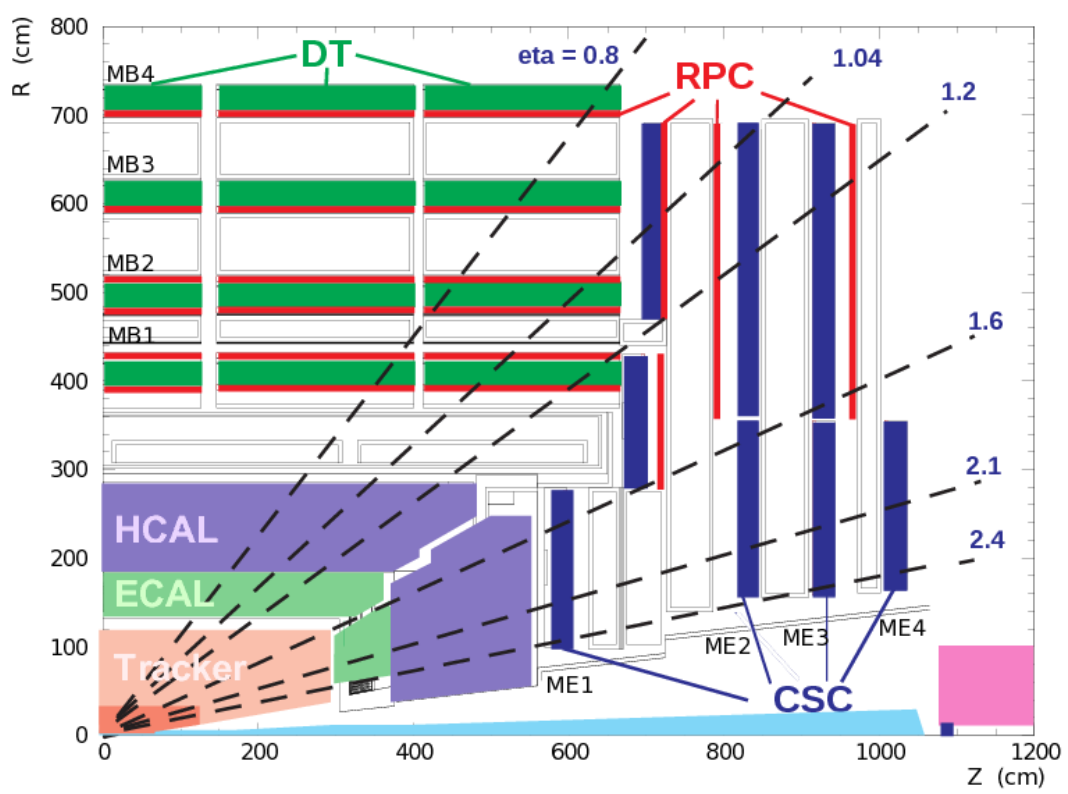

Figure 2.19: Longitudinal quadrant view of the CMS muon system. [CERN]

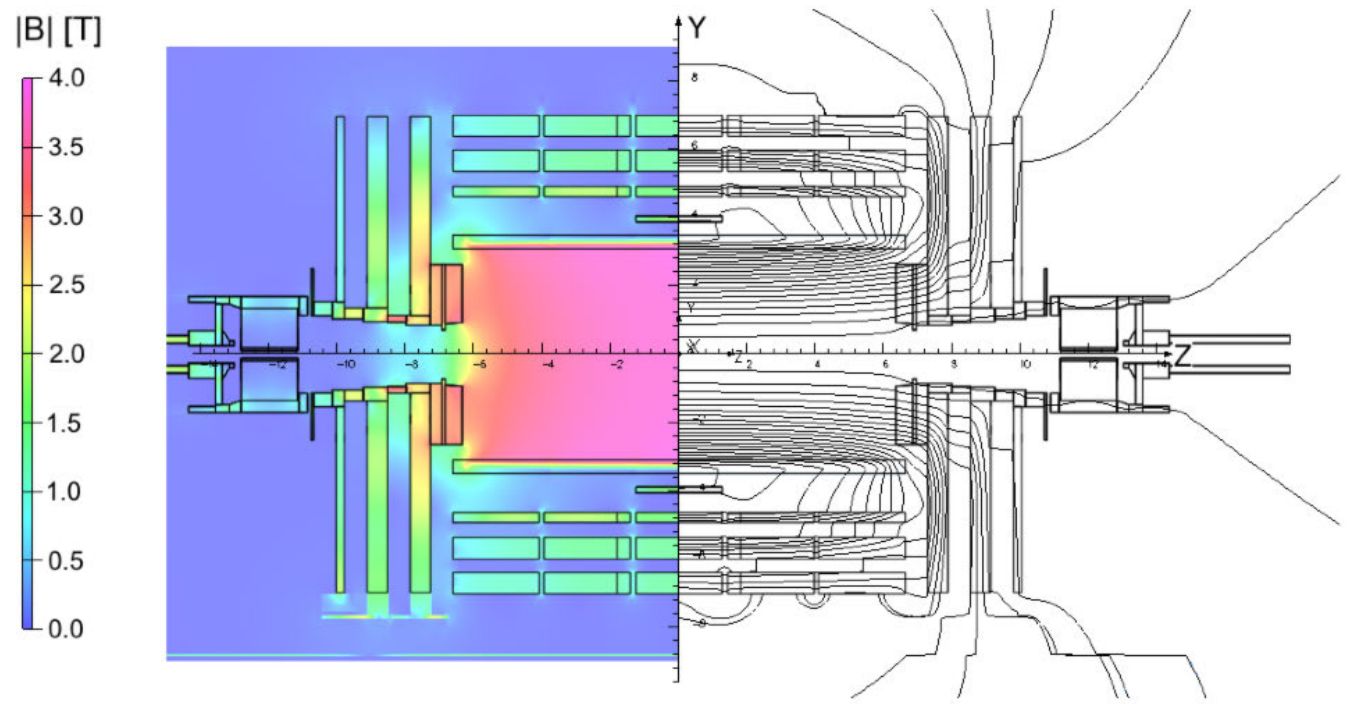

Figure 2.20: Simulated strength (left) and field lines (right) of the B-field in CMS. 


\subsubsection{Trigger}

The target event rate for recorded data in CMS is 100Hz. Every 25 ns more than 20 interactions take place. The electronics operate at a frequency of $\sim 10^{9} \mathrm{~Hz}$ and have about $\sim 10^{8}$ channels to be readout, creating raw data at a rate on the order of 100 TB every second. The CMS trigger system has the daunting task of reducing this data rate to $100 \mathrm{MB} / \mathrm{s}$ without discarding meaningful events. This job is left to the Level 1 (L1) Trigger and the High Level Trigger (HLT).

The HLT can only handle a data input rate of $100 \mathrm{kHz}$, requiring the L1 trigger to reduce the raw detector data rate by a factor of 10,000. The L1 trigger processes an event every $3.2 \mu \mathrm{s}$, requiring the detector to store a buffer of events totaling 128 bunch crossings. The L1 trigger makes its decisions taking input from the calorimeter trigger, the muon trigger, the global trigger, and the TTC (timing, trigger, and control system).

The HLT has roughly 1 second to make a final decision on an event passed by the L1 trigger, and can therefore incorporate the full calorimeter granularity and tracker system into the decision making process. The goal here is to reduce the event rate further by a factor of 1,000 .

\subsubsection{CMS Software}

This section describes the software tools used for the studies contained within this thesis. The CMS Experiment has developed a coherent set of data simulation, processing, and analysis software known as CMS Software, or CMSSW. CMSSW is 
a modular framework written almost entirely in $\mathrm{C}++$ and Python. CMSSW was developed entirely alongside the ROOT analysis framework. ROOT is an object oriented analysis package utilizing objects to contain data. This makes the task of handling CMS data much simpler and more powerful.

The backbone of the CMSSW system is a class-based architecture known as The Event Data Model, or EDM. Central to CMSSW is the idea of an Event in CMS, in both the literal and virtual sense. For example, a real muon reconstructed in CMS is referred to as a physics object in both the muon software class and in the actual detector.

Information in an event can only be manipulated independently by discrete modules, where most often the modules need to be executed on the data in a particular order, as the necessary data formats may not yet exist in the event. The various modules can be grouped into six separate categories, as follows:

Source: The source modules most generally import RAW data from the detector in the form of a ROOT file in the ROOT data format, though it is modular enough to take in custom data formats. In the case of $\mathrm{MC}$ generation, the source is used as a container to hold the generated events before being processed by other modules.

EDProducer: RAW data from CMS is generally not very useful to physicists doing analysis, so EDProducers are used to produce Event Data. Example packages would turn raw hits from the Tracker into a collection of reconstructed tracks, which could be used by another EDProducer module to reconstruct an electron 
from the tracks. In this case, the causal nature of the EDProducers is apparent.

EDFilter: Often times a user will want to remove an event or kill a process that is not useful. The EDFilter reads a single event and returns a boolean that is used by the framework to determine whether or not to continue the process. An example is the process StopAfterNEvents, which will kill the process path after reaching the critical number of events set by the user. Another, NTrackFilter, might kill the event if too many tracks are reconstructed.

EDAnalyzer: Once the data has been processed and the preferred data formats obtained, a physicist will want to analyze the events without adding or removing information to the event. An EDAnalyzer module is used to analyze events without altering them, and is typically used to create output histograms in the ROOT format, although any format can be used if programmed by the user.

EDLooper: Occasionally a physicist will find themselves wanting to tune some parameters and re-processing the source multiple times. The EDLooper is used for this.

OutputModule: After all analysis is done, an OutputModule is used to write the data from an event to an external source. Most commonly, PoolOutputModule is used to write the output data to a ROOT file.

All object classes and data formats are defined within a given CMSSW release, with versions ranging from CMSSW_1_1_1 up to CMSSW_7_0_0 and beyond, with custom releases for studying specific features or testing upgrades of CMS routinely 
developed and maintained. CMSSW is housed in a git repository on GitHub[25]. The analysis in this thesis uses CMSSW_5_3_8.

\subsubsection{CMSSW and CMS Simulation}

The entire CMS detector has been modeled in the software package GEANT4 and interfaced with CMSSW. There are two main detector simulation and modeling packages:

- Full Simulation

- Fast Simulation

Full simulation is termed fullSim and encodes every aspect of CMS into GEANT4, from cabling, detector materials, to detector component efficiencies and noise simulations. This can make for extremely long simulation run-times. As a particle is stepped through the detector, each property is simulated, from its interaction with the detector, to possible showering, and the resulting detector response. A single event can take as much as 20 minutes to be modeled. Considering that a minimum event set is generally required to be on the order of five thousand events, it can take more than 24 hours to generate a dataset of this size.

Fast simulation is nicknamed fastSim and is a parameterization of detector characteristics based on several runs of the more realistic fullSim, test beam results, and other studies. The advantage is lower run times for simulations while maintaining real detector responses. Instead of simulating the detector response with every step a particle takes, a parameterization is used that can range from a lookup table, to a 
functional, or an ntuple.

\subsection{Particle Identification}

The CMS Particle Object Groups (POG) are responsible for determining how the detector signals from the previously described detector elements should be reconstructed into real physics objects to be used by physicists in their analysis. The following describes the processes involved with determining the particles in the final state of the search described in Chapter 3.

\subsubsection{Muon Reconstruction}

There are three main methods to reconstruct muons. The first uses the CMS tracker, referred to as tracker muons. The second uses the main muon subsystem consisting of the RPCs, DTs, and CSCs, referred to as standalone muons. The third and most complete method combines the information from the tracker system and the muon system.

Tracker Muons: Track seeds produced in the PIXEL detector are propagated to the rest of the detector volume by connecting the tracks to form a trajectory.

Standalone Muons: Muon hits in the individual layers of RPCs and DTs or CSCs are stitched together to form trajectories.

Global Muons: A global fit is applied to the trajectory created between hits in the tracker system and the muon system. 
The equations of motion for a charged particle (like the muon) through CMS is written as:

$$
\frac{d^{2} \vec{r}}{d s^{2}}=\frac{q}{p} \frac{d \vec{r}}{d s} \times \vec{B}(\vec{r})
$$

Given that $\vec{B}=B \hat{z}$ in CMS and choosing $d s^{2}=d x^{2}+d y^{2}+d z^{2}$, the equations of motion can be written as:

$$
\begin{gathered}
x(s)=x_{0}+R_{H}\left[\cos \left(\phi_{0}+h \cdot s \cdot \cos \left(\lambda / R_{H}\right)-\cos \left(\phi_{0}\right)\right)\right] \\
y(s)=y_{0}+R_{H}\left[\sin \left(\phi_{0}+h \cdot s \cdot \cos \left(\lambda / R_{H}\right)-\sin \left(\phi_{0}\right)\right)\right] \\
z(s)=z_{0}+s \cdot \sin (\lambda) \\
R_{H}=\frac{p \cdot \cos (\lambda)}{q B} \\
\lambda=\arcsin \left(\frac{d z}{d s}\right)
\end{gathered}
$$

where $R_{H}$ is the helix radius, $\phi_{0}$ is the azimuthal angle with respect to the helix axis, and $\lambda$ is the slope angle.

This general method is applied in each muon reconstruction algorithm, along with the Kalman filter extrapolation procedure [26] which matches a set of hits in one layer to hits in a neighboring layer with algorithms that account for uncertainties (like position resolution and detector resolution) and multiple scattering.

This analysis requires muons to both be global muons and satisfy so-called particle-flow ID requirements, explained in Section 2.4.5.

\subsubsection{Jet Reconstruction}

Jets are complex objects that show up in the detector as large deposits of energy in the calorimeters, resulting from the hadronization of hard parton scattering, 
even including photons and electrons. It is therefore important for the CMS software to do a good job in reconstructing and categorizing the jet objects in the CMS detector.

There are several algorithms used to tag jets; this analysis uses the anti- $k_{T}$ algorithm. Each algorithm uses a combination of hits in cells from ECAL and HCAL in neighboring $(\eta, \phi)$ space, referred to as calorimeter towers. How the towers are clustered together to form jets depends on the algorithm used.

The anti- $k_{T}$ algorithm imagines a jet as a cone shape originating from the interaction point. The two jets chosen for the search described in Section 3 require a cone with an angular radius $\Delta R=\sqrt{(\Delta \eta)^{2}+\left(\Delta \phi^{2}\right)}$ of 0.5 .

The anti- $k_{T}$ algorithm combines two other general jet clustering algorithms, ones based on iterative cone models and others based on sequential recombination $\left(k_{T}\right.$, Cambridge/Aachen).

\subsubsection{Jet Energy and Resolution Scales}

Calorimeters do not respond linearly to particles as a function of their energy, making it a challenge to translate the measured energy in the detector to the true particle energy. In CMSSW, several tools are available to apply corrections to the jet energies, developed by the Jet Energy Resolution and Corrections Subgroup (JERC).

The two main corrections used in this analysis apply corrections to the jet energy scale (JES) and the jet energy resolution (JER). The JES can be thought of as the accuracy of the jet energy reconstruction, whereas the JER can be thought 
of as the precision of the jet energy reconstruction. Reference [27] goes into detail on how the jet energy corrections are calculated in CMS. The JERC maintains an up-to-date list of corrections and data/MC scale factors for use in CMS analyses[28].

\subsection{4 b-Tag Jets}

Jets created from $b$-quarks are tagged in CMS with some very clever algorithms, each taking advantage of the b-quark's unusually long lifetime $\left(\sim 10^{-12} \mathrm{~s}\right)$. The tagging methods generally involve reconstructing the displaced vertices between the original collision vertex and the vertex of the jet created from the decay of the b-quark.

This analysis discriminates b-tag jets using the Combined Secondary Vertex (CSV) medium working point, as defined by the b-tag POG[29].

\subsubsection{Particle Flow}

CMS physicists have implemented a method known as particle flow (PF) that attempts to combine the information from all detector elements in order to reconstruct all detectable types of particles: electrons, muons, photons, and jets. Prior to $\mathrm{PF}$, each type of particle was reconstructed using their own individual algorithms. Particle flow is a global algorithm designed to reconstruction particles using information from each CMS system.

After the tracking algorithms and clustering algorithms are finished, the PF link algorithm iterates through the tracks to compare with clusters in the calorimeters and hits in the muon system. If there is overlap, various filters are applied to ensure 
quality particle reconstruction.

The analysis described in Section 3 uses PF ID for all physics objects. 


\section{CHAPTER 3 \\ A SEARCH FOR A HEAVY MAJORANA NEUTRINO}

This search involves the cutting edge physics surrounding neutrino mass. For a thorough review of neutrino physics, the reader should consult [30, 31, 32].

\subsection{Introduction}

One of the first hints at physics beyond the Standard Model (BTSM) is the fact that neutrinos have non-zero mass $[33,34]$. The Standard Model cannot be used to explain neutrino mass, implying a lack of understanding by physicists of the mechanisms underlying mass generation for fundamental particles.

The leading theoretical extension to the Standard Model which explains neutrino mass is the so-called "seesaw" mechanism[35, 36, 37, 38]. The seesaw mechanism posits that the smallness of the observed neutrino masses is due to the largeness of the mass of a new heavy state, $N$. The neutrino mass, $m_{\nu}$, can be written in terms of the heavy Majorana neutrino $(\mathrm{HMN})$ mass $M_{N}: m_{\nu} \sim y_{\nu}^{2} v^{2} / M_{N}$, where $y_{\nu}$ is a Yukawa coupling and $v$ is the Higgs vacuum expectation value in the standard model. Due to the new heavy neutrino's Majorana nature it is its own anti-particle, which allows for processes that violate lepton number conservation by two units. As the leading candidate for explaining the smallness of the neutrino masses, experimentally searching for Majorana neutrinos is thus of fundamental importance.

The production of HMNs at hadron colliders has been studied fairly extensively $[39,40,41,42,43]$, with interest renewed by the inauguration of the Large Hadron 
Collider $[44,45,46]$.

This search, as well as those at previous colliders (LEP, LHC2011), uses a model-independent phenomenological approach, assuming that the HMN mass, $M_{N}$, and $V_{\ell N}$, (the mixing element describing the mixing between the heavy Majorana neutrino and the SM neutrino $\nu_{\ell}$ of flavor $\ell$ ), are free parameters.

Previous direct searches for heavy Majorana neutrinos have been reported by the DELPHI[47] and L3[48] collaborations. These searches set limits on the mixing element squared ${ }^{1}$ for Majorana neutrino masses below $\sim 90 \mathrm{GeV} / c^{2}$. More recently, the CMS[49] and ATLAS Collaborations [50] have performed direct searches and set limits on $\left|V_{e N}\right|^{2}$ for masses up to $\sim 200 \mathrm{GeV} / c^{2}$ and on $\left|V_{\mu N}\right|^{2}$ for masses up to $\sim 300$ $\mathrm{GeV} / c^{2}$.

The analysis described in this thesis is a detailed search for the resonance production of the HMN at the LHC with the CMS detector using the 2012 data set with an integrated luminosity of $19.7 \mathrm{fb}^{-1}$. The search is for events with same sign muons and two jets in the final state. The Feynman diagram for this process is shown in Figure 3.1. An important motivation for this search is that Standard Model processes creating this signature are very small, as will be detailed in Section 3.6.

That being said, there are still Standard Model processes that can contribute to what is called 'background' that have to be understood, including so-called "fake muon" processes in which muons that aren't a product of the signal process might be

\footnotetext{
${ }^{1}$ The mixing element squared is directly proportional to the HMN production cross section.
} 
mistaken for muons that are.

Throughout this analysis, muons are referred to as either 'prompt', or 'nonprompt'. Prompt muons are muons that can be traced back to the primary vertex, (where the collision took place), and satisfy all of our muon selection criteria. Generally, prompt muons originate from the decay of a $\mathrm{W}$ or $\mathrm{Z}$ boson, or the hypothesized HMN signal. The largest prompt contamination background is from Standard Model diboson production $(W W, Z Z)$, where the bosons decay directly to muons.

Non-prompt muons are muons that originate from jets, and can be real muons generated via the decay of a b-quark or so-called decays-in-flight, or jets mis-identified as a muon, each with the potential to contaminate the signal region.

Figure 3.2 shows the cross section for HMN production and decay via the process in Figure 3.1 as a function of Majorana neutrino mass for $\left|V_{\ell N}\right|^{2}=1.0$. The plot shows the results at leading order from del Aguila et al. [45] for $\sqrt{s}=7 \mathrm{TeV}$ and $8 \mathrm{TeV}$.

This analysis has 5 parts: Designing selection criteria to maximize signal and minimize background; estimating backgrounds; validating selection and background on control datasets; comparing predictions with data; and interpreting results.

\subsection{Monte Carlo Event Generation}

Monte Carlo (MC) event generators have been developed to assist physicists in designing analyses by simulating the decays and kinematics of a physical process, as well as how the signal they are searching for may show up in the real CMS detector. 


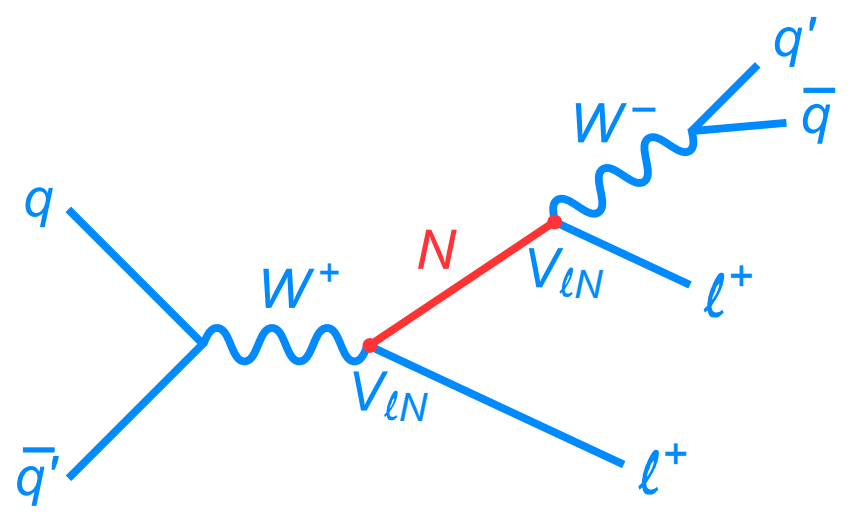

Figure 3.1: A Feynman diagram for resonance production of a Majorana neutrino $(\mathrm{N})$ in hadron colliders.

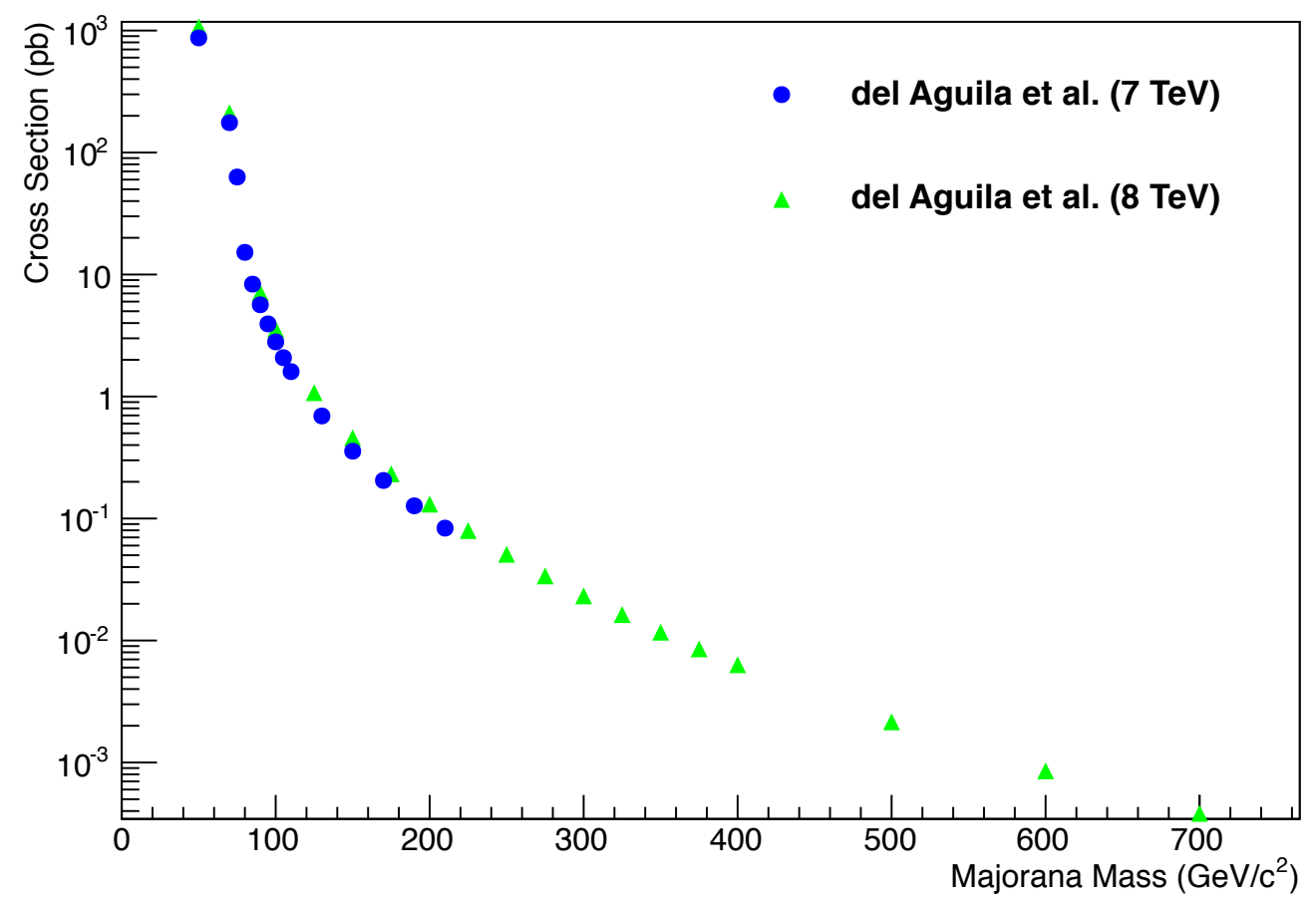

Figure 3.2: Cross sections for resonance production of a Majorana neutrino as a function of Majorana neutrino mass. [51] 
Two groups of MC datasets were used in this analysis: one group for signal, the other for background. The datasets used for background studies are referred to as Standard Model MC samples, whereas signal MC samples are referred to as, simply, signal datasets.

There are two steps to the generation process:

- Generation of the event and its decay process

- Simulation of the detector response

The three main software packages used by this analysis to generate, hadronize, and simulate the signal and background MC datasets are the ALPGEN, Pythia, and GEANT4 software packages, described below.

\subsubsection{Signal Event Generation}

Event generation for the HMN signal was done using the software package ALPGEN [52]. The production and decay process was implemented in ALPGEN to leading order (LO) as described in [45]. The produced datasets were simulated at a collision energy of $8 \mathrm{TeV}$, with the parton distribution function (PDF) set CTEQ6M used. The output was stored in Les Houches 1.0 format, (see Reference [53] for a description of the format), and supplied to the CMS Data Operations group for official MC production.

After generation, the events are hadronized using Pythia[54] version 6.4 to generate events for each HMN mass. Once the events are generated, they must be simulated along with detector quirks like electronic noise or gaps in coverage in order 
to understand how the particles will be reconstructed by the detector.

The official production signal MC samples along with their LO cross sections as a function of HMN mass are shown in Table 3.2, with abbreviations used listed in Table 3.3. These samples were generated using an HMN-muon coupling value of $S_{\mu \mu}=\left|V_{\mu N}\right|^{2}=1$

This does not include next-to-leading order (NLO) calculations, though a multiplicative $k$-factor of 1.34 is applied to account for higher order corrections. This is based on the $k$-factors calculated for $W^{\prime}$ production with the NNLO calculation as in FEWZ $^{2}[55,56,57]$.

\subsubsection{Simulation}

Detector simulation of the generated events occurs after the generated events are interfaced with a specific CMSSW version, as each CMSSW version differs in how it simulates the detector response. The generated event datasets for this analysis were interfaced with CMSSW_5_3_12_patch2, where parton showering, vertex smearing, GEANT4[58] detector simulation, digitization of the millions of simulated electronics signals, and particle reconstruction were performed. The MC samples are reweighted to ensure the proper simulation of the number of interactions per beam crossing, based on previous LHC operation history.

An entire software model of the CMS detector was developed using GEANT4 in order to effectively simulate the CMS detector response. Referred to as "Full

\footnotetext{
${ }^{2} \mathrm{FEWZ}$ is a software package for hadronic Z production, short for Fully Exclusive $W, Z$ Production through $N N L O$ in $p Q C D$.
} 
Simulation" (FullSim), each particle in an event is simulated step by step through the detector, a process called tracking, (not to be confused with the tracker subdetector). Each step considers a number of detector properties including the physics of the decay process and the generation of secondary particles radiated by the effects of the magnetic field on the particles, or showers created by interactions of the particle with detector materials.

\subsubsection{Standard Model Monte Carlo Samples}

Monte Carlo datasets produced for background estimation are listed in Table 3.1, with the abbreviations used listed in Table 3.3. The Standard Model backgrounds included were diboson production, $t \bar{t} W$ production, same sign $W$ production via double $W$-strahlung and double parton scattering. The $t \bar{t}, W+$ jets, and QCD samples are used to validate the 'fake background' estimation method, described in Section 3.6. Each sample is reweighted to ensure proper simulation of the number of interactions per crossing due to pileup.

A list of the signal MC datasets used in this analysis can be found in Table 3.2, with the abbreviations used in the table found in Table 3.3.

\subsection{LHC Collision Datasets}

Data from LHC proton-proton collisions recorded using the CMS detector during the 2012 run was used in this analysis. 
Table 3.1: Summary of Standard Model Monte Carlo datasets used.

\begin{tabular}{|c|c|c|c|}
\hline Dataset & Dataset Name & $\sigma(p b)$ & $\mathcal{L} d t\left(\mathrm{pb}^{-1}\right)$ \\
\hline$W^{+} W^{-}$ & /WW_TZ_8TeV_pythia6_TA/S12a & 54.8 & $1.82 \times 10^{5}$ \\
\hline$W Z$ & /WZ_TZ_8TeV_pythia6_TA/S12a & 33.2 & $3.01 \times 10^{5}$ \\
\hline$Z Z$ & /ZZ_TZ_8TeV_pythia6_TA/S12a & 17.7 & $5.55 \times 10^{5}$ \\
\hline$W \gamma$ & /WGToLNuG_TZ-MG-TA/S12a & 462 & $1.04 \times 10^{4}$ \\
\hline$W^{+} W^{+}$ & /WpWpqq_8TeV-MG/S12a & 0.248 & $4.03 \times 10^{5}$ \\
\hline$W^{-} W^{-}$ & /WmWmqq_8TeV-MG/S12a & 0.0888 & $1.08 \times 10^{6}$ \\
\hline $\operatorname{dp} W^{ \pm} W^{ \pm}$ & /WW_DoubleScattering_8TeV-PY/S12a & 0.588 & $1.42 \times 10^{6}$ \\
\hline$W W \gamma$ & /WWGJets_8TeV-MG_v2/S12a & 0.528 & $5.76 \times 10^{5}$ \\
\hline$W W W$ & /WWWJets_8TeV-MG/S12a & 0.082 & $2.68 \times 10^{6}$ \\
\hline$W W Z$ & /WWZNoGstarJets_8TeV-MG/S12a & 0.058 & $3.83 \times 10^{6}$ \\
\hline$W Z Z$ & /WZZNoGstarJets_8TeV-MG/S12b & 0.0197 & $1.12 \times 10^{7}$ \\
\hline$Z Z Z$ & /ZZZNoGstarJets_8TeV-MG/S12a & $5.53 \times 10^{-3}$ & $4.07 \times 10^{7}$ \\
\hline$t \bar{t}$ & /TTJets_MBD_TZ_8TeV-MG-TA/S12b & 234 & $2.96 \times 10^{4}$ \\
\hline$t\left(t W^{-}\right)$ & /T_tW-CH-DR_TZ-powheg-TA/S12a & 10.7 & $4.65 \times 10^{4}$ \\
\hline $\bar{t}\left(\bar{t} W^{+}\right)$ & /Tbar_tW-CH-DR_TZ-powheg-TA/S12a & 10.7 & $4.61 \times 10^{4}$ \\
\hline$t(s-\mathrm{CH})$ & /T_s-CH_TZ-powheg-TA/S12a & 2.82 & $9.22 \times 10^{4}$ \\
\hline $\bar{t}(s-\mathrm{CH})$ & /Tbar_s-CH_TZ-powheg-TA/S12a & 1.57 & $8.92 \times 10^{4}$ \\
\hline$t(t-\mathrm{CH})$ & $/ \mathrm{T}_{-} \mathrm{t}-\mathrm{CH}_{-} \mathrm{TZ}-\mathrm{powheg}-\mathrm{TA} / \mathrm{S} 12 \mathrm{a}$ & 47.0 & $8.00 \times 10^{4}$ \\
\hline $\bar{t}(t-\mathrm{CH})$ & /Tbar_t-CH_TZ-powheg-TA/S12a & 25.0 & $7.74 \times 10^{4}$ \\
\hline $\bar{t} \bar{t} W$ & /TTWJets_8TeV-MG/S12a & 0.232 & $8.45 \times 10^{5}$ \\
\hline$t \bar{t} Z$ & /TTZJets_8TeV-MG_v2/S12a & 0.174 & $1.21 \times 10^{6}$ \\
\hline$t \bar{t} W W$ & /TTWWJets_8TeV-MG/S12a & $2.04 \times 10^{-3}$ & $1.07 \times 10^{8}$ \\
\hline Drell-Yan & /DYJetsToLL_M-10To50_TZ-MG/S12a & $1.11 \times 10^{4}$ & $3.42 \times 10^{3}$ \\
\hline Drell-Yan & /DYJetsToLL_M-50_TZ-MG-TB/S12a & $3.50 \times 10^{3}$ & $8.69 \times 10^{3}$ \\
\hline$W+$ jets & /WJetsToLNu_TZ-MG-TB/S12c & $3.75 \times 10^{4}$ & $1.54 \times 10^{3}$ \\
\hline QCD_mu15 & /QCD_Pt_20_MuE_15_TZ_PY/S12d & $3.64 \times 10^{8}$ & 160 \\
\hline QCD_mumu & /QCD2MuMu_2MupPEFF_8TeV-PY/S12a & $4.96 \times 10^{10}$ & 54.2 \\
\hline QCD_mu5 & /QCD_Pt-15to20_MuE5_TZ_PY/S12c & $7.02 \times 10^{8}$ & $6.29 \times 10^{-1}$ \\
\hline QCD_mu5 & /QCD_Pt-20to30_MuE5_TZ_PY/S12a & $2.87 \times 10^{8}$ & 4.55 \\
\hline QCD_mu5 & /QCD_Pt-30to50_MuE5_TZ_PY/S12a & $6.61 \times 10^{7}$ & 11.9 \\
\hline QCD_mu5 & /QCD_Pt-50to80_MuE5_TZ_PY/S12a & $8.08 \times 10^{6}$ & 58.8 \\
\hline QCD_mu5 & /QCD_Pt-80to120_MuE5_TZ_PY/S12a & $1.03 \times 10^{6}$ & 228 \\
\hline QCD_mu5 & /QCD_Pt-120to170_MuE5_TZ_PY/S12a & $1.58 \times 10^{5}$ & $1.14 \times 10^{3}$ \\
\hline QCD_mu5 & /QCD_Pt-170to300_MuE5_TZ_PY/S12a & $3.40 \times 10^{4}$ & $3.3 \times 10^{3}$ \\
\hline QCD_mu5 & /QCD_Pt-300to470_MuE5_TZ_PY/S12a & $1.76 \times 10^{3}$ & $5.16 \times 10^{4}$ \\
\hline QCD_mu5 & /QCD_Pt-470to600_MuE_TZ_PY/S12a & 115 & $3.21 \times 10^{5}$ \\
\hline QCD_mu5 & /QCD_Pt-600to800_MuE5_TZ_PY/S12a & 27.0 & $1.53 \times 10^{6}$ \\
\hline QCD_mu5 & /QCD_Pt-800to1000_MuE5_TZ_PY/S12a & 3.57 & $1.11 \times 10^{7}$ \\
\hline QCD_mu5 & /QCD_Pt-1000_MuE5_TZ_PY/S12a & 0.774 & $4.56 \times 10^{7}$ \\
\hline
\end{tabular}

Note: Abbreviations used are listed in Table 3.3 
Table 3.2: Majorana neutrino signal MC samples generated with $\left|V_{\mu N}\right|^{2}=1$.

\begin{tabular}{llcc}
\hline Mass $\left(\mathrm{GeV} / \mathrm{c}^{2}\right)$ & Dataset Name & $\sigma(\mathrm{pb})$ & $\int \mathcal{L} d t\left(\mathrm{pb}^{-1}\right)$ \\
\hline 40 & /MN_M-40_TZ-alpgen/S12a & $1515 \pm 1$ & 65.6 \\
50 & /MN_M-50_TZ-alpgen/S12 & $1071 \pm 1.4$ & 46.68 \\
60 & /MN_M-60_TZ-alpgen/S12a & $607.6 \pm 0.4$ & 163.7 \\
70 & /MN_M-70_TZ-alpgen/S12 & $212.0 \pm 0.3$ & 235.9 \\
80 & /MN_M-80_TZ-alpgen/S12a & $19.06 \pm 0.01$ & 5165 \\
90 & /MN_M-90_TZ-alpgen/S12 & $(7105 \pm 3) \times 10^{-3}$ & 7037 \\
100 & /MN_M-100_TZ-alpgen/S12 & $(3562 \pm 1) \times 10^{-3}$ & 14037 \\
125 & /MN_M-125_TZ-alpgen/S12 & $(1076.7 \pm 0.3) \times 10^{-3}$ & 46242 \\
150 & /MN_M-150_TZ-alpgen/S12 & $(4594 \pm 1) \times 10^{-4}$ & 108827 \\
175 & /MN_M-175_TZ-alpgen/S12 & $(2326.6 \pm 0.7) \times 10^{-4}$ & 214442 \\
200 & /MN_M-200_TZ-alpgen/S12 & $(1312.7 \pm 0.4) \times 10^{-4}$ & 380833 \\
225 & /MN_M-225_TZ-alpgen/S12 & $(7966 \pm 2) \times 10^{-5}$ & 627547 \\
250 & /MN_M-250_TZ-alpgen/S12 & $(5093 \pm 2) \times 10^{-5}$ & 979699 \\
275 & /MN_M-275_TZ-alpgen/S12 & $(3386 \pm 1) \times 10^{-5}$ & 1476652 \\
300 & /MN_M-300_TZ-alpgen/S12 & $(2321.4 \pm 0.7) \times 10^{-5}$ & 2153700 \\
325 & /MN_M-325_TZ-alpgen/S12 & $(1631.7 \pm 0.5) \times 10^{-5}$ & 3063798 \\
350 & /MN_M-350_TZ-alpgen/S12 & $(1170.5 \pm 0.4) \times 10^{-5}$ & 4271252 \\
375 & /MN_M-375_TZ-alpgen/S12 & $(8545 \pm 3) \times 10^{-6}$ & 5826500 \\
400 & /MN_M-400_TZ-alpgen/S12 & $(6332 \pm 2) \times 10^{-6}$ & 7879057 \\
500 & /MN_M-500_TZ-alpgen/S12 & $(2154 \pm 1) \times 10^{-6}$ & 23208152 \\
\hline
\end{tabular}


Table 3.3: Abbreviations used in Tables 3.1 and 3.2

\begin{tabular}{cc}
\hline Abbreviation & Full Form \\
\hline S12 & Summer12_DR53X-PU_S10_START53_V19-v1/AODSIM \\
S12a & Summer12_DR53X-PU_S10_START53_V7A-v1/A0DSIM \\
S12b & Summer12_DR53X-PU_S10_START53_V7C-v1/AODSIM \\
S12c & Summer12_DR53X-PU_S10_START53_V7A-v2/AODSIM \\
S12d & Summer12_DR53X-PU_S10_START53_V7A-v3/AODSIM \\
dp & double parton \\
MuE & MuEnrichedPt \\
TZ & TuneZ2star_8TeV \\
MG & madgraph \\
PEFF & PtEtaFilterFilter \\
MBD & MassiveBinDECAY \\
CH & channel \\
PY & pythia6 \\
TA & tauola \\
TB & tarball \\
MN & MajoranaNeutrinoToMuMu \\
\hline
\end{tabular}

\subsubsection{Collision Data}

Each data run must be handled independently, accounting for differences in the detector operating conditions. In this analysis, the data used was collected during run periods A, B, C, and D. Both SingleMu and DoubleMu datasets were used for background studies, with only the DoubleMu used for the signal search. Table 3.4 lists the collision data used in the analysis. The SingleMu and DoubleMu terminology refers to the triggers used to select the event, covered in Section 3.4.1. The total integrated luminosity of these data sets is $19.7 \pm 0.5 \mathrm{fb}^{-1}$. 
Table 3.4: Summary of 2012 datasets used.

\begin{tabular}{ll}
\hline Dataset & Run Range \\
\hline /SingleMu/Run2012A-22Jan2013-v1/AOD & $190456-193621$ \\
/SingleMu/Run2012B-22Jan2013-v1/AOD & $193833-196531$ \\
/SingleMu/Run2012C-22Jan2013-v1/AOD & $198022-203742$ \\
/SingleMu/Run2012D-22Jan2013-v1/AOD & $203777-208686$ \\
/DoubleMu/Run2012A-22Jan2013-v1/A0D & $190456-193621$ \\
/DoubleMuParked/Run2012B-22Jan2013-v1/AOD & $193833-196531$ \\
/DoubleMuParked/Run2012C-22Jan2013-v1/AOD & $198022-203742$ \\
/DoubleMuParked/Run2012D-22Jan2013-v1/AOD & $203777-208686$ \\
\hline
\end{tabular}

\subsection{Event Selection}

As shown in the Feynman diagram (Figure 3.1) the signal signature is characterized by two leptons with an accompanying $W$ boson. Considering the Majorana nature of the neutrino being searched for, the leptons can either have the same charge (same-sign), or opposite charge (opposite-sign). This analysis concentrates on samesign muons. Same-sign dimuon events have very low Standard Model backgrounds. With the final event signature chosen, the $W$ boson decays to a quark anti-quark pair, leading to two jets, establishing a clean signature free of Standard Model neutrinos.

The following is an outline of the selection procedure used to determine which events are most likely to contain the HMN production signature, as well as the selection procedure used to estimate the background contamination.

Each object, (i.e. trigger, jet, muon), in the event has its own selection procedure and criteria used to define its quality. Each parameter can be adjusted depending on the needs of the analysis. It is important to understand and quantify how the 
detector will actually see the event.

\subsubsection{Trigger}

Of the two primary datasets considered (SingleMu and DoubleMu) the signal event selection used the HLT_Mu17_TkMu8 trigger. This is a dimuon trigger, (meaning it requires at least two muons in the event), with the further requirement that the leading muon must have a $\mathrm{p}_{T}$ of at least $17 \mathrm{GeV} / c$ and the trailing muon must have a $\mathrm{p}_{T}$ of at least $8 \mathrm{GeV} / c$. The efficiency of this trigger is discussed in Section 3.5.1. These triggers result in a dataset with a total integrated luminosity of $19.7 \mathrm{fb}^{-1}$.

In order to determine the background, a fake rate for muons mistaken for those from the HMN signal are estimated with events collected using single muon triggers. The following triggers were used for the fake rate calculation:

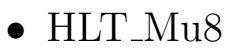

- HLT_Mu12

- HLT_Mu17

- HLT_Mu24

These triggers ${ }^{3}$ amount to a combined integrated luminosity of $123.4 \mathrm{pb}^{-1}$.

\subsubsection{Primary Vertex Selection}

The vertex of an event is the point in the detector where the daughter particle trajectories converge, i.e. the point of interaction. A well defined vertex creates the

\footnotetext{
${ }^{3}$ These triggers are prescaled, meaning only a small fraction of the triggered events are stored.
} 
possibility for the parent interactions of the reconstructed particles to be determined, thereby limiting the contamination of pileup or fakes. Every event was required to have at least one "good" vertex, characterized as meeting the following POG defined criteria:

- 4 fit degrees of freedom

- z coordinate $\left(d_{z}\right)$ within $24 \mathrm{~cm}$ of the detector center

- $\mathrm{x} / \mathrm{y}$ transverse coordinate $\left(d_{x y}\right)$ must be within $2 \mathrm{~cm}$ of the beam line

As a final requirement, the vertex with the largest summed squared- $\mathrm{p}_{T}$ is chosen as the primary vertex. This ensures that our final state products originate from the same interaction. While it is possible to get two muons and two jets flying through the detector from 4 separate interactions, they would not come from the HMN signal. By forcing the reconstructed particles to have originated from the same vertex, we can improve our confidence in selecting only the particles from a single interaction.

\subsubsection{Muon Selection}

The muon reconstruction and selection efficiency is one of the most important quantities in the analysis, and a good amount of time was spent deciding which selection criteria to use in order to optimize the signal and reduce the background.

An efficiency calculation analysis package was written to estimate muon selection efficiency as a function of every possible selection parameter. Each parameter was adjusted independently in order to understand how each selection affected the 
overall selection efficiency.

After two same-sign muons were determined to be in the event, the following selection criteria were further required of both muons:

- the candidate is a Global Muon and also satisfies particle-flow muon ID

- $\chi^{2} /$ number of degrees of freedom $<10$

- number of hits in the muon system $\geq 1$

- muon segments in at least two muon stations

- $d_{x y}$ relative to the primary vertex mentioned above $<0.05 \mathrm{~mm}$

- $d_{z}$ within $1 \mathrm{~mm}$ of primary vertex

- at leat one hit in pixel detector

- number of tracker layers with hits $>5$

- $\mathrm{p}_{T}>15 \mathrm{GeV} / \mathrm{c}$

- $|\eta|<2.4$

- isolated muon (where isolation values are all calculated in a $\Delta R$ cone of 0.3 which exclude the muon contribution):

$-\sum$ ECAL energy deposits $<4 \mathrm{GeV}$

$-\sum$ HCAL energy deposits $<6 \mathrm{GeV}$

- PF-based relative isolation $\left(\frac{E C A L_{\text {deposits }}+H C A L_{\text {deposits }}+\text { tracker }_{\text {deposits }}}{\mu_{p_{T}}}\right)<0.05$

These criteria were selected to ensure that two good muons that are well isolated are selected. Isolated means exactly what it sounds like: all by itself. This is determined by calculating the energy in the detector elements immediately surround- 
ing the muon's trajectory. If a muon was created in a jet, for example, the jet's energy would surround the muon, indicating to the physicist that this muon is not the muon this analysis is searching for.

\subsubsection{Jet Selection}

There is no upper limit placed on the number of jets in a selected event, though the two jets selected must be chosen carefully to ensure they are the best candidates to represent jets produced from the event signature. The HMN signature is one with two clean jets. Therefore, any events with a b-tagged jet (see Section 2.4.4) are excluded. "Lepton cleaning" is applied in order to veto any events which have an electron in them, and the jet selection algorithm coded for this analysis also vetoes any jets that contain a lepton.

The jets in the HMN signature with an HMN mass greater than $90 \mathrm{GeV} / c^{2}$ should originate from a $W$ boson. Therefore, the jet selection algorithm chooses the two jets, (called a dijet), which closest combine to give the $W$ mass for a signal mass greater than $90 \mathrm{GeV} / c^{2}$.

Prior to the dijet selection algorithm, a collection of jets must first be established, meeting, (or exceeding), the following POG defined criteria:

- corrected $\mathrm{p}_{T}>20 \mathrm{GeV} / c$

- $|\eta|<2.5$

- $\Delta R>0.4$ separation from any muon

- must be chosen by Particle Flow algorithm. (This suppresses jets from pileup.) 
- anti- $k_{T}$ algorithm with $R=0.5$

\subsubsection{Event Preselection}

This analysis separates the selection of events into two parts: preselection and full selection ${ }^{4}$.

After initially meeting the same-sign criteria and jet criteria described in Sections 3.4.3 and 3.4.4, several more criteria had to be met:

Muon $\mathbf{p}_{T}:{ }^{5}$ leading muon $\mathrm{p}_{T}>20 \mathrm{GeV} / c$.

Dimuon Mass: ${ }^{6}$ dimuon mass $>10 \mathrm{GeV} / c^{2}$.

Muon Veto: events with 3 or more muons are vetoed.

Electron Veto: events with an electron are vetoed.

This set of selection criteria comprise the preselection cuts.

Figures 3.3 and 3.4 show the invariant mass distributions for the dimuon mass and mass of the $(\mu \mu \mathrm{jj})$ of events passing preselection, including both data and Monte Carlo background samples.

\footnotetext{
${ }^{4}$ Full selection comprises cuts that were optimized based on the preselection criteria, and is motivated in the following sections.

${ }^{5}$ Even though the trigger used is the HLT_Mu17_Mu8, corresponding to a leading muon with $\mathrm{p}_{T}>17 \mathrm{GeV} / c$, the trigger is not fully efficient until $20 \mathrm{GeV} / c$.

${ }^{6}$ The dimuon mass requirement limits contamination from low mass resonances without affecting the HMN signal efficiency.
} 


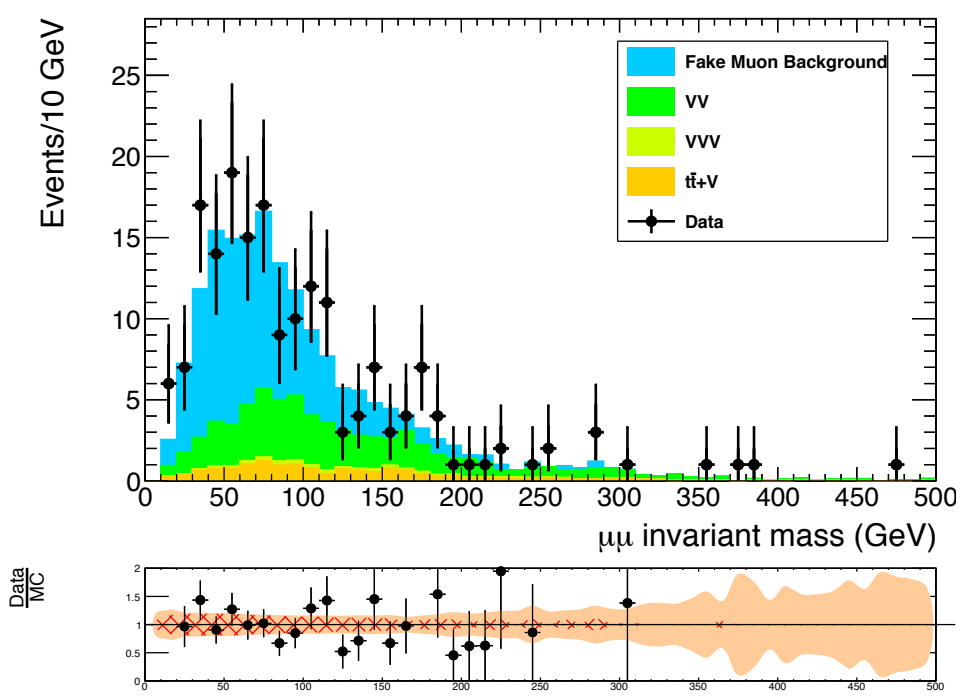

Figure 3.3: The invariant mass of the two selected muons at preselection. Light blue is the data-driven fake background, the others from MC simulation. The ratio plot includes the systematic uncertainty on the fake background (red hatches) and the total uncertainty (systematic and statistical combined) on the background estimate (orange shaded region). 


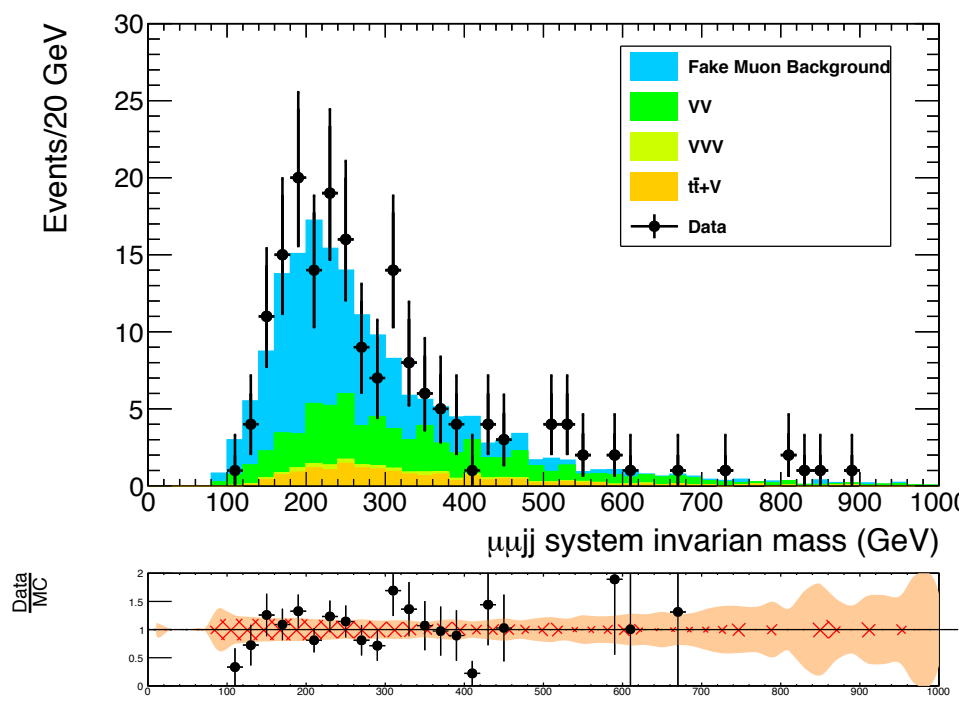

Figure 3.4: The invariant mass of the two selected muons and two selected jets at preselection. Light blue is the data-driven fake background, the others from MC simulation. The ratio plot includes the systematic uncertainty on the fake background (red hatches) and the total uncertainty (systematic and statistical combined) on the background estimate (orange shaded region). 


\subsubsection{Full Selection}

After pre-selection cuts are applied, the search is split into two mass regions. The first region, corresponding to a heavy Majorana neutrino with a mass between 40 and $90 \mathrm{GeV} / c^{2}$, is referred to as the:

\section{Low Mass Region}

and the second, corresponding to a mass $\geq 90 \mathrm{GeV} / c^{2}$, dubbed the:

\section{High Mass Region}

The motivation for this is to separate regions based on whether the W-boson propagator is on-shell or not.

In the low mass region, the $W$-propagator, (the first $\mathrm{W}$ boson in the Feynman diagram), is on-shell and the final state particles $\left(\mu^{ \pm} \mu^{ \pm} q \bar{q}^{\prime}\right)$ should have an invariant mass close to the mass of the $W$. The following is a list of the selection criteria for the low mass region:

(a) Low Mass Majorana Neutrino Selection $\left(40 \mathrm{GeV} / \mathrm{c}^{2}<M_{N}<90 \mathrm{GeV} / \mathrm{c}^{2}\right)$

- Any events with one or more b-tags (using the CSV Medium working point) are excluded (see Section 2.4.4).

- Missing transverse energy (MET) must be less than $30 \mathrm{GeV}$.

- The invariant mass of the two muons and the two jets $m(\mu \mu j j)<200$ $\mathrm{GeV} / c^{2}$, where the two jets chosen are those which result in $m\left(\mu^{ \pm} \mu^{ \pm} j j\right)$ closest to the $W$ mass.

- The invariant mass of two jets $m(j j)$ must be $<120 \mathrm{GeV} / c^{2}$, where the 
two jets used are the same ones selected for the $m\left(\mu^{ \pm} \mu^{ \pm} j j\right)$ cut.

In the high mass search region, $\left(m_{N} \geq 90 \mathrm{GeV} / c^{2}\right)$, the $\mathrm{W}$-propagator is offshell but the W-boson from the HMN decay is on-shell, meaning the $W \rightarrow q \bar{q}^{\prime}$ decay should result in a dijet invariant mass close to $m_{W}$. In this region the following selection cuts are required:

(b) High Mass Majorana Neutrino Selection $\left(M_{N} \geq 90 \mathrm{GeV} / c^{2}\right)$

- Any events with one or more b-tags (using the CSV Medium working point) are excluded (see Section 2.4.4).

- The dimuon invariant mass $m(\mu \mu)$ must be $>15 \mathrm{GeV} / c$ (in order to completely exclude the $\Upsilon$ ).

- The missing transverse energy (MET) must be $<35 \mathrm{GeV}$.

- The dijet invariant mass must satisfy $50<m(j j)<110 \mathrm{GeV} / c^{2}$, where the two jets with an invariant mass closest to the $W$ mass are chosen.

In order to understand the background, a control region was defined using the same event signature, while requiring an independent variable that ensures the signal is excluded. For example, there should be no b-quarks generated in this signal, and by requiring a b-tagged jet in the control region, any events studied should consist only of background processes to the signal of interest.

The following criteria define the control regions used:

(a) Low Mass Control Region $\left(40 \mathrm{GeV} / c^{2}<M_{N}<90 \mathrm{GeV} / c^{2}\right)$

- The selection is the same as the low mass signal selection, except: 
- (missing transverse energy (MET) must be $>50 \mathrm{GeV}$ ) OR (one or more b-tags must pass the CSV Medium working point, see Section 2.4.4)

(b) High Mass Control Region $\left(M_{N} \geq 90 \mathrm{GeV} / c^{2}\right)$

- The selection is the same as the high mass signal selection, except:

- (The missing transverse energy (MET) must be $>50 \mathrm{GeV}$ ) OR (one or more b-tags must pass the CSV Medium working point, see Section 2.4.4)

\subsubsection{Final Selection Optimization}

In the interest of completeness, the final selection optimization procedure is briefly described here, though the results are described in Section 3.7.

A figure of merit was used to optimize the final signal selection criteria as a function of HMN mass. The figure of merit quantifies the goodness of the selection by comparing the signal with background while also incorporating the statistical significance of the expected signal. The selection was optimized with the following three variables:

- The transverse momentum of the leading muon, $p_{T, \mu 1}$.

- The transverse momentum of the trailing muon, $p_{T, \mu 2}$.

- The invariant mass of the two muons and two selected jets $m(\mu \mu j j)$.

with the Punzi (see [59]) figure of merit:

$$
\frac{\epsilon_{S}}{a / 2+\sqrt{N_{t}+\left(\delta N_{f}\right)^{2}}}
$$

Here, $\epsilon_{S}$ is the signal efficiency, $a$ is the number of standard deviations desired from 
the result (in this case $a=2$ as is standard), and $\delta$ is the fractional systematic uncertainty on the fake background estimate (see Section 3.6.1). The second term in the denominator is the standard uncertainty on the background estimate plus - in this specific case - a fixed contribution of $28 \%$ due to fake events, $\left(N_{f}\right)$. The results of this process are discussed in Section 3.7.

\subsection{Selection Efficiency}

This section summarizes the efficiencies of the various selection criteria. Each selection criteria's efficiency depends on specific traits intrinsic to the selection mechanism. In other words, this analysis requires the selection of two muons, but what exactly is a muon according to CMS? After all, CMS simply responds to a muon or other any other particle traversing its volume with a bundle of electrical pulses. How is it that one bundle of electrical pulses can be determined to be an electron, while another bundle can be called a muon? Increasing selection variables lead to increased confidence that the choice of particle being represented by the bundle of pulses is correct, but it also decreases the muon selection efficiency, hurting the overall statistics. Not all muons create the same bundle of electrical pulses, and not all analyses require the same strictness. The qualities used in this analysis to select the final particles, along with their efficiencies, are described below.

\subsubsection{Trigger Efficiency}

The muon trigger selection efficiencies along with their associated systematic uncertainties are taken directly from the muon POG, as detailed in Reference [60]. 
The muon trigger efficiency is $\mathrm{p}_{T}$ and $\eta$ dependent, and is above $90 \%$ for both the low mass and high mass search regions.

\subsubsection{Offline Selection Efficiency}

One of the most important studies in this analysis was the estimate of the selection efficiencies for the event signature as a function of HMN mass. The goal is to understand the efficiencies of selecting two jets and two muons, and with all the described selection criteria.

First, described in Table 3.5, is the selection efficiency of the same-sign muons.

Of note is the dip in selection efficiency when the HMN mass is between 70 and 90 $\mathrm{GeV} / c^{2}$. This is due to the nature of the production process; if the HMN is near the $W$ mass, the produced muons will be too soft; i.e., their $\mathrm{p}_{T}$ will be too low to pass our selection criteria, leading to a drop in efficiency.

To calculate these efficiencies, a suite of software was written to create nTuples of the signal Monte Carlo samples filled with quantities the analysis would use for selection criteria. A separate analysis package was written to analyze the data and calculate the efficiencies for the final state particles with very fine resolution, allowing the understanding of which cuts were causing the largest drops in efficiencies. This process resulted in high optimization of the preselection criteria.

The overall selection efficiency of the signal signature is listed in Table 3.6. Also in Table 3.6 are the estimated numbers of events expected in our dataset with an integrated luminosity of $19.7 \mathrm{fb}^{-1}$. 
Table 3.5: Muon selection and individual cut efficiencies for events in Signal MC passing the selection cuts listed in Sections 3.4.3 - 3.4.6.

\begin{tabular}{lccccc}
\hline Generated & Total & & \multicolumn{3}{c}{ \% Events Accepted } \\
HMN Mass & Events & Track Quality & $\eta$ and $\mathrm{p}_{T}$ & Isolation & All \\
\hline $40 \mathrm{GeV} / c^{2}$ & 99390 & $46.0 \pm 0.2$ & $15.8 \pm 0.1$ & $21.5 \pm 0.1$ & $7.12 \pm 0.08$ \\
$60 \mathrm{GeV} / c^{2}$ & 99494 & $49.3 \pm 0.2$ & $11.0 \pm 0.1$ & $21.2 \pm 0.1$ & $4.72 \pm 0.07$ \\
$70 \mathrm{GeV} / c^{2}$ & 49994 & $44.9 \pm 0.2$ & $3.25 \pm 0.08$ & $17.0 \pm 0.2$ & $1.37 \pm 0.05$ \\
$80 \mathrm{GeV} / c^{2}$ & 98492 & $37.1 \pm 0.2$ & $12.1 \pm 0.1$ & $15.7 \pm 0.1$ & $5.50 \pm 0.07$ \\
$90 \mathrm{GeV} / c^{2}$ & 49996 & $50.2 \pm 0.2$ & $10.0 \pm 0.1$ & $19.0 \pm 0.2$ & $3.62 \pm 0.08$ \\
$100 \mathrm{GeV} / c^{2}$ & 49996 & $59.7 \pm 0.2$ & $29.3 \pm 0.2$ & $27.5 \pm 0.2$ & $12.3 \pm 0.2$ \\
$125 \mathrm{GeV} / c^{2}$ & 49995 & $67.4 \pm 0.2$ & $56.6 \pm 0.2$ & $40.5 \pm 0.2$ & $30.2 \pm 0.2$ \\
$150 \mathrm{GeV} / c^{2}$ & 49995 & $71.5 \pm 0.2$ & $65.2 \pm 0.2$ & $47.5 \pm 0.2$ & $37.7 \pm 0.2$ \\
$175 \mathrm{GeV} / c^{2}$ & 49995 & $74.9 \pm 0.2$ & $69.9 \pm 0.2$ & $52.3 \pm 0.2$ & $42.2 \pm 0.2$ \\
$200 \mathrm{GeV} / c^{2}$ & 49992 & $77.3 \pm 0.2$ & $73.7 \pm 0.2$ & $55.7 \pm 0.2$ & $45.7 \pm 0.2$ \\
$250 \mathrm{GeV} / c^{2}$ & 49997 & $81.0 \pm 0.2$ & $78.0 \pm 0.2$ & $58.7 \pm 0.2$ & $48.7 \pm 0.2$ \\
$300 \mathrm{GeV} / c^{2}$ & 49996 & $83.4 \pm 0.2$ & $81.0 \pm 0.2$ & $60.7 \pm 0.2$ & $50.4 \pm 0.2$ \\
$350 \mathrm{GeV} / c^{2}$ & 49995 & $84.9 \pm 0.2$ & $82.5 \pm 0.2$ & $60.7 \pm 0.2$ & $50.1 \pm 0.2$ \\
$400 \mathrm{GeV} / c^{2}$ & 49996 & $85.8 \pm 0.2$ & $83.8 \pm 0.2$ & $60.7 \pm 0.2$ & $50.3 \pm 0.2$ \\
$500 \mathrm{GeV} / c^{2}$ & 49995 & $86.4 \pm 0.2$ & $84.2 \pm 0.2$ & $59.1 \pm 0.2$ & $48.6 \pm 0.2$ \\
\hline
\end{tabular}

Note: Errors are statistical only. 
Table 3.6: Event selection efficiencies for the events in Signal MC passing the selection cuts listed in Sections 3.4.3 - 3.4.6.

\begin{tabular}{lccccl}
\hline Generated & Total & \multicolumn{3}{c}{ \% Events Accepted } & \# of Events \\
HMN Mass & Events & All Muon & Jets & All Cuts & for $19.7 \mathrm{fb}^{-1}$ \\
\hline $40 \mathrm{GeV} / c^{2}$ & 99390 & $7.12 \pm 0.08$ & $19.3 \pm 0.1$ & $0.741 \pm 0.027$ & $2216670 \pm 8290$ \\
$60 \mathrm{GeV} / c^{2}$ & 99494 & $4.72 \pm 0.07$ & $23.3 \pm 0.1$ & $0.722 \pm 0.027$ & $86654 \pm 3281$ \\
$70 \mathrm{GeV} / c^{2}$ & 49994 & $1.37 \pm 0.05$ & $30.1 \pm 0.2$ & $0.278 \pm 0.024$ & $11646 \pm 1004$ \\
$80 \mathrm{GeV} / c^{2}$ & 98942 & $5.50 \pm 0.07$ & $42.5 \pm 0.2$ & $1.233 \pm 0.035$ & $4644 \pm 135$ \\
$90 \mathrm{GeV} / c^{2}$ & 49996 & $3.62 \pm 0.08$ & $55.8 \pm 0.2$ & $0.788 \pm 0.04$ & $1106 \pm 56$ \\
$100 \mathrm{GeV} / c^{2}$ & 49996 & $12.3 \pm 0.2$ & $58.5 \pm 0.2$ & $4.970 \pm 0.097$ & $3500 \pm 69$ \\
$125 \mathrm{GeV} / c^{2}$ & 49995 & $30.2 \pm 0.2$ & $65.2 \pm 0.2$ & $13.15 \pm 0.151$ & $2798 \pm 33$ \\
$150 \mathrm{GeV} / c^{2}$ & 49995 & $37.7 \pm 0.2$ & $70.1 \pm 0.2$ & $16.72 \pm 0.167$ & $1518 \pm 15$ \\
$175 \mathrm{GeV} / c^{2}$ & 49995 & $42.2 \pm 0.2$ & $73.6 \pm 0.2$ & $19.14 \pm 0.176$ & $880 \pm 8$ \\
$200 \mathrm{GeV} / c^{2}$ & 49992 & $45.7 \pm 0.2$ & $76.6 \pm 0.2$ & $20.88 \pm 0.182$ & $542 \pm 5$ \\
$250 \mathrm{GeV} / c^{2}$ & 49997 & $48.7 \pm 0.2$ & $80.4 \pm 0.2$ & $22.84 \pm 0.188$ & $230 \pm 3$ \\
$300 \mathrm{GeV} / c^{2}$ & 49996 & $50.4 \pm 0.2$ & $82.5 \pm 0.2$ & $23.39 \pm 0.189$ & $107.3 \pm 0.9$ \\
$350 \mathrm{GeV} / c^{2}$ & 49995 & $50.1 \pm 0.2$ & $83.5 \pm 0.2$ & $22.71 \pm 0.187$ & $52.5 \pm 0.5$ \\
$400 \mathrm{GeV} / c^{2}$ & 49996 & $50.3 \pm 0.2$ & $83.6 \pm 0.2$ & $22.31 \pm 0.186$ & $27.9 \pm 0.3$ \\
$500 \mathrm{GeV} / c^{2}$ & 49995 & $48.6 \pm 0.2$ & $82.4 \pm 0.2$ & $19.70 \pm 0.178$ & $8.4 \pm 0.1$ \\
\hline
\end{tabular}

Note: Errors are statistical only. 


\subsubsection{Tag and Probe Study}

One of the most intensive aspects of this analysis was a so-called tag and probe study. The tag and probe method for this analysis is used to compare muon selection efficiencies in data and MC in order to calculate the so-called Data/MC scale factor. ${ }^{7}$

The tag and probe method is a data-driven technique used to calculate efficiencies. In this analysis, the efficiency of the muon reconstruction is an important quantity, and with our signal sample created using a Monte Carlo generator, the MC performance must be compared with data to ensure the accuracy of the simulation. This directly effects the estimation of the signal. Normally, the muon POG takes care of these calculations and the user can simply incorporate the efficiencies and scale factors directly into their analysis. However, during the early phases of the analysis, it was discovered that a lower Relative Isolation (RelIso) than the standard POG value improved the figure of merit. Therefore, the tag and probe method was deployed to understand the effect of tightening this requirement on muon efficiency in both data and MC.

The method requires a known mass resonance with preferably a low background. The subject of the analysis is the muon, making a perfect candidate of the $Z \rightarrow \mu^{ \pm} \mu^{\mp}$ Drell-Yan process. The tag and the probe are a tight and a loose muon, respectively.

The method begins by choosing a muon with very tight selection requirements as the tag, and a probe muon with only basic qualities like minimum $\mathrm{p}_{T}$ and $\eta$

\footnotetext{
${ }^{7}$ For an in-depth description of the tag and probe method, see [61, 62, 63, 64].
} 
requirements. The efficiency is defined as:

$$
\epsilon=\frac{T P}{(T P+T F)}
$$

where TP represents the two muons: The first letter in a pair representing the tag muon, and the second letter representing the state defining whether or not the probe muon passed or failed. $\mathrm{TP}=$ Tag and passing probe, $\mathrm{TF}=$ Tag and failing probe.

Only events containing exactly 2 muons were considered and only events with muons having opposite charge were considered ${ }^{8}$. After an event is found containing two "loose" muons, the algorithm checks to see if one of them matches the muon which triggered the event (with $\Delta \mathrm{R}$ matching and a truth boolean in the ntuple (labeled MuonHLTSingleIsoMuonMatched)), and then asks if the triggered muon passes the tight tag requirements. If it does, its properties and ID are stored and its index is labeled as the tag muon.

Next it checks to see if the second muon, which has already passed the 'probe' criteria, also passes the 'tight' criteria. If it passes, the tag/probe pair's combined mass is stored in a histogram labeled 'Pass'. If it fails, the tag/probe pair's mass is stored into a histogram labeled 'Fail'.

After populating the pass and fail histograms by running over all the events, the efficiency can be calculated by determining the number of events which pass and the number of events which fail. In order to differentiate true $\mathrm{Z}$ decay muons from background muons, the pass/fail yields are determined by using fits that are applied to the histograms. The histograms are fit using the RooFit package with a sum of two

\footnotetext{
${ }^{8}$ This was required in order to reduce selecting muons from other processes.
} 
voigtian functions representing the signal, and an exponential function representing the background ${ }^{9}$. The mean of the fits are bound to $90-92 \mathrm{GeV} / \mathrm{c}^{2}$.

Figures 3.5 and 3.6 show examples of the $\mathrm{Z}$ mass peak fits used to estimate the signal yield. In these figures, the left plot is used to determine the TP quantity, and the right plot is used to determine the TF quantity.

In order to understand the efficiency as a function of muon $\mathrm{p}_{T}$, this process was repeated for 12 separate $\mathrm{p}_{T}$ regions, and 4 distinct $\eta$ regions. The final efficiencies for both data and $\mathrm{MC}$, including their scale factors (data/MC), as a function of $\mathrm{p}_{T}$ and $\eta$, can be studied in Figures 3.7 and 3.8. This same information is displayed in tabular form in Tables 3.7 and 3.8.

These results (with scale factors close to 1) show that the muons in our selected range are well modeled by Monte Carlo simulations. The scale factors are then used to rescale the $\mathrm{MC}$ datasets ${ }^{10}$.

\subsection{Background Estimation}

The main backgrounds in this analysis concern events in which either one muon is fake (e.g. $t \bar{t}$ and $W+$ jets) or both muons are fake (e.g. QCD multijet events). In the former case, one muon originates from a $W$ decay and is a real prompt muon, while the second muon comes from a jet (likely a $b$ jet, though these muons are typically less isolated). In the latter case, both muons come from jets and are both non-prompt

\footnotetext{
${ }^{9}$ This is to accurately account for both the $\mathrm{Z}$ decay shape and the distribution widening from detector resolution effects.

${ }^{10}$ An overall systematic of $2 \%$ was used for the muon selection, as described in Section 3.8 .
} 

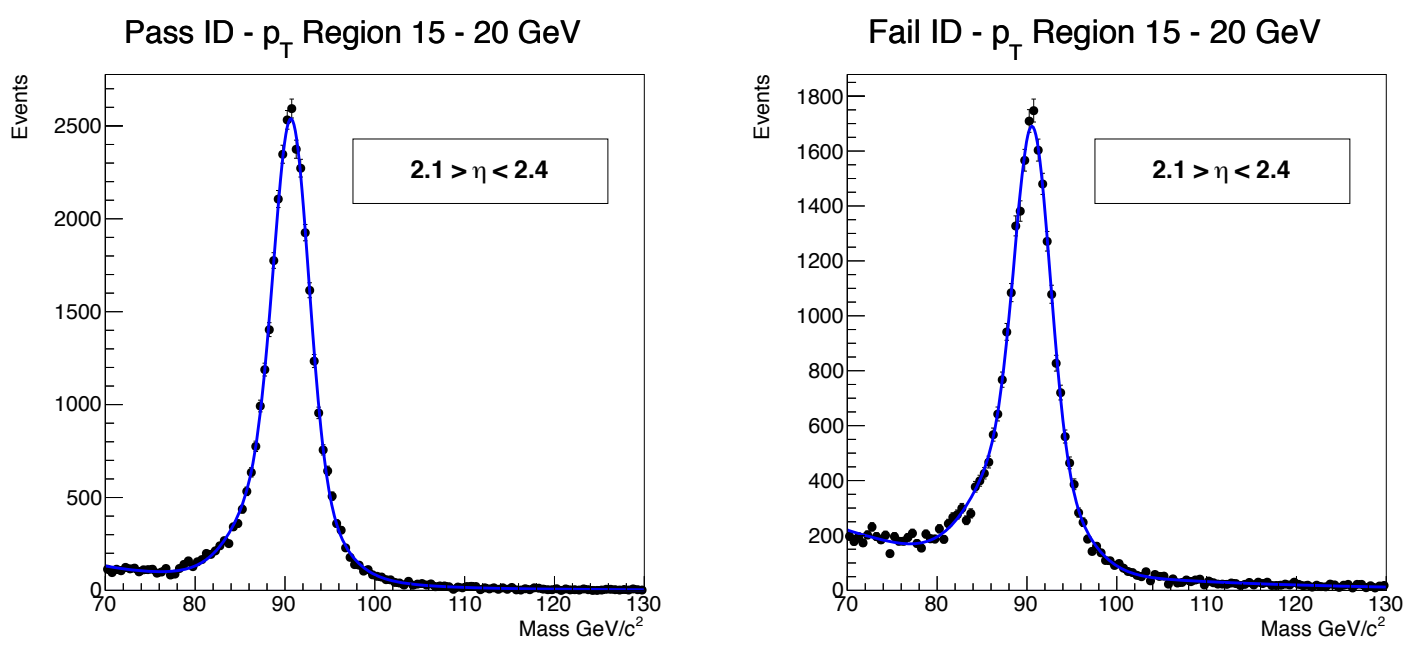

Figure 3.5: Example $\mathrm{Z}$ Boson mass peak from $Z \rightarrow \mu \mu$ in collision data. Pass (left) and fail (right) histograms used in the tag and probe study. See Section 3.5.3 for a detailed description.
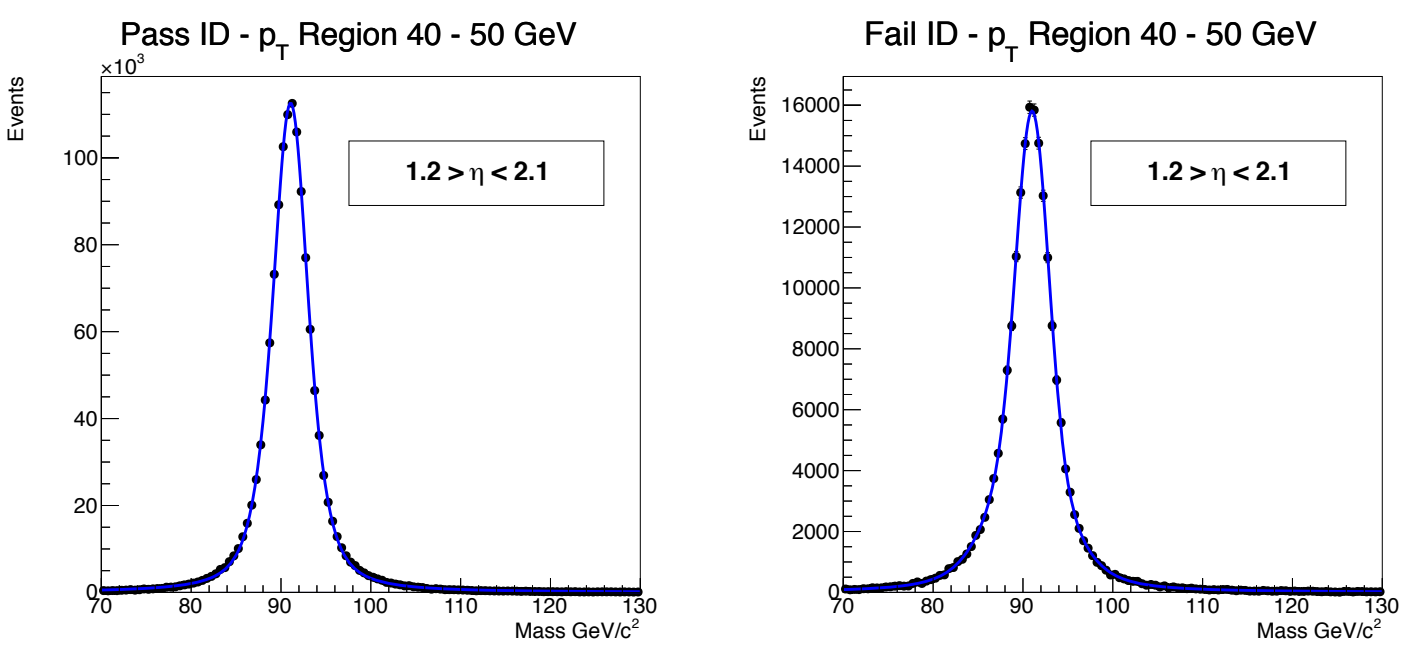

Figure 3.6: Example Z Boson mass peak from $Z \rightarrow \mu \mu$ in Monte Carlo data. Pass (left) and fail (right) histograms used in the tag and probe study. See Section 3.5.3 for a detailed description. 
Tight Muon ID $-\mathrm{m} \eta \mathrm{l}<0.9$
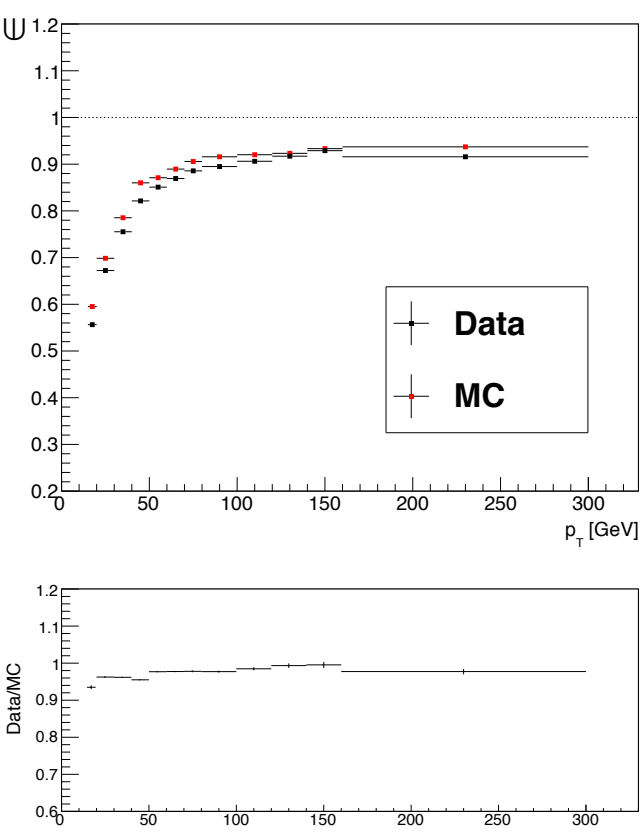

Tight Muon ID - $0.9<|\eta|<1.2$
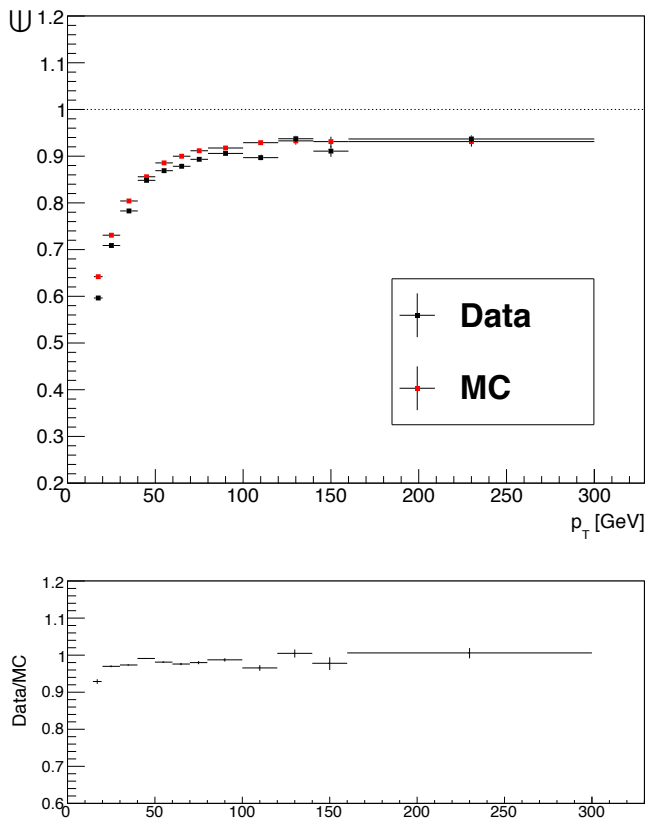

Figure 3.7: Muon Efficiencies using the Tag and Probe method for $|\eta| 0-1.2$.

Tight Muon ID $-1.2<|\eta|<2.1$
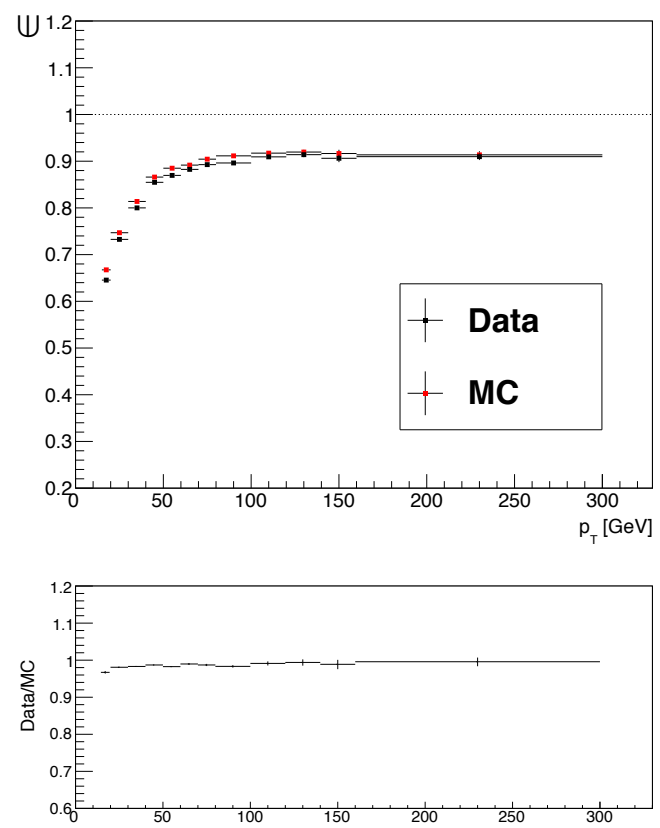

Tight Muon ID $-2.1<h \mid<2.4$
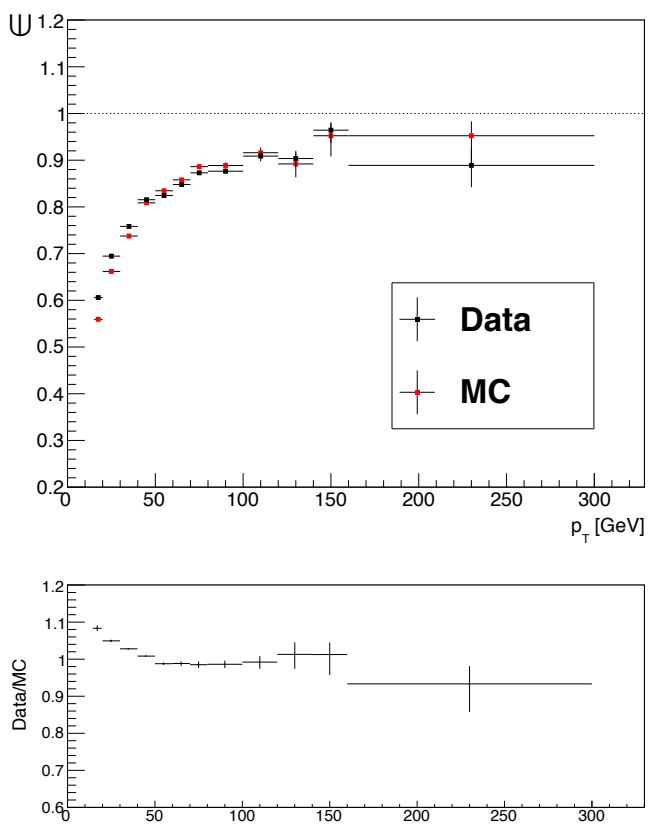

Figure 3.8: Muon Efficiencies using the Tag and Probe method for $|\eta| 1.2-2.4$. 
Table 3.7: Tag and Probe Muon ID efficiencies for MC and data, $\eta$ region 1 and 2.

\begin{tabular}{ccccc}
\hline$|\eta|$ & $\mathrm{p}_{T}$ range $(\mathrm{GeV} / \mathrm{c})$ & Data & $\mathrm{MC}$ & Data/MC \\
\hline$<0.9$ & $15-20$ & $0.556 \pm 0.002$ & $0.595 \pm 0.003$ & $0.935 \pm 0.005$ \\
$20-30$ & $0.672 \pm 0.001$ & $0.698 \pm 0.001$ & $0.962 \pm 0.001$ \\
$30-40$ & $0.755 \pm 0.000$ & $0.785 \pm 0.000$ & $0.962 \pm 0.001$ \\
$40-50$ & $0.821 \pm 0.000$ & $0.860 \pm 0.000$ & $0.955 \pm 0.000$ \\
$50-60$ & $0.851 \pm 0.001$ & $0.871 \pm 0.001$ & $0.977 \pm 0.001$ \\
$60-70$ & $0.869 \pm 0.001$ & $0.889 \pm 0.001$ & $0.977 \pm 0.002$ \\
$70-80$ & $0.886 \pm 0.001$ & $0.906 \pm 0.002$ & $0.978 \pm 0.002$ \\
$80-100$ & $0.895 \pm 0.002$ & $0.916 \pm 0.002$ & $0.977 \pm 0.003$ \\
$100-120$ & $0.906 \pm 0.003$ & $0.920 \pm 0.003$ & $0.985 \pm 0.004$ \\
$120-140$ & $0.917 \pm 0.004$ & $0.923 \pm 0.004$ & $0.993 \pm 0.006$ \\
$140-160$ & $0.929 \pm 0.005$ & $0.933 \pm 0.006$ & $0.995 \pm 0.008$ \\
$160-300$ & $0.916 \pm 0.005$ & $0.937 \pm 0.005$ & $0.977 \pm 0.007$ \\
\hline $15-20$ & $0.596 \pm 0.003$ & $0.642 \pm 0.003$ & $0.929 \pm 0.006$ \\
& $20-30$ & $0.709 \pm 0.001$ & $0.731 \pm 0.001$ & $0.970 \pm 0.002$ \\
$30-40$ & $0.783 \pm 0.001$ & $0.804 \pm 0.001$ & $0.973 \pm 0.001$ \\
$40-50$ & $0.848 \pm 0.000$ & $0.856 \pm 0.001$ & $0.991 \pm 0.001$ \\
$50-60$ & $0.869 \pm 0.001$ & $0.886 \pm 0.001$ & $0.981 \pm 0.002$ \\
$60-70$ & $0.878 \pm 0.002$ & $0.900 \pm 0.002$ & $0.976 \pm 0.003$ \\
& $70-80$ & $0.893 \pm 0.002$ & $0.912 \pm 0.003$ & $0.980 \pm 0.004$ \\
$80-100$ & $0.906 \pm 0.003$ & $0.918 \pm 0.003$ & $0.987 \pm 0.005$ \\
$100-120$ & $0.897 \pm 0.005$ & $0.929 \pm 0.005$ & $0.966 \pm 0.008$ \\
$120-140$ & $0.937 \pm 0.007$ & $0.933 \pm 0.008$ & $1.005 \pm 0.011$ \\
$140-160$ & $0.911 \pm 0.012$ & $0.931 \pm 0.011$ & $0.978 \pm 0.017$ \\
$160-300$ & $0.937 \pm 0.008$ & $0.931 \pm 0.010$ & $1.006 \pm 0.014$ \\
\hline & & &
\end{tabular}

Note: Errors are statistical only. 
Table 3.8: Tag and Probe Muon ID efficiencies for MC and data, $\eta$ region 3 and 4.

\begin{tabular}{ccccc}
\hline$|\eta|$ & $\mathrm{p}_{T}$ range $(\mathrm{GeV} / \mathrm{c})$ & Data & $\mathrm{MC}$ & Data/MC \\
\hline $1.2-2.1$ & $15-20$ & $0.645 \pm 0.002$ & $0.667 \pm 0.002$ & $0.967 \pm 0.003$ \\
$20-30$ & $0.733 \pm 0.001$ & $0.747 \pm 0.001$ & $0.981 \pm 0.001$ \\
$30-40$ & $0.800 \pm 0.000$ & $0.814 \pm 0.001$ & $0.983 \pm 0.001$ \\
$40-50$ & $0.855 \pm 0.000$ & $0.866 \pm 0.000$ & $0.987 \pm 0.001$ \\
& $50-60$ & $0.870 \pm 0.001$ & $0.885 \pm 0.001$ & $0.983 \pm 0.001$ \\
$60-70$ & $0.882 \pm 0.001$ & $0.892 \pm 0.001$ & $0.990 \pm 0.002$ \\
& $70-80$ & $0.893 \pm 0.002$ & $0.904 \pm 0.002$ & $0.987 \pm 0.003$ \\
$80-100$ & $0.896 \pm 0.002$ & $0.911 \pm 0.002$ & $0.983 \pm 0.003$ \\
$100-120$ & $0.909 \pm 0.004$ & $0.917 \pm 0.004$ & $0.991 \pm 0.005$ \\
$120-140$ & $0.914 \pm 0.005$ & $0.920 \pm 0.006$ & $0.994 \pm 0.008$ \\
$140-160$ & $0.906 \pm 0.008$ & $0.916 \pm 0.008$ & $0.989 \pm 0.013$ \\
$160-300$ & $0.910 \pm 0.007$ & $0.914 \pm 0.008$ & $0.996 \pm 0.012$ \\
\hline $2.1-2.4$ & $15-20$ & $0.606 \pm 0.003$ & $0.559 \pm 0.003$ & $1.083 \pm 0.008$ \\
& $20-30$ & $0.695 \pm 0.001$ & $0.662 \pm 0.001$ & $1.049 \pm 0.003$ \\
& $30-40$ & $0.758 \pm 0.001$ & $0.738 \pm 0.001$ & $1.028 \pm 0.002$ \\
& $40-50$ & $0.815 \pm 0.001$ & $0.809 \pm 0.001$ & $1.008 \pm 0.002$ \\
$50-60$ & $0.824 \pm 0.002$ & $0.835 \pm 0.002$ & $0.988 \pm 0.003$ \\
$60-70$ & $0.848 \pm 0.003$ & $0.858 \pm 0.004$ & $0.988 \pm 0.006$ \\
& $70-80$ & $0.873 \pm 0.005$ & $0.886 \pm 0.006$ & $0.985 \pm 0.009$ \\
$80-100$ & $0.876 \pm 0.006$ & $0.889 \pm 0.007$ & $0.986 \pm 0.010$ \\
$100-120$ & $0.909 \pm 0.010$ & $0.916 \pm 0.012$ & $0.992 \pm 0.017$ \\
$120-140$ & $0.904 \pm 0.018$ & $0.892 \pm 0.026$ & $1.013 \pm 0.036$ \\
$140-160$ & $0.964 \pm 0.022$ & $0.952 \pm 0.035$ & $1.013 \pm 0.044$ \\
$160-300$ & $0.889 \pm 0.041$ & $0.952 \pm 0.045$ & $0.933 \pm 0.062$ \\
\hline
\end{tabular}

Note: Errors are statistical only. 
fake muons.

To handle the background estimation, a data driven method is used, referred to as the fake rate method, described shortly in Section 3.6.1.

Monte Carlo simulations generally are not a reliable tool to estimate the fake background, due to the inexact modeling of the parton showering process along with the low probability of getting a jet to 'fake' a muon in $\mathrm{MC}$.

As an initial check, Monte Carlo samples are analyzed using the event signature of two same-sign muons and two jets ${ }^{11}$ (Section 3.4.5) in order to estimate the fake rate, within an order of magnitude.

Using the selections described in Section 3.4.5, the fake background is found to be comprised of $60 \%$ QCD multijet production, $30 \% W+$ jets, and $15 \% t \bar{t}$. (The last two backgrounds are a much smaller fraction of the total fake background for the final signal selection because of the cuts on MET and the $b$-tag veto. $)^{12,13}$

\subsubsection{The Fake Rate Method}

The fake rate method is used to estimate the background due to fake muons. The fake rate method is detailed in Reference [65] and has been used in previous

\footnotetext{
${ }^{11}$ Only the preselection criteria are used in order to retain as many events for statistical value.

${ }^{12}$ Sources of real same-sign dimuon events in the Standard Model are also considered. This 'irreducible' background consists of diboson events $(W Z, Z Z)$, same-sign $W W$, triboson $W W W$ or $Z Z Z$, and $t \bar{t}$ plus boson $(t \bar{t} W, t \bar{t} Z, t \bar{t} W W)$. These processes have very small cross sections, making their contributions well below that of the background from fakes. They are also estimated using Monte Carlo samples.

${ }^{13}$ The background from muon charge mis-measurement is also considered and found to be negligible.
} 
analyses; see for example Reference [66]. A very basic description of the method will now be reviewed.

An independent data sample dominated by QCD jets representing the measurement region is used to calculate a fake rate (referred to as the tight-to-loose ratio or $\mathrm{T} / \mathrm{L}$ ratio). The fake rate is defined as the fraction of muon candidates passing loose cuts that also pass the tight cuts used in the analysis to select the final event sample:

$$
F R\left(p_{T}, \eta\right)=\frac{\# \text { of muons passing loose and tight selection criteria }}{\# \text { of muons passing loose selection criteria }}
$$

Muon candidates that pass the loose cuts are referred to as "fakeable objects". The fake rate is calculated as a function of the fakeable object's transverse momentum $\left(\mathrm{p}_{T}\right)$ and pseudorapidity $(\eta)$. The fake rate is then used as an event weight to calculate the background using a sample of events which pass all the signal selection cuts, except that one or both muons fail the tight selection. This sample is referred to as the "orthogonal" sample.

An estimate of the fake rate prediction is obtained by weighting each event in which one muon passes the tight cuts and the other muon fails the tight selection but passes the loose selection $\left(N_{n \bar{n}}\right)$ and the number of events in which both muons fail the tight selection but pass the loose cuts $\left(N_{\bar{n} \bar{n}}\right)$. The total contribution to the signal sample (in other words the number of events in which both muons pass the tight selection, $\left(N_{n n}\right)$, is obtained by summing all the weighted events as in Equation 3.8.

A simplified version of the method is implemented, assuming that the prob- 
ability for a real prompt muon to pass the tight selection is 1 . This assumption simplifies the method and reduces possible sources of systematic errors ${ }^{14}$.

The equations that relate the number of events with prompt-prompt $\left(N_{p p}\right)$, prompt-fake $\left(N_{p f}\right)$, and fake-fake $\left(N_{f f}\right)$ muons to $N_{n \bar{n}}, N_{\bar{n} \bar{n}}$, and $N_{n n}$ are:

$$
\begin{gathered}
N_{t o t}=N_{p p}+N_{p f}+N_{f f}=N_{n n}+N_{n \bar{n}}+N_{\bar{n} \bar{n}} \\
N_{\bar{n} \bar{n}}^{i j}=\left(1-F R_{i}\right)\left(1-F R_{j}\right) N_{f f}^{i j} \\
N_{n \bar{n}}^{i j}=(1-F R j) N_{p f}^{i j}+\left(F R_{i}\left(1-F R_{j}\right)+F R_{j}\left(1-F R_{i}\right)\right) N_{f f}^{i j} \\
N_{n n}^{i j}=N_{p p}^{i j}+F R_{j} N_{p f}^{i j}+F R_{i} F R_{j} N_{f f}^{i j}
\end{gathered}
$$

After some quick algebra, the fake contribution to the signal sample $N_{n n}$ can be derived to:

$$
\begin{aligned}
N_{n n}=\sum_{i, j} \frac{F R_{i} F R_{j}}{\left(1-F R_{i}\right)\left(1-F R_{j}\right)} N_{\bar{n} \bar{n}}^{i j} & \\
& \quad+\sum_{i, j} \frac{F R_{i}}{\left(1-F R_{i}\right)}\left[N_{n \bar{n}}^{i j}-\frac{F R_{i}\left(1-F R_{j}\right)+F R_{j}\left(1-F R_{i}\right)}{\left(1-F R_{i}\right)\left(1-F R_{j}\right)} N_{\bar{n} \bar{n}}^{i j}\right]
\end{aligned}
$$

where $F R$ is the fake rate, and $i, j$ label the two muons in the event. Note that in the square parentheses we have explicitly written the correction to $N_{n \bar{n}}^{i j}$ due to $\bar{n} \bar{n}$ events that can be also be $n \bar{n}$. In the hypothesis of no real sources of events with two prompt leptons, this term has to be identically zero as all the single fake events must be generated from double fake events (double-to-single).

\footnotetext{
${ }^{14}$ Even if a very tight ID is used, the contamination coming from same-sign prompt events (like $W Z$ ) in the orthogonal sample is on the order of $1 \%$ of the total number of events with one tight and one loose muon. As such it is not necessary to apply a correction for a contamination this small.
} 


\subsubsection{Fake Rate Measurement in Data}

Fakeable objects are defined by relaxing the muon ID requirements. The following loose cuts are used to select fakeable muons:

- $\chi^{2} /$ number of degrees of freedom (analysis cut $<10$, loose selection $<50$ );

- Transverse impact parameter $d_{x y}$ (analysis cut $<0.05 \mathrm{~mm}$, loose selection $<2$ $\mathrm{mm})$

- RelIso (analysis cut $<0.05$, loose selection $<0.4$ ).

Fakeable objects are defined as muon candidates passing the selection defined in 3.4.3, but with these three cuts relaxed.

The independent control sample used to obtain the fake rate is selected using the following single muon triggers:

- HLT_Mu8

- HLT_Mu12

- HLT_Mu17

- HLT_Mu24

The first two triggers are used for the $\mathrm{p}_{T}$ bin $10<\mathrm{p}_{T} \leq 20 \mathrm{GeV} / c$, the third for $20<\mathrm{p}_{T} \leq 25 \mathrm{GeV} / c$, and the last for $\mathrm{p}_{T}$ bins above $25 \mathrm{GeV} / c$.

The goal is to measure the fake rate in QCD events. By definition there are no tight muons in QCD events. Therefore, dijet-like events are selected. The selected events must contain one muon that passes the loose requirements (probe) and one jet $(\operatorname{tag})$ on the opposite side to the muon $\left(\Delta \phi_{\mu j}>2.5\right.$, as dijets are generally 
jets $p_{t}(\mathrm{GeV})$

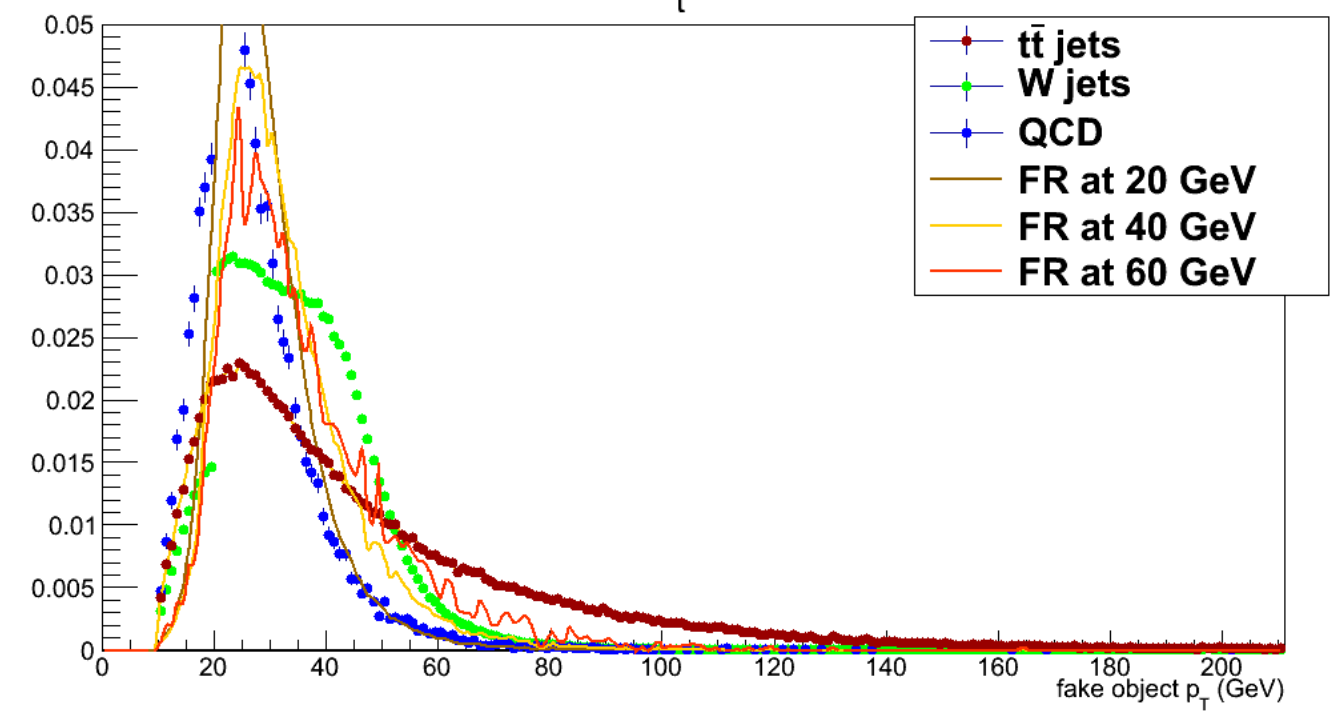

Figure 3.9: The $\mathrm{p}_{T}$ spectrum of jets tagged as a fake object in MC. Comparison between $t \bar{t}, W+$ jets, and QCD Monte Carlo. The shapes are normalized to unity.

distributed) that satisfies the same selection cuts used in the analysis except for the $\mathrm{p}_{T}$ requirement, which in this case is set at $\mathrm{p}_{T}>40 \mathrm{GeV} / c$. (This $\mathrm{p}_{T}$ cut is chosen to match the expected $\mathrm{p}_{T}$ distribution of the fake objects in the orthogonal sample as shown in Figure 3.9, which reflects the distribution expected in the signal region. More on Figure 3.9 in Section 3.6.3.)

In order to reject $W$ events, the cuts MET $<20 \mathrm{GeV}$ and transverse mass $M_{T}<25 \mathrm{GeV} / c^{2}$ are imposed. To suppress $Z$ events, a veto is applied to events with more than one muon. Dimuon events are checked using the full $M_{T}$ spectrum to check the normalization of the Drell-Yan and $W+$ jets $\mathrm{MC}$, after being normalized to the trigger luminosities of the four triggers, in what can be defined as a fake rate control 

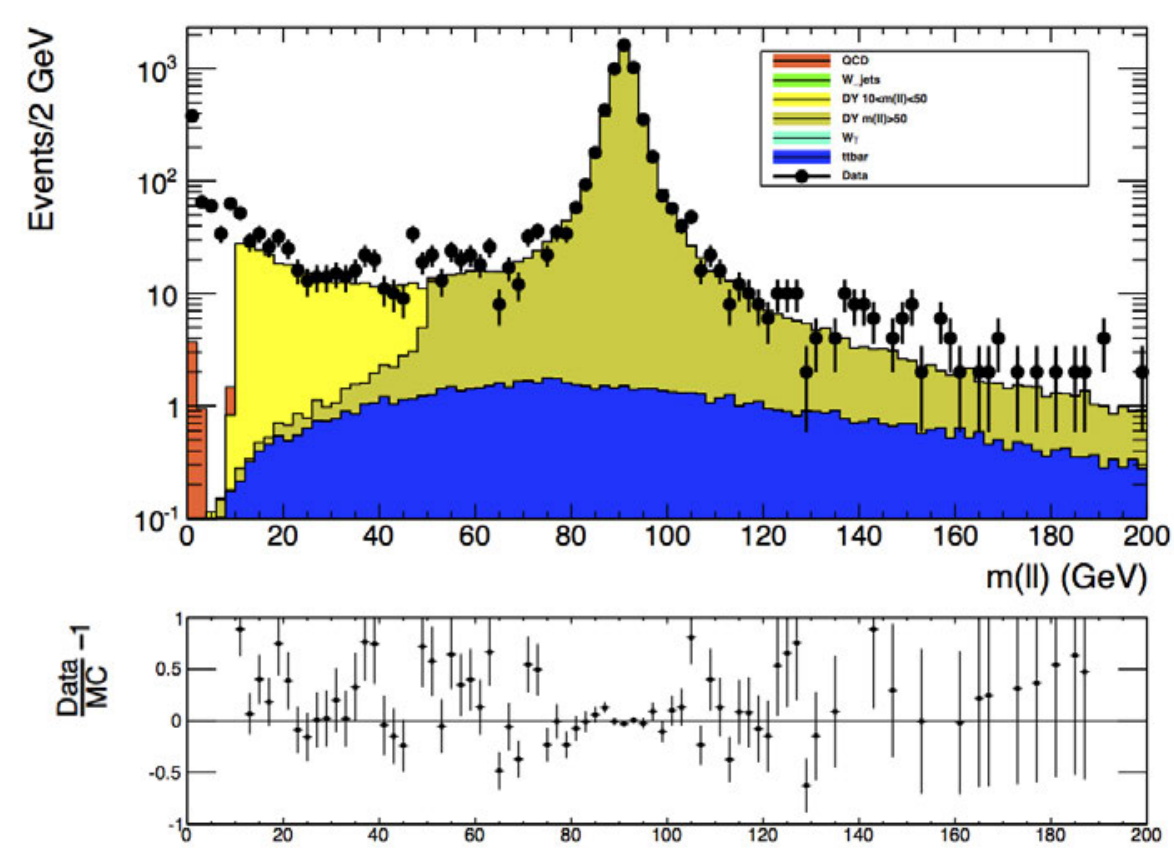

Figure 3.10: The $m(l l)$ distribution of the events in the fake-rate control region. The invariant mass, $m(l l)$, is calculated using the two leading leptons and requiring one jet above $40 \mathrm{GeV} / \mathrm{c}$.

region. Events with an invariant mass $m_{\mu \mu}$ within $20 \mathrm{GeV} / c^{2}$ of the $Z$ boson mass are only used to check the normalization of the Drell-Yan Monte Carlo (see Figure 3.10). The normalization of the $W+$ jets Monte Carlo is checked in the large $M_{T}$ region (Figure 3.11).

The fake rate is corrected for any remaining prompt contamination by subtracting the Monte Carlo contributions of $W, Z, t \bar{t}$ and $W \gamma$ processes from both the numerator (tight) and denominator (loose). The distributions of $\mathrm{p}_{T}$ and $\eta$ for the events in the numerator and denominator selections are plotted in Figures 3.12 and 

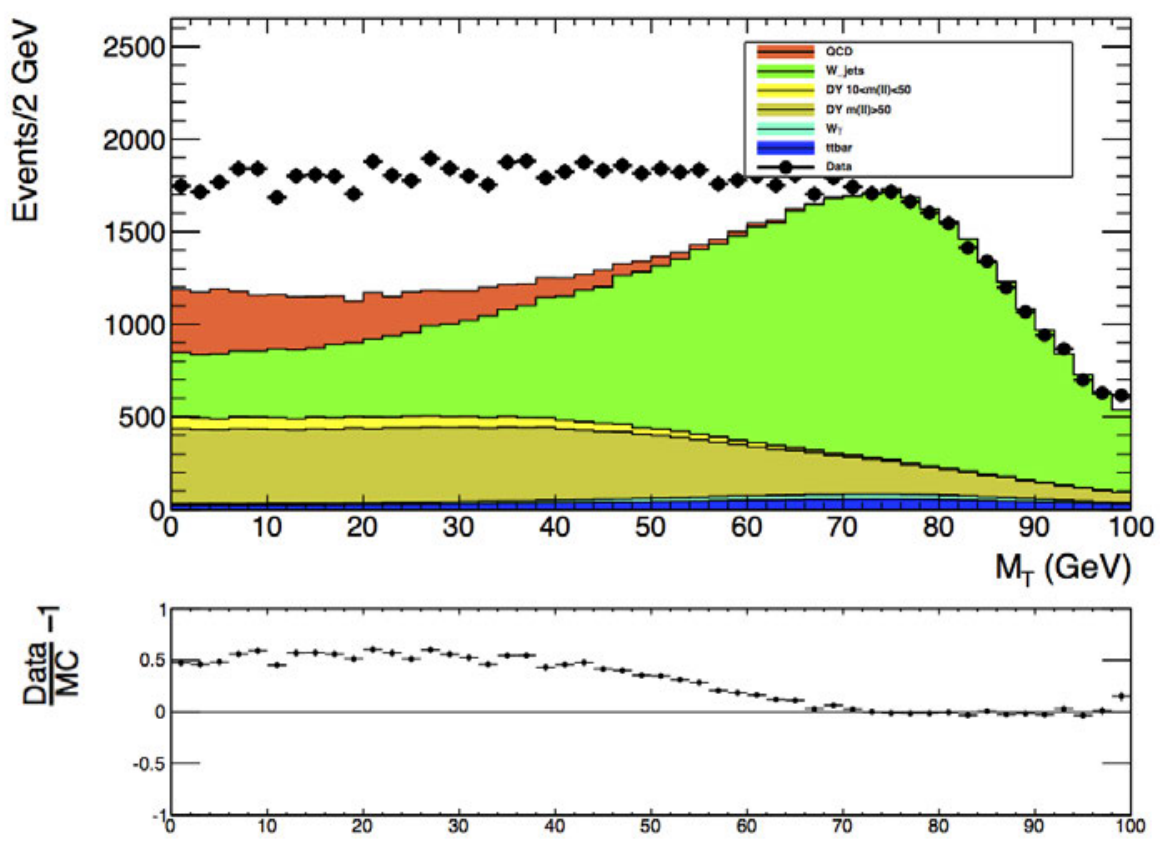

Figure 3.11: The transverse mass $\left(M_{T}\right)$ distribution of the events in the fake-rate control region. $M_{T}$ is calculated using the leading lepton and requiring one jet above $40 \mathrm{GeV} / \mathrm{c}$. The lack of events at small $M_{T}$ is due to lack of Monte Carlo events in the QCD sample. 
Table 3.9: Muon fake rates obtained from data in bins of $|\eta|$ and $\mathrm{p}_{T}$.

\begin{tabular}{c|cccc}
\hline $\mathrm{p}_{T}$ Range & \multicolumn{4}{|c}{$|\eta|$ Region } \\
$(\mathrm{GeV} / \mathrm{c})$ & $0-1.0$ & $1.0-1.479$ & $1.479-2.0$ & $2.0-2.5$ \\
\hline $15-20$ & $0.052 \pm 0.007$ & $0.060 \pm 0.008$ & $0.080 \pm 0.011$ & $0.089 \pm 0.005$ \\
$20-25$ & $0.050 \pm 0.004$ & $0.050 \pm 0.005$ & $0.079 \pm 0.007$ & $0.070 \pm 0.012$ \\
$25-30$ & $0.044 \pm 0.004$ & $0.051 \pm 0.004$ & $0.071 \pm 0.006$ & $0.091 \pm 0.013$ \\
$30-35$ & $0.044 \pm 0.004$ & $0.066 \pm 0.008$ & $0.068 \pm 0.010$ & $0.080 \pm 0.019$ \\
$35-45$ & $0.067 \pm 0.011$ & $0.070 \pm 0.013$ & $0.106 \pm 0.019$ & $0.077 \pm 0.031$ \\
$>45$ & $0.076 \pm 0.032$ & $0.070 \pm 0.013$ & $0.106 \pm 0.019$ & $0.077 \pm 0.031$ \\
\hline
\end{tabular}

3.13.

The fake rates obtained, (including the Monte Carlo subtraction), are listed in Table 3.9 and are plotted in Figure 3.14. Since electroweak processes become dominant with increasing muon $\mathrm{p}_{T}$, a flat fake rate for $\mathrm{p}_{T}>60 \mathrm{GeV} / c$ is assumed, using the numbers listed in the $45-60 \mathrm{GeV} / c$ column in Table 3.9.

\subsubsection{Validation of the Fake Rate Method}

The fake rate method is evaluated by testing it on Monte Carlo samples in which the true background from fakes can be determined. The fake rates are obtained from a sample of muon enriched MC QCD multijet events, and are used to estimate the fake backgrounds in Monte Carlo $t \bar{t}, W+$ jets, and QCD multijet events.

The predicted background depends on the choice of the $\mathrm{p}_{T}$ cut on the leading jet in the sample used to measure the fake rates. Figure 3.9 compares the $\mathrm{p}_{T}$ distribution of the fakeable objects in QCD, $W+$ jets, and $t \bar{t}$ events with the $\mathrm{p}_{T}$ of the fakeable objects in the independent QCD sample used to calculate the fake rates 

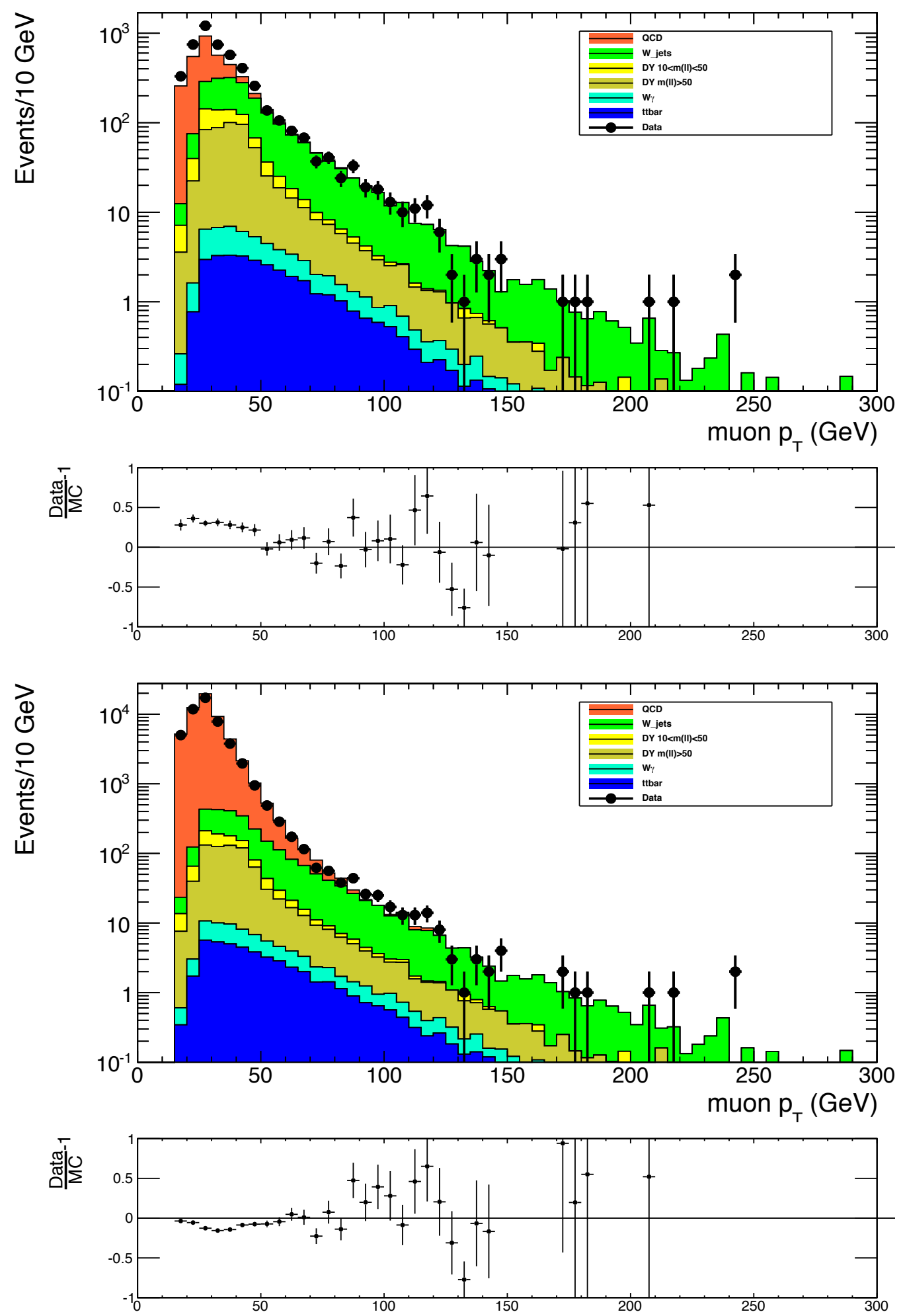

Figure 3.12: $p_{T}$ distributions for the tight muons (top) and loose muons (bottom) used in the fake rate calculation. 

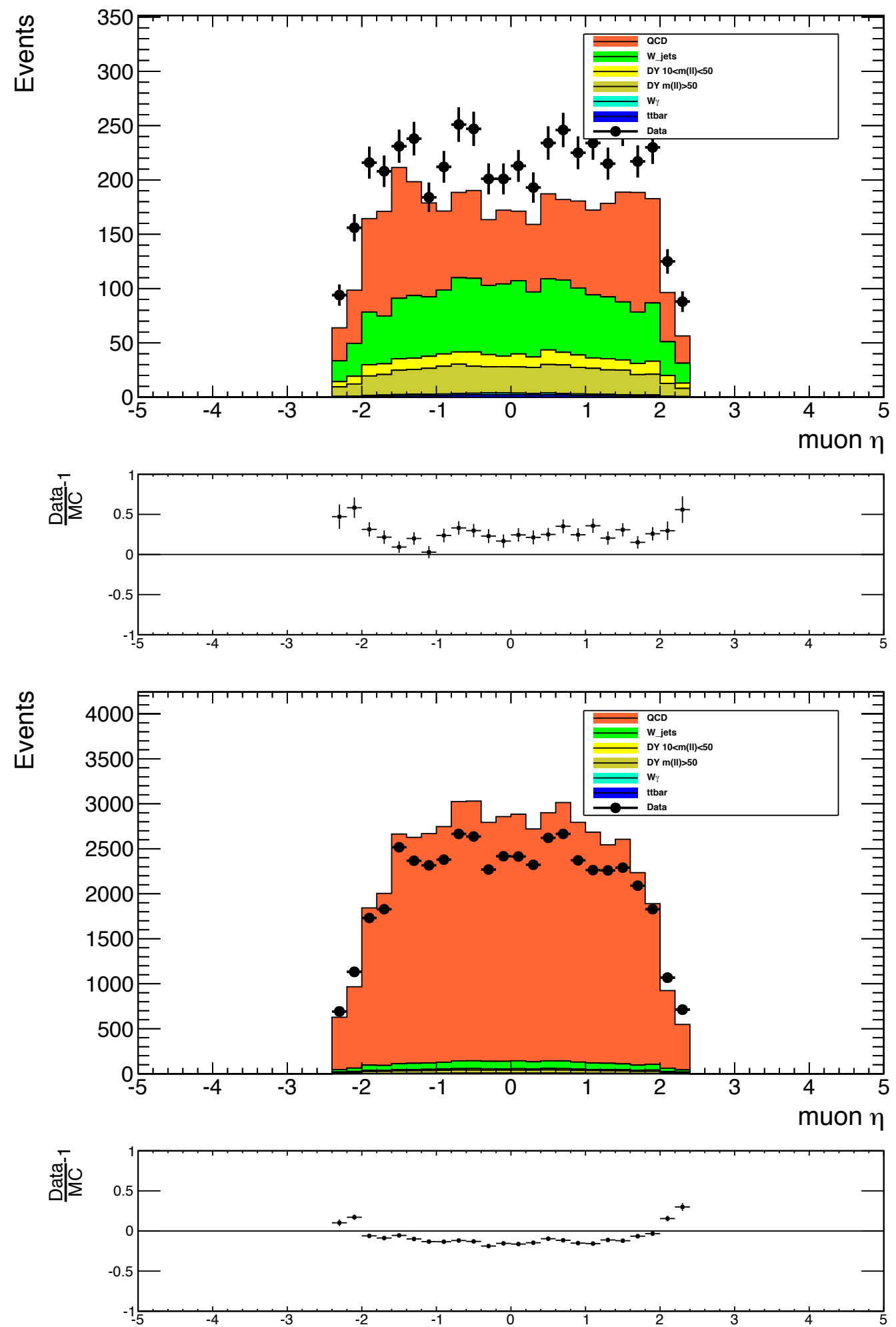

Figure 3.13: $\eta$ distributions for the tight muons (top) and loose muons (bottom) used in the fake rate calculation. 


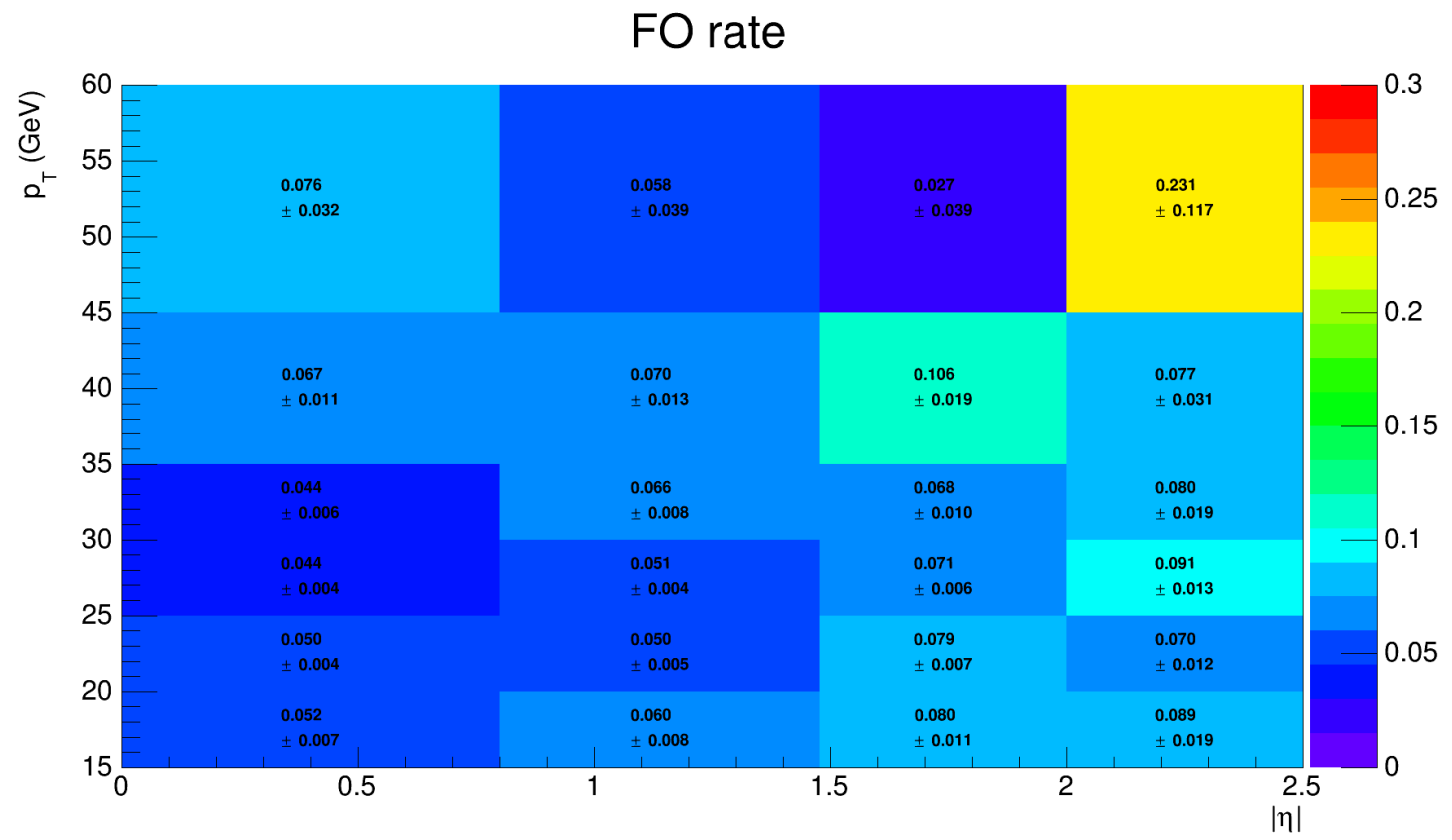

Figure 3.14: Muon fake rates obtained from data as a function of $\eta$ and $\mathrm{p}_{T}$.

with different cuts on the leading jet $\mathrm{p}_{T}$ in the latter sample. The first three samples, plotted as dots in Figure 3.9, share the analysis preselection (Section 3.4.5) but with inverted isolation requirements on the muons to pick events with loose muons and nearby jets. If two loose muons with two nearby jets are present in the event, two entries are added to the histogram. The QCD sample, plotted with different $\mathrm{p}_{T}$ cuts on the away-side jet, instead uses the same selection as used for the fake rate measurement sample to select the jet near the loose (denominator) object.

A cut of $\mathrm{p}_{T}>40 \mathrm{GeV} / c$ is used in the final calculation of the fake rate as this choice roughly matches the $\mathrm{p}_{T}$ of the fakeable objects from which the fake rates are calculated to the fakeable objects in the $t \bar{t}, W+$ jets, and QCD multijet samples. 
The $\mathrm{p}_{T}$ distribution of the jet generating the loose muon must not be misinterpreted as the $\mathrm{p}_{T}$ of the loose muon. For instance, consider a $t \bar{t}$ event in which a $b$-quark hadronizes, producing a loose muon. The $\mathrm{p}_{T}$ of the loose muon will be correlated to the initial $\mathrm{p}_{T}$ of the $b$ but it won't be the same. Furthermore, the energy of the generating particle also affects the isolation of the loose object. Matching the $\mathrm{p}_{T}$ of the generating particles in the fake rate measurement region and application region improves the level of agreement between prediction and observation in the closure tests.

Table 3.10 summarizes the results of the validation of the fake rate method for events passing the loose selection. The same selection as used to select signal events is applied, except for the MET cut. For the QCD sample the same-sign requirement is also relaxed slightly in order to increase the statistics. For $t \bar{t}$, the predicted background is overestimated by $50 \%^{15}$. The prediction for $W+$ jets is in agreement with the true fake background and is within the uncertainty of roughly $10 \%$, while for QCD the agreement is also reasonable with an uncertainty of $24 \%$.

For QCD events, an additional test of the fake rate method is performed by counting the number of tight to loose events $\left(N_{n \bar{n}}\right)$ in the MC sample and is compared with the predicted number obtained from the number of loose-loose events $\left(N_{\bar{n} \bar{n}}\right)$ scaled by the corresponding fake rate factors. The results of this test (the last row of

\footnotetext{
${ }^{15}$ The overestimate in the case of $t \bar{t}$ is also observed in the same-sign SUSY analysis $[62,63]$ and is attributed to the harder $\mathrm{p}_{T}$ distribution of fakeable objects in $t \bar{t}$ events compared to the $p_{T}$ distribution in the independent QCD sample used to calculate the fake rates. The overestimate decreases to $23 \%$ when the $\mathrm{p}_{T}$ cut is raised to $80 \mathrm{GeV} / c$.
} 
Table 3.10: Predicted (\#FR) and observed (\#MC) fake backgrounds in $t \bar{t}, W+$ jets, and QCD Monte Carlo samples for events passing preselection.

\begin{tabular}{ccccc}
\hline Sample & $p_{T}$ Cut $(\mathrm{GeV} / \mathrm{c})$ & \# FR & \# MC & $\%$ diff. \\
\hline$t \bar{t}$ & 40 & $46.3 \pm 1.5$ & 23 & $50.4 \pm 3.2$ \\
$t \bar{t}$ & 60 & $39.8 \pm 1.4$ & 23 & $42.2 \pm 3.5$ \\
$W+$ jets & 40 & $0.54 \pm 0.17$ & 1 & $84.8 \pm 30.6$ \\
QCD & 40 & $0.15 \pm 0.12$ & 0 & n.a. \\
QCD double-to-single & 40 & $7.01 \pm 0.76$ & 6 & $14.4 \pm 10.9$ \\
\hline
\end{tabular}

Table 3.10) shows that the prediction is good to within $14 \%$.

\subsection{Selection Results}

\subsubsection{Low Mass Selection Results}

The final background estimates are given in Table 3.11. The total background estimate is $11.2 \pm 1.1$ (stat) \pm 2.1 (syst) events, with the dominant contribution being the fake muon background $(8.19 \pm 0.96$ (stat) \pm 2.05 (syst) events). The data yield is in good agreement with this estimate (within errors).

After all selection cuts (described in Sections 3.5), and before the final optimization cuts, 7 events were observed in data.

The final selection cut optimization is performed on the transverse momenta of the two muons $\left(p_{T 1}\right.$ and $\left.p_{T 2}\right)$ and the invariant mass of the two muons and jets $m(\mu \mu j j)$ using the Punzi figure of merit, as described in Section 3.4.7. The results from the low mass region optimization are listed in Table 3.12. 
Table 3.11: Observed event yields and estimated backgrounds with statistical and systematic uncertainties for the low mass selection.

\begin{tabular}{ll}
\hline Source & Events \\
\hline Monte Carlo: & \\
$V V$ & $2.31 \pm 0.37($ stat $) \pm 0.35$ (syst) \\
$V V V$ & $0.12 \pm 0.03($ stat $) \pm 0.02($ syst $)$ \\
$t \bar{t} V$ & $0.12 \pm 0.05($ stat $) \pm 0.02($ syst $)$ \\
Total Monte Carlo & $2.55 \pm 0.37($ stat $) \pm 0.35($ syst $)$ \\
\hline Data-driven background estimate: & \\
Fake muon background & $8.19 \pm 0.96($ stat $) \pm 2.05($ syst $)$ \\
\hline Total background & $10.74 \pm 1.03($ stat $) \pm 2.08($ syst $)$ \\
\hline Observed in data $\left(19.7 \mathrm{fb}^{-1}\right)$ & 7 \\
\hline
\end{tabular}

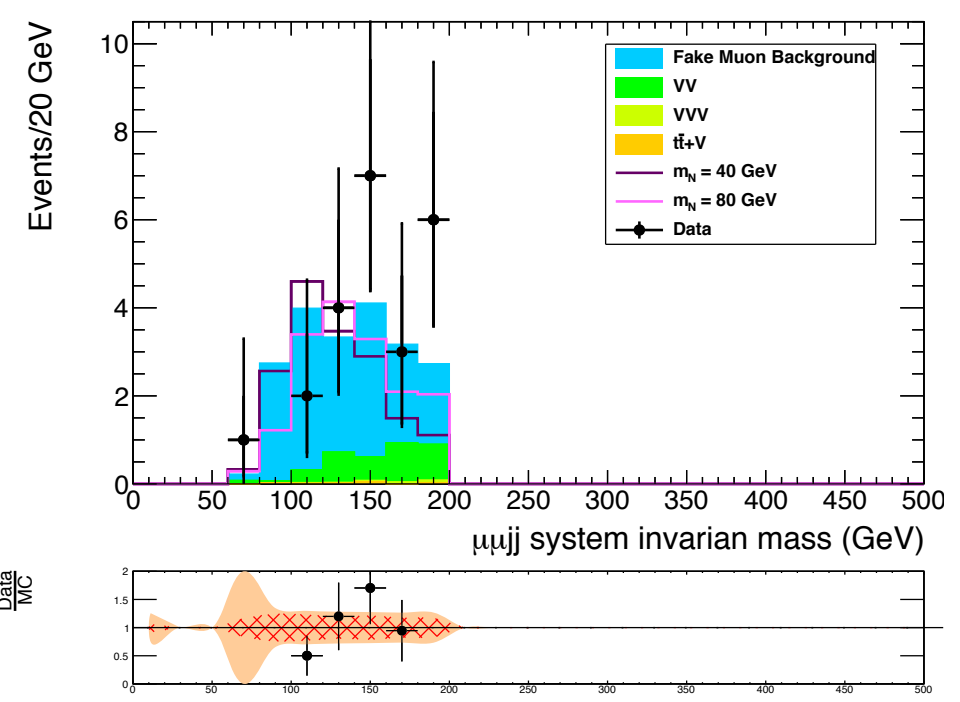

Figure 3.15: The invariant four-mass $(\mu \mu j j)$, for events passing the low mass selection. 
Table 3.12: Results of the cut optimization for the low mass Majorana neutrino region.

\begin{tabular}{ccccccc}
\hline $\begin{array}{c}N_{R} \text { Mass } \\
\left(\mathrm{GeV} / c^{2}\right)\end{array}$ & $\begin{array}{c}m(\mu \mu j j) \\
\left(\mathrm{GeV} / c^{2}\right)\end{array}$ & $\begin{array}{c}p_{T 1} \\
(\mathrm{GeV} / c)\end{array}$ & $\begin{array}{c}p_{T 2} \\
(\mathrm{GeV} / c)\end{array}$ & Tot. Bkgd. & $\begin{array}{c}\text { Eff. } \\
(\%)\end{array}$ & $N_{\text {obs }}$ \\
\hline 40 & 80 & 20 & 15 & $9.28 \pm 0.97$ & 0.78 & 7 \\
50 & 80 & 20 & 15 & $9.28 \pm 0.97$ & 0.90 & 7 \\
60 & 80 & 20 & 15 & $9.28 \pm 0.97$ & 0.73 & 7 \\
70 & 90 & 20 & 15 & $8.63 \pm 0.92$ & 0.29 & 7 \\
80 & 100 & 20 & 15 & $7.84 \pm 0.82$ & 1.21 & 7 \\
\hline
\end{tabular}

\subsubsection{High Mass Selection Results}

The final background estimates are given in Table 3.13. The total background estimate is $22.8 \pm 1.4$ (stat) \pm 3.6 (syst) events, with the dominant contribution arising from the fake muon background (13.6 \pm 1.2 (stat) \pm 3.4 (syst) events).

After the application of all selection cuts as defined in Sections 3.4.3 - 3.4.6, and before the final optimization, 19 events were observed in data.

As in the low mass region, the final selection is optimized on the $\mathrm{p}_{T}$ of the two muons, and the invariant mass of the four mass $(\mu \mu j j)$. The results of the optimization are listed in Table 3.14 .

\subsection{Systematic Uncertainties}

As this is a counting experiment the dominant sources of systematic uncertainties are those associated with our understanding of the signal model, efficiencies, and the background prediction. We take into account uncertainties from the following 
Table 3.13: Observed event yields and estimated backgrounds with statistical and systematic uncertainties for the high mass selection.

\begin{tabular}{ll}
\hline Source & Events \\
\hline Monte Carlo: & \\
$V V$ & $7.10 \pm 0.65($ stat $) \pm 1.14$ (syst) \\
$V V V$ & $0.35 \pm 0.05($ stat $) \pm 0.06($ syst $)$ \\
$t \bar{t} V$ & $0.32 \pm 0.08($ stat $) \pm 0.05$ (syst) \\
Total Monte Carlo & $9.17 \pm 0.80($ stat $) \pm 1.26($ syst $)$ \\
\hline Data-driven background estimate: & $13.62 \pm 1.16($ stat $) \pm 3.40($ syst $)$ \\
Fake muon background & $21.39 \pm 1.34($ stat $) \pm 3.59($ syst $)$ \\
\hline Total background & 19 \\
\hline Observed in data $\left(19.7 \mathrm{fb}^{-1}\right)$ &
\end{tabular}

Table 3.14: Results of the cut optimization for the 'high mass' region.

\begin{tabular}{cccccc}
\hline $\begin{array}{c}\mathrm{HMN} \\
\mathrm{GeV} / \mathrm{c}^{2}\end{array}$ & $\begin{array}{c}m(\mu \mu j \mathrm{j}) \\
\mathrm{GeV} / \mathrm{c}^{2}\end{array}$ & $\begin{array}{c}p_{T 1}, p_{T 2} \\
\mathrm{GeV} / c\end{array}$ & $\begin{array}{c}\text { Tot. Bkgd. } \\
\mathrm{GeV} / c\end{array}$ & Eff. & $N_{\text {obs }}$ \\
\hline 90 & 120 & 20,15 & $19.45 \pm 1.21$ & 1.17 & 19 \\
100 & 120 & 20,15 & $19.45 \pm 1.21$ & 4.82 & 19 \\
125 & 140 & 25,20 & $13.00 \pm 0.94$ & 11.18 & 8 \\
150 & 160 & 35,25 & $9.33 \pm 0.74$ & 13.77 & 7 \\
175 & 200 & 45,35 & $5.61 \pm 0.58$ & 13.87 & 5 \\
200 & 220 & 55,35 & $3.74 \pm 0.48$ & 15.86 & 5 \\
250 & 270 & 70,45 & $1.88 \pm 0.33$ & 16.21 & 3 \\
300 & 290 & 100,45 & $0.91 \pm 0.25$ & 14.50 & 1 \\
350 & 290 & 100,45 & $0.91 \pm 0.25$ & 15.50 & 1 \\
400 & 290 & 100,45 & $0.91 \pm 0.25$ & 15.27 & 1 \\
500 & 290 & 100,45 & $0.91 \pm 0.25$ & 12.09 & 1 \\
\hline
\end{tabular}


sources:

- Integrated Luminosity: The systematic uncertainty on the integrated luminosity is $2.6 \%$.

- Parton Distribution Functions: The ALPGEN signal Monte Carlo parton distribution function uncertainty is estimated using the method in Ref. [67]. The resulting uncertainty is $3.5 \%$ of the signal yield.

- $Q^{2}$ Scale: The $Q^{2}$ scale parameter in ALPGEN is varied from $Q^{2} / 4$ to $4 Q^{2}$. The uncertainty ranges from $4 \%$ to $10 \%$ for the low mass region, and $1 \%$ to $7 \%$ at high mass.

- Muon Trigger and Selection: The systematic uncertainty due to the uncertainty in the muon trigger efficiency is taken from the muon POG [60], with a value of $2 \%$.

- Jet Energy Scale: The jet energy is scaled by the official energy uncertainty [68] and the resulting effect on the signal efficiency is observed. The resulting systematic uncertainty is between $3 \%$ to $11 \%$ for the low mass selection, and $1 \%$ to $6 \%$ in the high mass range.

- Jet Energy Resolution: The JER depends on the Neutrino mass and is found to be $4 \%$ to $12 \%$ for the low mass region and $2 \%$ to $6 \%$ for the high mass region.

- Unclustered energy: Unclustered energy affects the signal efficiency due to the higher MET cut. The uncertainty is below $2 \%$ for the low mass region and less than $1 \%$ for the high mass region. 
Table 3.15: Summary of systematic uncertainties.

\begin{tabular}{lcccc}
\hline \multirow{2}{*}{ Majorana Neutrino Mass $\left(\mathrm{GeV} / c^{2}\right)$} \\
Source & 40 & 80 & 200 & 500 \\
\hline Integrated Luminosity & $2.60 \%$ & $2.60 \%$ & $2.60 \%$ & $2.60 \%$ \\
Muon Efficiency & $2.00 \%$ & $2.00 \%$ & $2.00 \%$ & $2.00 \%$ \\
Jet Energy Scale & $10.48 \%$ & $6.14 \%$ & $1.68 \%$ & $0.97 \%$ \\
Jet Energy Resolution & $12.05 \%$ & $7.15 \%$ & $2.94 \%$ & $2.26 \%$ \\
Unclustered energy & $0.92 \%$ & $1.71 \%$ & $0.77 \%$ & $0.78 \%$ \\
b-Tag efficiency and mistag & $0.73 \%$ & $0.75 \%$ & $0.57 \%$ & $0.49 \%$ \\
Pile Up Model & $6.91 \%$ & $7.70 \%$ & $4.09 \%$ & $3.58 \%$ \\
Parton distribution Functions & $3.50 \%$ & $3.50 \%$ & $3.50 \%$ & $3.50 \%$ \\
\hline
\end{tabular}

- b-Tag efficiency and mistag: The b-tag efficiency and mistag rate are varied independently for heavy and light flavors, the effect is less than $1 \%$.

- Pileup Model: As recommended by the Reference [69], the minimum bias crosssection was changed in order to alter the pileup profile. This results in a systematic uncertainty of $6 \%$ to $7 \%$ for the low mass region and $4 \%$ to $6 \%$ for the high mass region..

- Background Estimate: The uncertainty on the fake background is $28 \%$. The uncertainty in the normalizations of irreducible SM backgrounds are: $12 \%$ for $W Z$ [70]; $9 \%$ for $Z Z$ [71], $22 \%$ for $W W$ [71]; and $25 \%$ for the other processes, determined by varying the $Q^{2}$ scale and parton distribution functions in Monte Carlo simulations.

A summary of the systematic uncertainties is given in Table 3.15. 


\subsection{Interpretation of Results}

There is no excess of events beyond the predicted Standard Model background. Thus, limits are set on the square of the Majorana neutrino mixing element. The limits are based on the number of observed events, the number of predicted background events, and the number of predicted events from Majorana neutrino production. The 95\% confidence level limit on the cross section for heavy Majorana production is obtained using the standard CMS RooStatsCl95 package[72, 73], by applying the CLs method. See [74] for a very good review of the method.

From this, the 95\% C.L. limit on the mixing element squared can be calculated using the "bare" cross section defined as the cross section for a mixing of 1.0 obtained from the signal Monte Carlo simulation.

The limits are set on the mixing element squared, $\left|V_{\mu N}^{2}\right|$, which is directly proportional to HMN production cross section. The limits are set as a function of HMN mass, with the assumption that the mixing elements for electron and tau neutrinos are zero. Figure 3.16 shows the limits vs. HMN mass, and Figure 3.17 shows the same limits zoomed to the mass region $40-200 \mathrm{GeV} / c^{2}$.

This search is an improvement over previous searches, not only because the limits extend to higher masses than before, but because of the more advanced techniques used to set the limits. By binning the search into different regions and optimizing the selection variables with a figure of merit, a more sensitive search with tighter limits was able to be performed. Figure 3.16 includes the limits set by previous searches, as well as a line labeled preselection which shows what the observed limit would be 
without the final selection optimization as a function of HMN mass, (described in Section 3.4.7).

\subsection{Future Prospects}

With the LHC planning to start Run 2015 at a higher collision energy, with higher luminosity, the prospects for improving the exclusion at low HMN mass is not likely, due to the increased pileup, obfuscating the low $\mathrm{p}_{T}$ muons produced by a low mass HMN. However, there is already good exclusion at low mass, and the HMN mass is thought to exist most likely at a much higher mass than what is currently able to be observed. An analysis of run 2015 data will be able to set exclusions at a much higher mass range, and there is always the possibility of discovery. 


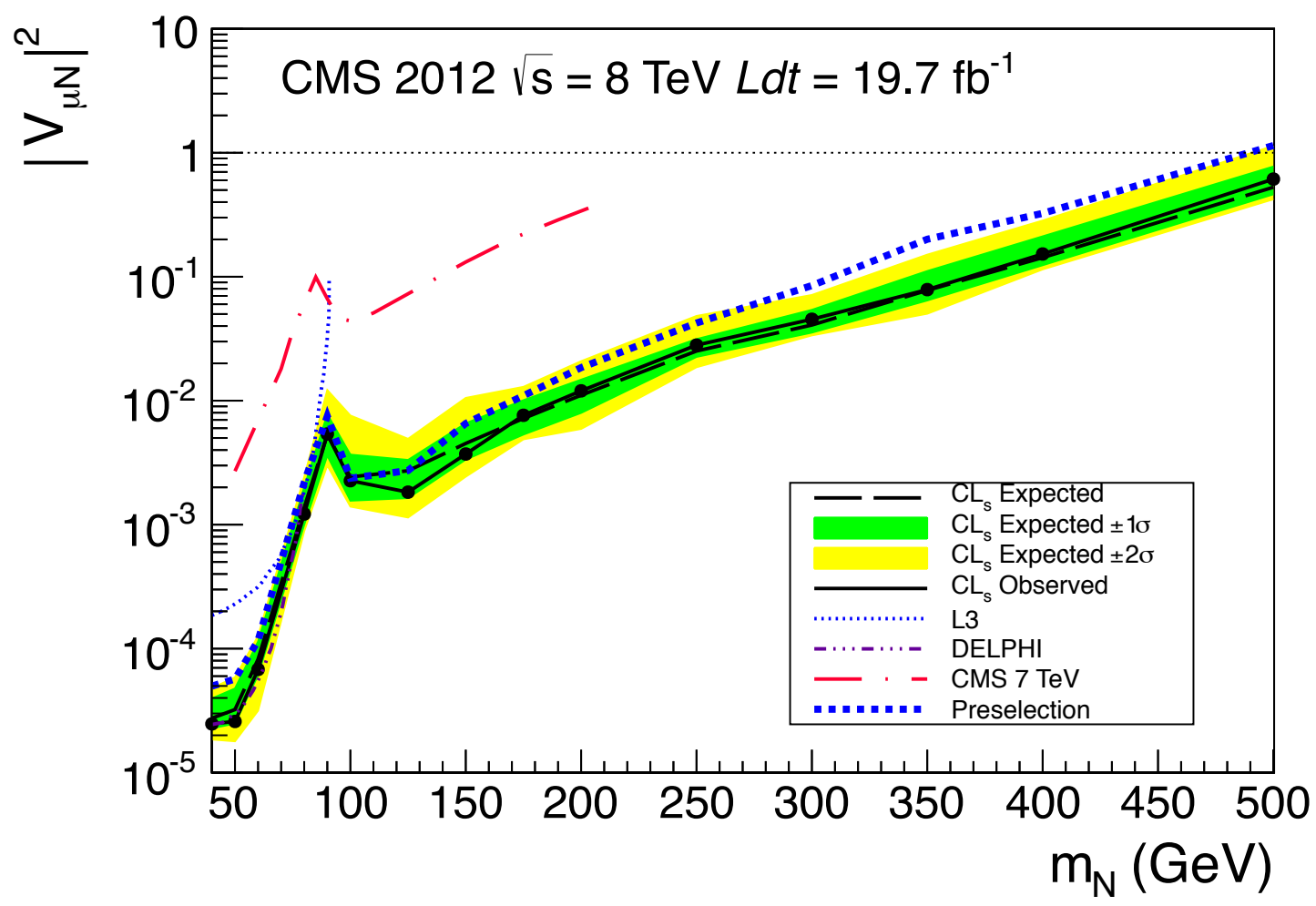

Figure 3.16: Exclusion region of the HMN mixing element vs. mass. The black hashed line is the expected limit for standard model processes, with no HMN. The black solid line shows the exclusion set by this analysis. The blue dotted line shows where the limits would be set if no selection optimization was performed on each mass point and only preselection cuts were used. The remaining lines compare previous search limits. 


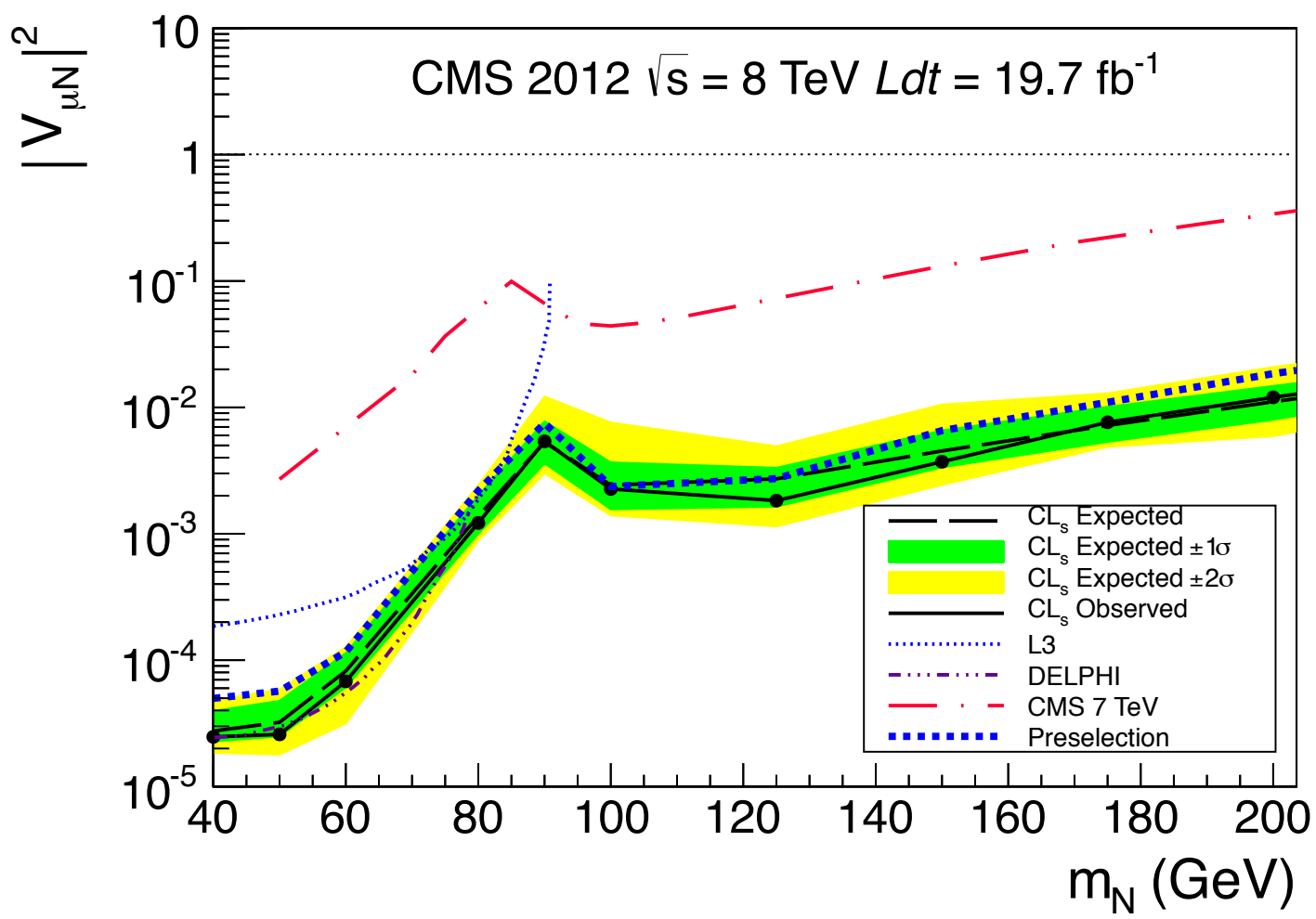

Figure 3.17: Zoom of Figure 3.16 at low mass. 


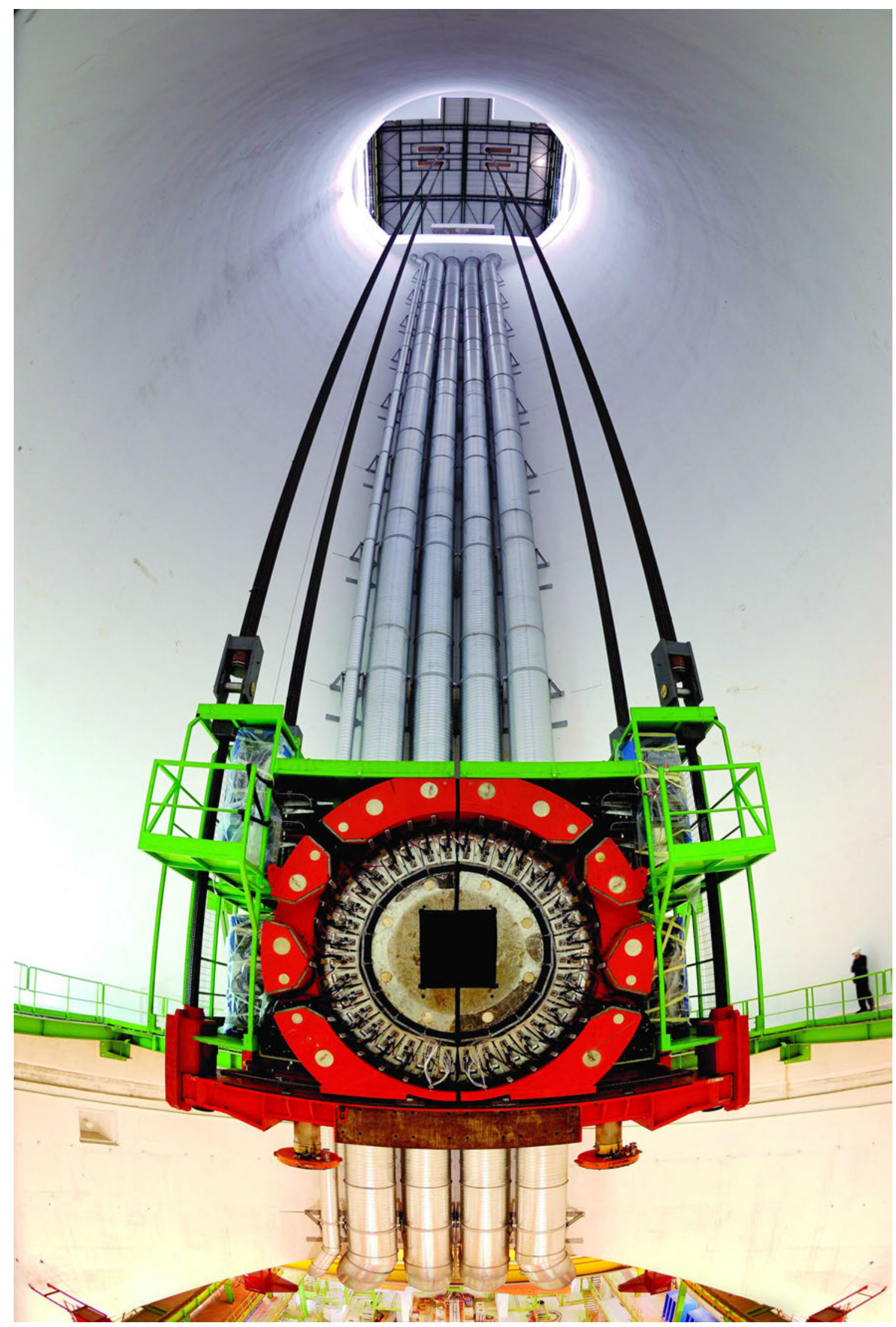

Figure 3.18: HF Lowering. 


\section{CHAPTER 4 \\ A RADIATION DAMAGE SIMULATION FOR THE HF DETECTOR}

This chapter describes a radiation damage software package developed inside CMSSW (Section 2.3.7) release 6_1_2_SLHC4_patch2, and which has subsequently been propagated to later releases.

\subsection{Introduction to $\mathrm{HF}$}

The Hadronic Forward detector is part of the HCAL system in CMS, and is designed to reconstruct jets that occur at high- $|\eta|$, close to the beam line. The HF detector is an iron absorber/quartz fiber calorimeter. Quartz fibers are embedded in large iron sheets; the iron absorber creates showers of particles, and the quartz fibers are used to generate Cherenkov radiation from the showers and transmit this light to photomultiplier tubes (PMT) in a readout box near the rear of the detector. Each iron/quartz module is referred to as a wedge, and are stacked to create a cylinder. See Figure 4.1 for a view of a row of HF wedges. Each wedge is divided into 24 towers, see Figure 4.2. The fibers in each tower are grouped together and read out by a single PMT. Towers are then clustered together in software to reconstruct jets.

As described in Section 2.4.2, good jet reconstruction relies on the quality of the clusters available for clustering, which in turn relies on the amount of light reaching the surface of the PMT from the fibers.

With the increasing energy of the LHC beam collisions, the HF detector becomes more and more relevant, as more and more interesting events occur at high- $|\eta|$. 


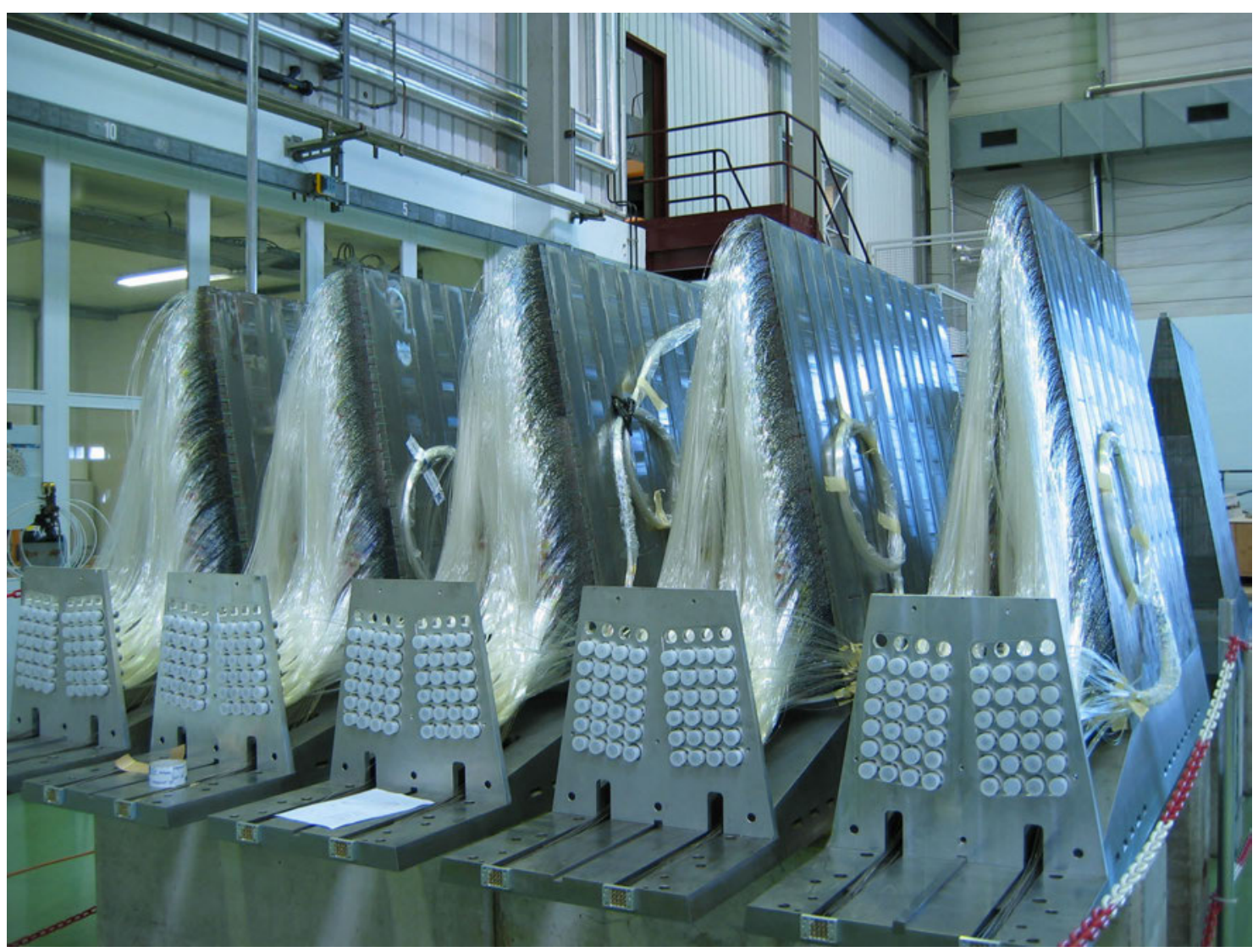

Figure 4.1: HF Wedges.

In particular, the signature for Higgs vector boson fusion production is two high- $\mid$ eta $\mid$ jets, well separated; perfect for HF.

Therefore, it is imperative that the performance of the HF detector at high integrated luminosity is understood, as its relevance will increase with energy and luminosity, but so will its radiation exposure.

In Figure 2.9 the HF detector is visible on either sides of the detector, near the beam line, labeled 'Very Forward Calorimeter'. 


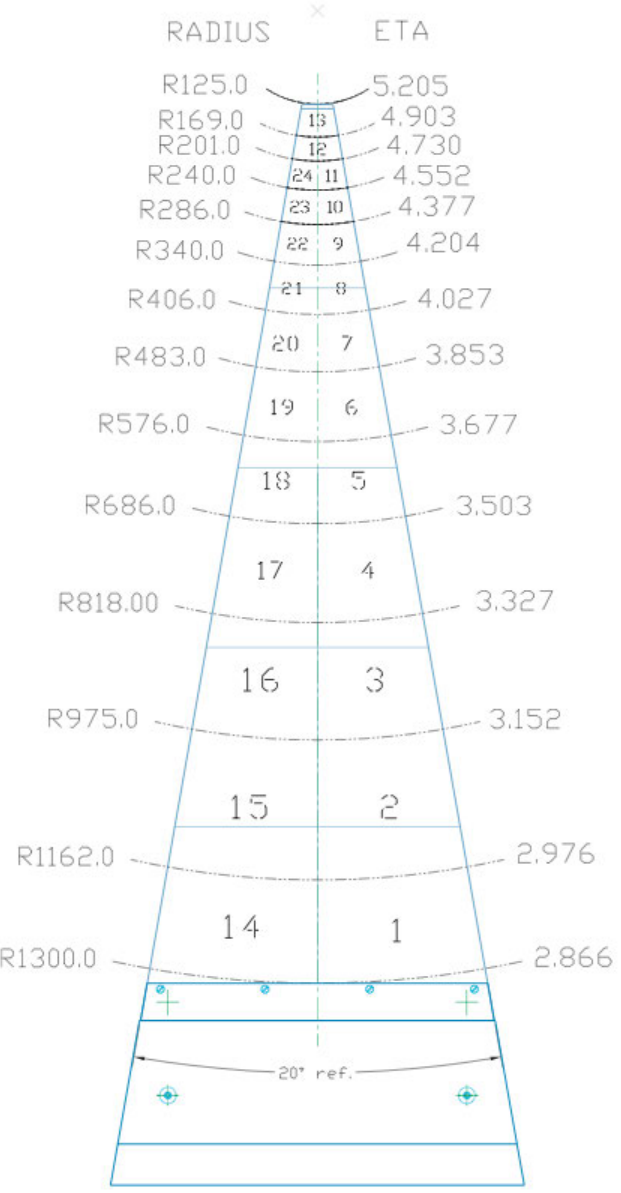

Figure 4.2: HF Tower Geometry. 


\subsection{Radiation Damage in HF}

The main source of radiation damage to $\mathrm{HF}$ will be darkening of the quartz fibers. This will impact the light transmission of the embedded fibers. The response of the HF quartz fibers to radiation has been studied in detail at the CERN PS facility IRRAD, see [75]. This work resulted in an attenuation formula as a function of delivered dose, up to 1 GRad. This formula is the crux of the HF radiation damage model:

$$
I(\lambda, D) / I(\lambda, 0)=\exp \left[(L / 4.343) \alpha(\lambda)\left(D / D_{s}\right)^{\beta(\lambda)}\right]
$$

This attenuation formula depends on parameters that can have several values, each corresponding to different experimental setups, different fibers, and different wavelengths of light. In the case of the HF fibers, $\alpha$ is taken to be 1.44 , and $\beta$ to be 0.44 . This provides the attenuation of a fiber as a function of its absorbed dose. Equation (4.1) has predicted the observed light attenuation seen by the HF radiation damage online monitoring system very well. Therefore, the radiation damage model built using this formula is expected to give realistic results.

\subsection{Modeling Radiation Damage in HF}

\subsubsection{Implementation}

The model begins by dividing the HF active area into 100 cells: 10 layers in $r$, and 10 layers in $z$. Each $z$ layer is $20 \mathrm{~cm}$, and each $r$ layer is $15.75 \mathrm{~cm}$, as shown in Fig. 4.3. Every cell is then assigned a dose variable, calculated using the online calculator linked in [76]. This dose value can be scaled according to the integrated luminosity, as 
the two quantities are linearly related. The higher the integrated luminosity passed to the simulation, the higher the doses in the cells.

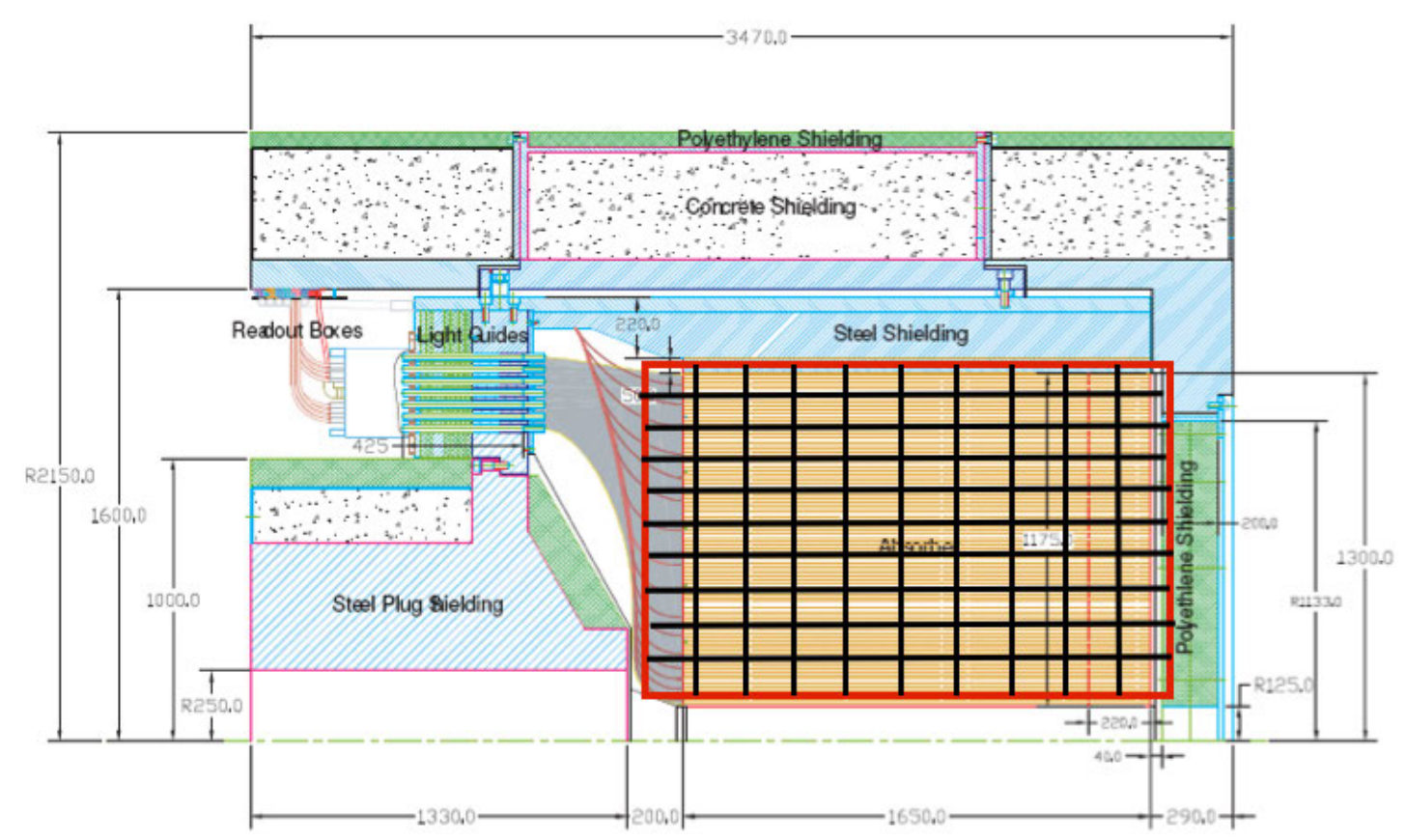

Figure 4.3: HF dose map cells.

An attenuation factor for each cell is then calculated for a given $(r, z)$ coordinate. Given the location of the PMT readouts near the rear of HF, and considering that the HF fibers travel along the $\mathrm{z}$-axis parallel to the beam line, the radiation damage model must account for the length of fiber traveled by the Cherenkov light. If it travels through a short length of fiber, it should be attenuated less, and more if it travels through a long damaged fiber. The model accounts for this by multiplying 
together the attenuation factors for each cell, starting from the cell that contains the energy deposit $\left(r_{\text {hit }}, z_{\text {hit }}\right)$, and ending with the cell at the back of HF $\left(r_{\text {hit }}, z_{0}\right)$.

The model is implemented into both Fullsim and Fastsim (see Section 2.3.8) versions of the HCAL rechit simulation package in CMSSW.

\subsection{Results}

\subsubsection{Correction Factors}

The light loss calculated by this radiation damage model can be recovered using a recalibration scheme determined from simulation. The recalibration scheme is a series of parameterizations of correction factors for each i $\eta$ and depth, as a function of integrated luminosity. The HF detector is segmented in i $\eta$ towers, and each tower has two lengths of fibers, referred to as "Depth 1" and "Depth 2", or "Long" and "Short", respectively. The parameterizations are then done for each i and each depth.

To calculate the correction factors, 2 million pions were generated over the HF $\eta$ range. For each integrated luminosity, in an interval of $500 \mathrm{fb}^{-1}$ from 0 to 10,000 $\mathrm{fb}^{-1}, 100,000$ pions were generated using the same MC seed, so only the effects of radiation damage would be considered. The correction factor is defined as:

$$
f(i \eta, d, L)=\frac{\sum_{\text {events }} E(i \eta, d, 0)}{\sum_{\text {events }} E(i \eta, d, L)}
$$

In this equation, $E$ is the sum of the energy of the simulated hits for a pion event, $d$ can be 1 (long) or 2 (short) HF fiber, and $L$ is the integrated luminosity in $\mathrm{fb}^{-1}$. A sample of the fits to the correction factors can be reviewed in Figure 4.4. As expected 
from numerous CMS radiation dose simulations, the low $\eta$ regions of HF fare the best, and the high $\eta$ regions of HF fare the worst.

\subsubsection{Jets in $\mathrm{HF}$}

To study the effect of damage to the HF fibers on calorimeter jets, 2500 down quarks were generated over the $\mathrm{HF} \eta$ range at $L=0,500$, and $3000 \mathrm{fb}^{-1}$. No pileup was included. The jets were reconstructed with and without energy recalibration enabled. The jet reconstruction efficiency was determined by matching the reconstructed calorimeter jets to the generator-level jets. Standard matching criteria were used: $\Delta R<0.3$, where $\Delta R=\sqrt{\left(\eta_{\text {Calo }}-\eta_{\text {Gen }}\right)^{2}+\left(\phi_{\text {Calo }}-\phi_{\text {Gen }}\right)^{2}}$. The results are shown in Tables 4.1 and 4.2 .

Beyond the scope of this project, and not yet fully determined is how jet energy resolution is affected by radiation damage. A brief calculation was made for $150 \mathrm{GeV}$ pions at $0 \mathrm{fb}^{-1}$ and $3000 \mathrm{fb}^{-1}$ showing a change in energy resolution of $3.4 \%$ (18.3\% to $21.7 \%$ ), which is within the normal operational range of $\mathrm{HF}$, and can be compensated for by the JERC group (JERC is described in Section 2.4.3). Figure 4.5 shows the energy resolution of HF as a function of pion energy as measured in test beam, (without any radiation damage).

\subsection{Conclusion}

A radiation damage simulation was implemented into the official CMSSW release, in both FastSim and FullSim packages. A performance study of jet reconstruction efficiency in HF was conducted using the FullSim package as a function of 

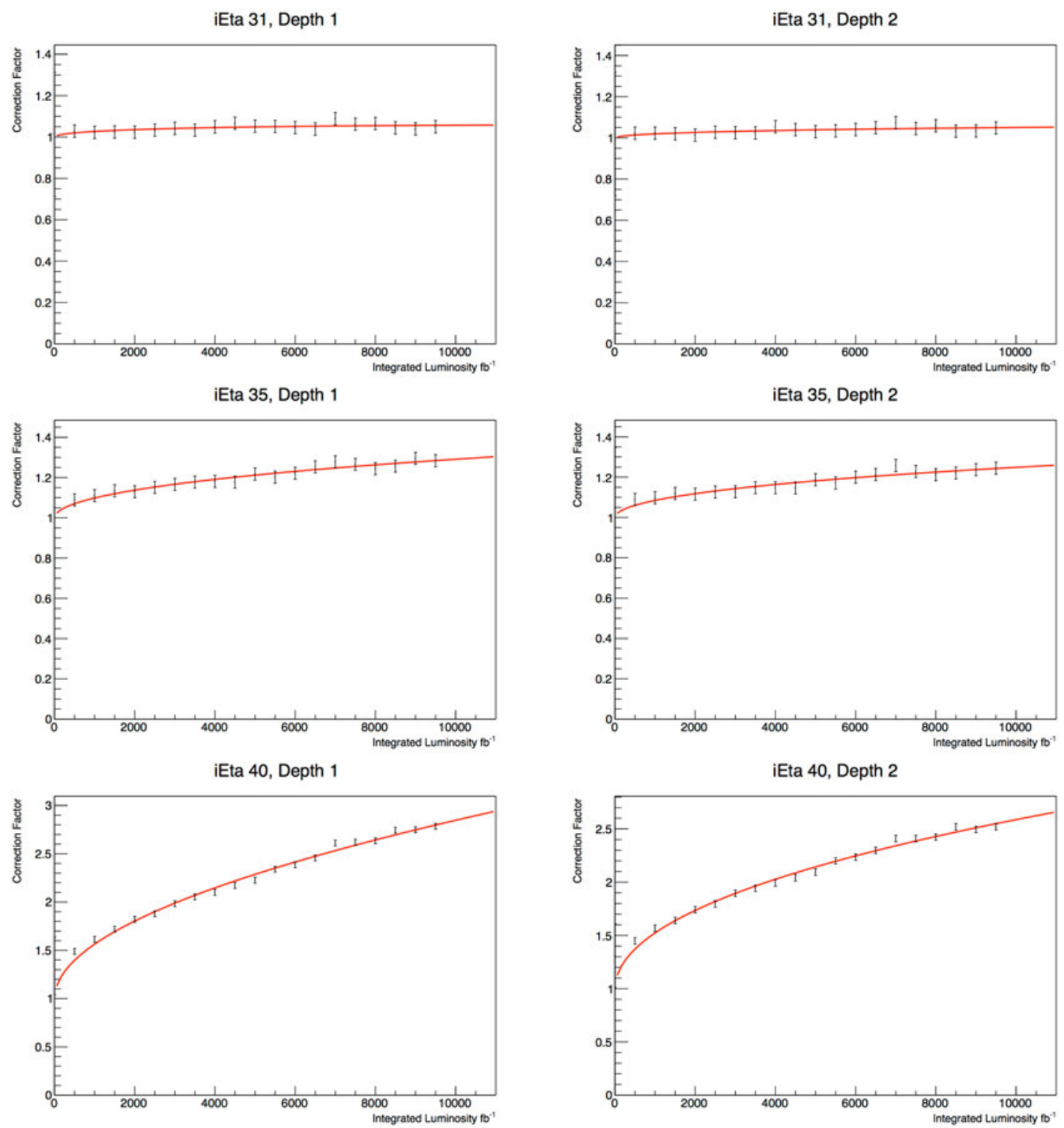

Figure 4.4: A sample of HF recalibration parameterizations. Fits are of the form $1+A \sqrt{x}+B x$. 
Table 4.1: Efficiencies for varying jet $\mathrm{p}_{T}$ and integrated luminosity for jets from single down quarks in the HF.

\begin{tabular}{|c|c|}
\hline $\mathrm{fb}^{-1}$ & Efficiency (\# Calo Jets / \# Gen Jets) \\
\hline \multicolumn{2}{|c|}{50 GeV Jets With Recalibration } \\
\hline 0 & $89.0 \%$ \\
\hline 500 & $86.5 \%$ \\
\hline 3000 & $84.0 \%$ \\
\hline \multicolumn{2}{|c|}{$100 \mathrm{GeV}$ Jets No Recalibration } \\
\hline 0 & $97.4 \%$ \\
\hline 500 & $97.2 \%$ \\
\hline 3000 & $95.9 \%$ \\
\hline \multicolumn{2}{|c|}{$100 \mathrm{GeV}$ Jets With Recalibration } \\
\hline 0 & $97.4 \%$ \\
\hline 500 & $97.2 \%$ \\
\hline 3000 & $96.3 \%$ \\
\hline \multicolumn{2}{|c|}{150 GeV Jets With Recalibration } \\
\hline 0 & $98.2 \%$ \\
\hline 500 & $98.5 \%$ \\
\hline 3000 & $97.8 \%$ \\
\hline
\end{tabular}

Note: Statistical errors are negligible and omitted for brevity. 
Table 4.2: $100 \mathrm{GeV}$ jet efficiency vs. $\eta$.

\begin{tabular}{|c|c|c|c|c|}
\hline \multirow[t]{2}{*}{$\mathrm{fb}^{-1}$} & \multicolumn{4}{|c|}{$|\eta|$ Region } \\
\hline & $3-3.5$ & $3.5-4$ & $4-4.5$ & $4.5-5$ \\
\hline \multicolumn{5}{|c|}{ No Recalibration } \\
\hline 0 & $100 \%$ & $99.3 \%$ & $98.9 \%$ & $91.1 \%$ \\
\hline 500 & $99.2 \%$ & $99.6 \%$ & $99.0 \%$ & $90.0 \%$ \\
\hline 3000 & $99.3 \%$ & $98.6 \%$ & $97.8 \%$ & $87.8 \%$ \\
\hline \multicolumn{5}{|c|}{ With Recalibration } \\
\hline 500 & $99.8 \%$ & $99.8 \%$ & $99.1 \%$ & $90.5 \%$ \\
\hline 3000 & $98.6 \%$ & $99.4 \%$ & $99.5 \%$ & $88.4 \%$ \\
\hline
\end{tabular}

Note: Statistical errors are negligible and omitted for brevity.

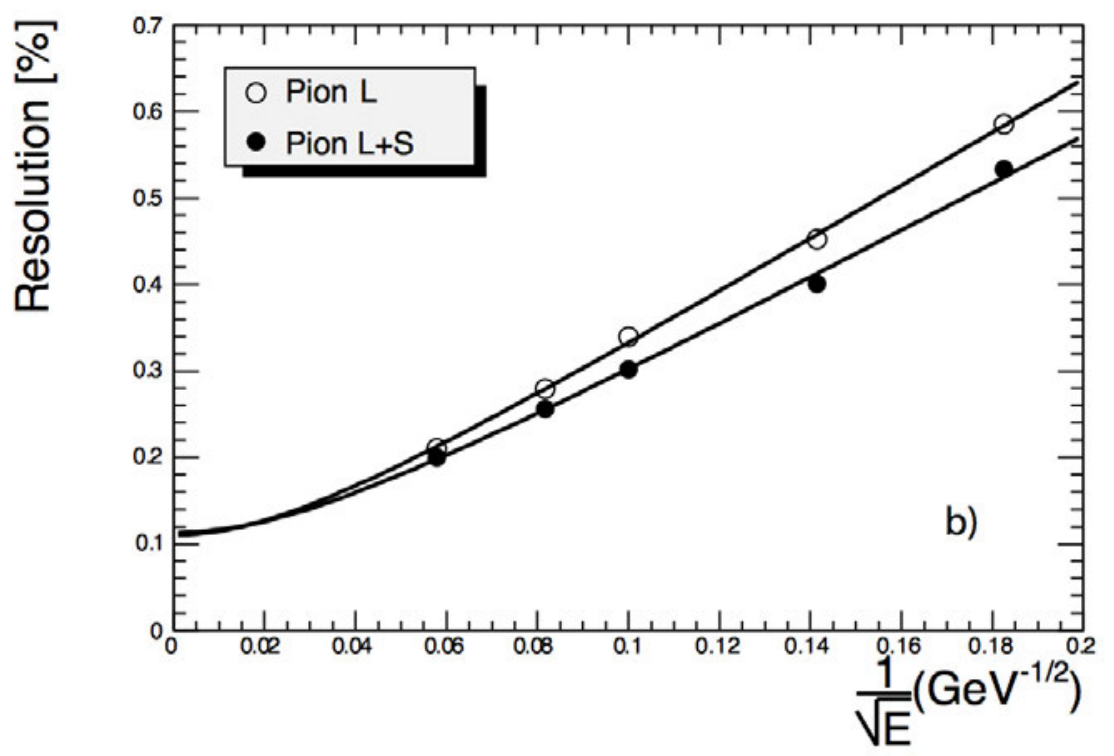

Figure 4.5: Energy resolution of the HF detector. [77] 
integrated luminosity. The simulation shows that HF will lose some efficiency, however, this loss can be compensated with calibration factors. The calibration factors were calculated for each tower and depth in HF, and implemented back into the software package with a boolean for easily enabling or disabling both the damage and the calibration.

The outlook is good for HF jet reconstruction efficiency through the entire lifetime of the CMS detector.

\subsection{Future prospects}

An updated radiation dose map has been created using the FLUKA package. This new map has better granularity in HF which could lend itself to producing a more accurate simulation. A new simulation will be implemented using new correction factors calculated with the FLUKA dose map, and results will be compared with the results of this simulation.

Beyond that, a full suite of studies can now be performed using this package by anyone in the CMS collaboration, ensuring robust planning for operating HF well into the future. 


\section{REFERENCES}

[1] D. Griffiths. Introduction to Elementary Particles. Wiley VCH, 2008.

[2] G.D. Coughlan and J.E. Dodd. The ideas of particle physics. Cambridge University Press, 1991.

[3] F. Halzen and A. D. Martin. Quarks and Leptons: An Introductory Course in Modern Particle Physics. John Wiley \& Sons, 1984.

[4] F. Reines and C. L. Jr. Cowan. Detection of the free neutrino. Physical Review, 92:830, 1953.

[5] The Reines-Cowan experiments, detecting the poltergeist. Los Alamos Science, (25), 1997.

[6] E. J. Konopinski and H. M. Mahmoud. The universal Fermi interaction. Physical Review, 92(4):1045, 1953.

[7] L. M. Lederman et al. Observation of high-energy neutrino reactions and the existence of two kinds of neutrinos. Physical Review Letters, 9(1):36, 1962.

[8] M. Gell-Mann and Y. Ne'Eman. The Eightfold Way. W. A. Benjamin, 1964.

[9] M. Gell-Mann. The eightfold way: A theory of strong interaction symmetry. 1961.

[10] J. Elias-Miro et al. Higgs mass implications on the stability of the electroweak vacuum. 2011.

[11] L. Di Luzio and L. Mihaila. On the gauge dependence of the standard model vacuum instability scale. Journal of High Energy Physics, 2014(6), 2014.

[12] CDF Collaboration. Evidence for a narrow near-threshold structure in the $\mathrm{J} / \psi \phi$ mass spectrum in $\mathrm{B}+\rightarrow \mathrm{J} / \psi \phi \mathrm{K}+$ decays. Phys. Rev. Lett., 102:242002, Jun 2009.

[13] Observation of a peaking structure in the mass spectrum from decays. Physics Letters B, 734(0):261 - 281, 2014.

[14] LHCb Collaboration. Observation of the resonant character of the $z(4430)^{-}$ state. Phys. Rev. Lett., 112:222002, Jun 2014. 
[15] ILC. The international linear collider. http://www.linearcollider.org/ILC [Accessed:2014-7-2].

[16] L. Evans and P. Bryant. LHC machine. Journal of Instrumentation, 3(08):S08001, 2008.

[17] S Baird. Accelerators for pedestrians; rev. version. Technical Report AB-Note2007-014. CERN-AB-Note-2007-014. PS-OP-Note-95-17-Rev-2. CERN-PS-OPNote-95-17-Rev-2, CERN, Geneva, Feb 2007.

[18] T. Berners-Lee et al. World-Wide Web: The Information Universe. Internet Research, 2:52-58, 1992.

[19] F.J. Hasert et al. Observation of neutrino-like interactions without muon or electron in the gargamelle neutrino experiment. Physics Letters B, 46(1):138140, 1973.

[20] G. Arnison et al. Experimental observation of isolated large transverse energy electrons with associated missing energy at $\mathrm{s}=540$ gev. Physics Letters $B, 122(1): 103-116,1983$.

[21] M. Banner et al. Observation of single isolated electrons of high transverse momentum in events with missing transverse energy at the $\{\mathrm{CERN}\}$ pp collider. Physics Letters B, 122(56):476 - 485, 1983.

[22] R.J. Steinhagen. LHC beam stability and feedback control - orbit and energy.

[23] CMS Collaboration. CMS Physics: Technical Design Report Volume 1: Detector Performance and Software. 2006. There is an error on cover due to a technical problem for some items.

[24] The CMS hadron calorimeter project: Technical Design Report. Technical Design Report CMS. CERN, Geneva, 1997.

[25] CMSSW. Github repository. https://github.com/cms-sw/cmssw [Accessed:2014-7-3].

[26] R. Fruhwirth. Application of Kalman filtering to track and vertex fitting. Nucl.Instrum.Meth., A262:444-450, 1987.

[27] H. on behalf of the CMS collaboration) Kirschenmann. Determination of the jet energy scale in cms. Journal of Physics: Conference Series, 404(1):012013, 2012.

[28] https://twiki.cern.ch/twiki/bin/viewauth/CMS/JetEnergyScale. 
[29] S. Chatrchyan et al. Identification of b-quark jets with the CMS experiment. JINST, 8:P04013, 2013.

[30] R.N. Mohapatra and P.B. Pal. Massive Neutrinos in Physics and Astrophysics. World Scientific Publishing Company, 2004.

[31] M. Fukugita and T. Yanagida. Physics of neutrinos and applications to astrophysics. Texts and monographs in physics. Springer, 2003.

[32] V. Barger, D. Marfatia, and K. Whisnant. Progress in the physics of massive neutrinos. Int. J. Mod. Phys. E, 12:569-647, 2003.

[33] R. M. Bionta, G. Blewitt, C. B. Bratton, D. Casper, and A. Ciocio. Observation of a neutrino burst in coincidence with supernova 1987A in the Large Magellanic Cloud. Physical Review Letters, 58:1494-1496, April 1987.

[34] R.N. Mohapatra and A.Y. Smirnov. Neutrino mass and new physics. Annual Review of Nuclear and Particle Science, 56:569-628, 2006.

[35] P. Minkowski. $\mu \rightarrow e \gamma$ at a Rate of One Out of 1-Billion Muon Decays? Phys.Lett., B67:421, 1977.

[36] M. Gell-Mann, P. Ramond, and R. Slansky. In P.V. Nieuwenhuizen and D.Z. Freedman, editors, Supergravity: proceedings of the Supergravity Workshop at Stony Brook, page 315. North-Holland, 1979.

[37] T. Yanagida. In O. Sawada and A. Sugamoto, editors, Proceedings of the Workshop on the Unified Theory and the Baryon Number in the Universe, page 95. National Labratory for High Energy Physics (KEK), 1979.

[38] R. N. Mohapatra and G. Senjanovic. Neutrino Mass and Spontaneous Parity Violation. Phys.Rev.Lett., 44:912, 1980.

[39] W. Keung and G. Senjanovic. Majorana Neutrinos And The Production Of The Right-Handed Charged Gauge Boson. Phys.Rev.Lett., 50:1427, 1983.

[40] D. A. Dicus, D. D. Karatas, and P. Roy. Lepton nonconservation at supercollider energies. Phys.Rev., D44:2033-2037, 1991.

[41] A. Datta, M. Guchait, and A. Pilaftsis. Probing lepton number violation via majorana neutrinos at hadron supercolliders. Phys.Rev., D50:3195-3203, 1994.

[42] F.M.L. Almeida et al. On a signature for heavy Majorana neutrinos in hadronic collisions. Phys.Rev., D62:075004, 2000. Latex2e(epsfig), 12 pages, 8 figures, to appear Physical Review D. 
[43] O. Panella, M. Cannoni, C. Carimalo, and Y.N. Srivastava. Signals of heavy Majorana neutrinos at hadron colliders. Phys.Rev., D65:035005, 2002.

[44] T. Han and B. Zhang. Signatures for Majorana neutrinos at hadron colliders. Phys.Rev.Lett., 97:171804, 2006.

[45] F. del Aguila, J.A. Aguilar-Saavedra, and R. Pittau. Heavy neutrino signals at large hadron colliders. JHEP, 0710:047, 2007.

[46] A. Atre et al. The Search for Heavy Majorana Neutrinos. JHEP, 0905:030, 2009.

[47] P. Abreu et al. Search for neutral heavy leptons produced in Z decays. Z.Phys., C74:57-71, 1997.

[48] O. Adriani et al. Search for isosinglet neutral heavy leptons in Z0 decays. Phys.Lett., B295:371-382, 1992.

[49] S. Chatrchyan et al. Search for heavy Majorana neutrinos in $\mu^{+} \mu^{+}\left[\mu^{-} \mu^{-}\right]$and $e^{+} e^{+}\left[e^{-} e^{-}\right]$events in $p p$ collisions at $\sqrt{s}=7 \mathrm{TeV}$. Phys. Lett. B, 717:109-128, 2012.

[50] The ATLAS Collaboration. Search for majorana neutrino production in pp collisions at $\operatorname{sqrt}(\mathrm{s})=7$ tev in same-sign dimuon final states with the atlas detector. ATLAS Conference Note, ATLAS-CONF-2012-139, 2012.

[51] F. del Aguila, J.A. Aguilar-Saavedra, and R. Pittau. Heavy neutrino signals at large hadron colliders. JHEP, 0710:047, 2007.

[52] M. L. Mangano et al. ALPGEN, a generator for hard multiparton processes in hadronic collisions. JHEP, 07:001, 2003.

[53] M.R. Wahlley, D. Bourilkov, and R.C. Group. The les houches accord PDFs (LHAPDF) and LHAGLUE. 2005.

[54] T. Sjostrand, S. Mrenna, and P. Z. Skands. PYTHIA 6.4 physics and manual. JHEP, 0605:026, 2006.

[55] R. Gavin et al. FEWZ 2.0: A code for hadronic Z production at next-to-nextto-leading order. Comput. Phys. Commun., 182:2388-2403, 2011.

[56] R. Gavin et al. W Physics at the LHC with FEWZ 2.1. Comput. Phys. Commun., 184:208-214, 2013.

[57] D. Kim, Y. Oh, Y. Yang, and J. Lee. Pdf uncertainties and k-factor for the $w^{\prime}$ search at 8 tev collisions. CMS Note, AN-2012-172, 2012. 
[58] S. Agostinelli, J. Allison, K. Amako, J. Apostolakis, H. Araujo, et al. GEANT4: A Simulation toolkit. Nucl.Instrum.Meth., A506:250-303, 2003.

[59] G. Punzi. Sensitivity of searches for new signals and its optimization. arXiv, 0308063, 2003.

[60] Piet Verwilligen's talk at the muon POG. https://indico.cern.ch/event/ 289302/material/slides/0? contribId=2.

[61] W. Andrews et al. Search for Higgs Boson decay to two W bosons in the fully leptonic final state at $\sqrt{7} \mathrm{TeV}$ with 2011 data with the CMS detector. CMS Note, AN-2011-147, 2011.

[62] D. Barge et al. Inclusive search for new physics with same-sign dileptons using early lhc data. CMS Note, AN-2010-247, 2010.

[63] D. Barge et al. Search for new physics with same-sign dileptons using the 2011 dataset of cms. CMS Note, AN-2011-258, 2011.

[64] G. Bauer, J. Bendavid, E. Butz, et al. Lepton effiencies for the inclusive w cross section measurement with 36.1pb ${ }^{-1}$. CMS Note, AN-2011-097, 2011.

[65] H. Bakhshian et al. Computing the contamination from fakes in leptonic final states. CMS Note, AN-2010-261, 2010.

[66] S. Halil and C. G. Tully. Search for third generation scalar leptoquarks decaying to top quark - tau lepton pairs in pp collisions. CMS Note, AN-2012-289, 2012.

[67] D. Bourilkov, R. C. Group, and M. R. Whalley. LHAPDF: PDF use from the Tevatron to the LHC. 2006.

[68] J. Berger, D. del Re, R. Eusebi, T. Hauth, I. Iashvili, S. Jain, M. Kaya, O. Kaya, K. Mishra, F. Pandolfi, D. Piparo, G. Quast, P. Schieferdecker, S. Sengupta, and M. Voutilainen. Absolute jet energy correction and its uncertainty with $36 p b^{-1}$. CMS Note, AN-2011/105, 2011.

[69] https://twiki.cern.ch/twiki/bin/view/CMS/PileupSystematicErrors.

[70] CMS Collaboration. Measurement of wz production rate. CMS Physics Analysis Summary, CMS-PAS-SMP-12-006, 2013.

[71] S. Chatrchyan et al. Measurement of $\mathrm{W}+\mathrm{W}$ - and ZZ production cross sections in pp collisions at $\operatorname{sqrt}(\mathrm{s})=8 \mathrm{TeV}$. Phys. Lett. B, 721:190-211, 2013. 
[72] L. Moneta, K. Belasco, K. S. Cranmer, S. Kreiss, A. Lazzaro, et al. The RooStats Project. PoS, ACAT2010:057, 2010.

[73] https://twiki.cern.ch/twiki/bin/viewauth/CMS/RooStatsCl95.

[74] A. L. Read. http://cds.cern.ch/record/451614/files/open-2000-205. pdf.

[75] K. Cankoçak et al. Radiation-hardness measurements of high oh content quartz fibres irradiated with 24 gev protons up to 1.25 grad. Nuclear Instruments and Methods in Physics Research Section A, 585:20-27, 2007.

[76] P.C. Bhat, A.P. Singh, and N.V. Mokhov. Mars simulation for pp at 14 tev, 2008. http://cmstrk.fnal.gov/radsim/LHC_DoseFlux_Calculator.html.

[77] G Baiatian et al. Design, performance and calibration of the cms forward calorimeter wedges. Technical Report CMS NOTE 2006 044, CERN, Geneva, Feb 2006. 